\title{
IDENTIFICATION OF NRF1 (NFE2L1) AS A HYPOXIA- INDUCIBLE FACTOR AND ITS OXYGEN-DEPENDENT REGULATION IN VITRO AND IN VIVO
}

\author{
by
}

\section{NIKOLAI L. CHEPELEV}

B. Sc. H. Carleton University, 2006

A thesis submitted to the Faculty of Graduate and Postdoctoral Affairs in partial fulfillment of the requirements for the degree of

Doctor of Philosophy

in

Biology

Department of Biology

Ottawa-Carleton Institute of Biology

Carleton University

Ottawa, Ontario, Canada

September 2011

(C) 2011 Nikolai Chepelev 
Library and Archives

Canada

Published Heritage

Branch

395 Wellington Street Ottawa ON K1A ON4 Canada
Bibliothèque et

Archives Canada

Direction du

Patrimoine de l'édition

395 , rue Wellington

Ottawa ON K1A ON4

Canada

Your file Votre référence

ISBN: 978-0-494-83249-3

Our file Notre référence

ISBN: 978-0-494-83249-3

NOTICE:

AVIS:

The author has granted a nonexclusive license allowing Library and Archives Canada to reproduce, publish, archive, preserve, conserve, communicate to the public by telecommunication or on the Internet, loan, distribute and sell theses worldwide, for commercial or noncommercial purposes, in microform, paper, electronic and/or any other formats.

The author retains copyright ownership and moral rights in this thesis. Neither the thesis nor substantial extracts from it may be printed or otherwise reproduced without the author's permission.

L'auteur a accordé une licence non exclusive permettant à la Bibliothèque et Archives Canada de reproduire, publier, archiver, sauvegarder, conserver, transmettre au public par télécommunication ou par l'Internet, prêter, distribuer et vendre des thèses partout dans le monde, à des fins commerciales ou autres, sur support microforme, papier, électronique et/ou autres formats.

L'auteur conserve la propriété du droit d'auteur et des droits moraux qui protège cette thèse. $\mathrm{Ni}$ la thèse ni des extraits substantiels de celle-ci ne doivent être imprimés ou autrement reproduits sans son autorisation.

In compliance with the Canadian Privacy Act some supporting forms may have been removed from this thesis.

While these forms may be included in the document page count, their removal does not represent any loss of content from the thesis.
Conformément à la loi canadienne sur la protection de la vie privée, quelques formulaires secondaires ont été enlevés de cette thèse.

Bien que ces formulaires aient inclus dans la pagination, il n'y aura aucun contenu manquant.

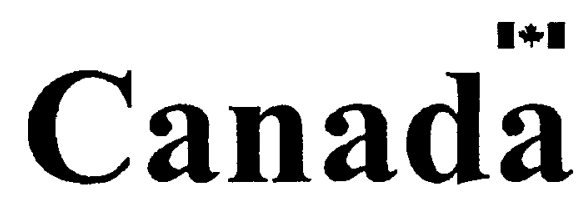




\section{Abstract}

Oxygen and iron-sensing pathways are tightly interrelated. For example, a group of proteins known as hypoxia-inducible factors (HIFs) mediate the responses of mammalian cells to variations in ambient oxygen levels and are regulated in an iron- and oxygendependent manner. HIFs activate the expression of genes responsible for iron metabolism, transport and storage. Similarly, both iron overload and oxygen deprivation (hypoxia) conditions can stimulate the production of reactive oxygen species (ROS) that can be harmful to biomacromolecules, altering their structure and function. The cap-ncollar (CNC) subfamily of basic leucine zipper (bZIP) transcriptional factors includes Nrf (nuclear factor-erythroid $2 \mathrm{p} 45$ subunit-related factor) 1 and 2 proteins that activate the expression of the genes, important to iron storage and metabolism, as well as to the cellular defense against ROS. The CNC-bZIP factors bind to a DNA sequence known as the Antioxidant-Response Element (ARE) to either activate or repress the ARE-driven gene expression under various physiological conditions. This project revealed that hypoxic conditions activate Nrfl, demonstrating further interrelatedness between the oxygen and iron metabolism pathways. In addition to describing oxygen-dependent regulation of Nrfl, it has been demonstrated that bisphenol A (BPA), a compound thought to produce ROS intracellularly and a common environmental pollutant, can also activate Nrfl and the expression of its target genes. Finally, using mouse models, it was shown that the protein binding to an ARE sequence diminishes with age in the lung with concomitant changes in the Nrf1/Nrf2 ratio. Taken together, the work presented in this thesis reveals how Nrf1 is regulated under different, oxygen-dependent conditions. Since 
$\mathrm{Nrfl}$ can be very important for human health and Nrfl is a key regulator of the cellular antioxidant defense system and proteasomal gene expression, this work can be a useful starting point for developing the strategies for pharmacological activation of $\mathrm{Nrfl}$, which may be beneficial in numerous diseases where oxidative stress is a factor, such as neurodegenerative diseases, brain injury, acute lung injury and cerebral ischemia. 


\section{Preface}

This thesis is written in an integrated thesis format, summarizing the work that, at the time of the thesis submission, has been published already (Chapter 1), submitted (Chapters 3 and 4) or will soon be submitted for a publication in a peerreview journal (Chapter 5).

Status of the manuscripts at the time of thesis submission

Chapter 1:

Chepelev, N.L., and Willmore, W.G. 2011. Regulation of iron pathways in response to hypoxia. Free Radic. Biol. Med. 50, 645-666.

Chapter 3:

Chepelev, N.L., Bennitz, J, Huang, T., McBride, S., and Willmore, W.G. 2011. The Nrf1 CNC-bZIP protein is regulated by the proteasome and activated by hypoxia. Submitted to PLoS ONE on July 20, 2011; submission number: PONE-S-11-17302. The manuscript is currently undergoing the revisions suggested by the reviewers.

\section{Chapter 4:}

Chepelev, N.L., Enikanolaiye, M., Chepelev, L.L., Chen, Q., Scoggan, K., Coughlan, M., Jin, X., and Willmore, W.G. 2011. Bisphenol A activates Nrf1/2-antioxidant response element pathway in HEK293A cells. Submitted to Chemical Research in Toxicology on 
March 31, 2011; submission number: tx-2011-00132j. The manuscript is currently undergoing the revisions suggested by the reviewers.

\section{Chapter 5:}

Chepelev, N.L., Zhang, H., Liu, H., McBride, S., Willmore, W.G., Morgan, T., Finch, C., Davies, K., and Forman, H. The Yin-yang of Nrf1 and Nrf2 in aging mouse lung. The expected date of submission is August 15, 2011.

\section{Statement of contributions}

I have contributed towards the research described in this thesis as follows:

1. Jointly with Dr. Willmore, I formulated the hypotheses,

2. I planned and executed the experiments and collected the data,

3. I analyzed the data, jointly with Dr. Willmore,

4. In collaboration with Dr. Willmore, I trained and co-supervised six undergraduate students: Joshua Bennitz, who helped with the optimization of luciferase assays and the cloning of N-terminally-tagged Nrfl, Ioana Nicolau and Julia DiLabio, who participated in some preliminary data collection, Ting Huang and Skye McBride, who participated in C-terminally-tagged Nrf1-FLAG cloning and site-directed mutagenesis and Mutiat Enikanolaiye, who participated in the glutathione and ROS assays and antibody microarrays,

5. With the help of Dr. Willmore, I prepared the manuscripts that form the basis for this thesis, 
6. In collaboration with the Health Canada group (Dr. Xiaolei (Dawn) Jin) and Dr. Willmore, I designed and performed the experiments with BPA described in Chapter 4 ,

7. In collaboration with the University of Southern California, Los Angeles (USC LA) group (Dr. Henry Forman (PI), Dr. Hongqiao Zhang, Dr. Kelvin Davies (PI) and Dr. Caleb Finch (PI)) and Dr. Willmore, who oversaw my proposal for the SFRBM Mini-Fellowship application, I participated in the experimental design of the experiments described in Chapter 5. Together with Dr. H. Zhang, an assistant professor working in Dr. Forman's lab, I was involved in data collection and analyses using mouse lung tissue and drafted a manuscript as described in Chapter 5 .

I would like to formally acknowledge the contributions of my co-authors of the manuscripts that form the basis of this thesis. As stated above, Dr. Willmore's contributions included formulating hypothesis, experimental design, data analysis and manuscript preparation for all four manuscripts included in this thesis. Leonid Chepelev calculated bond dissociation enthalpy value for BPA and Charlie Chen, Kylie Scoggan and Melanie Coughlan (scientists from Health Canada) performed the real-time qPCR experiments on the material provided from our laboratory; these workers contributed to the research described in Chapter 4. The USC LA group described above was involved in planning experiments, presented in Chapter 5 of this thesis, including animal care and treatment while my role in this was to work with the lung tissue from these animals to perform the final set of the experiments. The contribution of other co-authors, undergraduate students from the Willmore lab, is acknowledged above. 


\section{The Acknowledgement of the Use of Copyright Material}

As an author with the Elsevier publisher, I retain the right to "include the journal article, in full or in part, in a thesis or dissertation". This statement can be found at the publisher's website (http://www.elsevier.com/wps/find/authorshome.authors/copyright) In addition, I have obtained the permission from each of my collaborators to present our collaborative work in the thesis. 


\section{Acknowledgements}

First of all, I would like to thank Carleton University, its founders, community of students and professors, for creating a unique, stimulating environment, which I have enjoyed for almost ten years since 2002. Next, I am very grateful to my supervisor, Dr. Bill Willmore, for giving me a lot of freedom during the completion of this thesis, for his guidance, support and willingness to help me learn new techniques and develop as a researcher. In addition, I thank Dr. Willmore for his literally parental support in many situations, including giving me frequent rides home, which saved me a lot of time that would be otherwise spent in tiresome trips back home, for bringing me to several top scientific conferences and coming up with the funds when the financial times were tough for me. The guidance of my advisors, Dr. Susan Aitken and Dr. Michael Jonz is very much appreciated; they helped maintain confidence in what I was doing. I am grateful to the Society for Free Radical Biology and Medicine (SFRBM) for their long interest in my work, which was recognized by the SFRBM travel award (2006), SFRBM Young Investigator Award (2009) and the SFRBM Mini-Fellowship (2010), which allowed me to learn new techniques at the University of Southern California, Los Angeles, under the supervisory guidance of Dr. Henry Forman, whose enthusiasm and sense of humor was of great support and encouragement. An ideal working environment was provided by Willmore lab with many hard-working undergraduate students such as Joshua Bennitz, Julia DiLabio, Ting Huang, Skye and Arran McBride and Andrew Seal. Last, but not least, a great deal of help and encouragement came from my mother Lioudmila and my brother Leonid, who believed in me and fostered my personal growth and development. 


\section{Table of Contents}

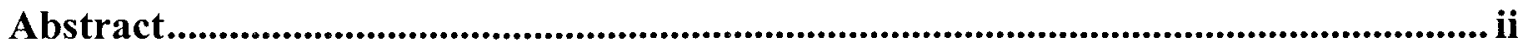

Preface

Acknowledgements ........................................................................................................................... viii

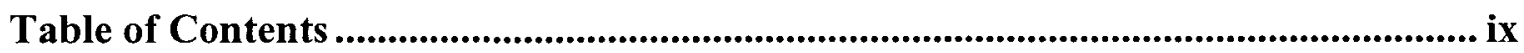

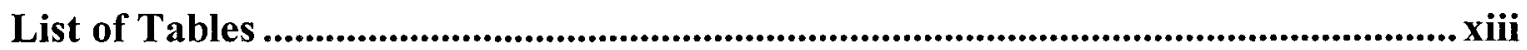

List of Figures.............................................................................................................................. xiv

List of Appendices..................................................................................................................... xvi

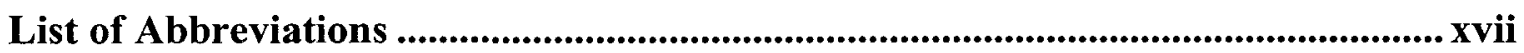

Chapter 1: General Introduction ................................................................................................ 21

1.1 Hypoxia, its Challenges and Main Regulatory Proteins..................................... 22

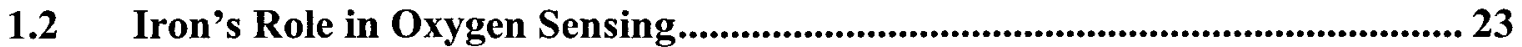

1.3 The Generation of Reactive Oxygen Species is Associated With Both Iron

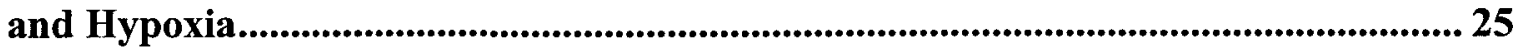

1.4 The CNC-bZIP Transcription Factor Subfamily Includes Nrf1 and Nrf2 ... 27

1.5 Heme Oxygenase-1 is a Hypoxia- and Oxidative Stress-inducible Enzyme .. 32

1.6 Nrf1 Regulation Differs From That of Nrf2 ........................................................ 35

1.7 ROS, Nrf2 and Their Potential Involvement in Aging...................................... 48

1.8 Bisphenol A, the Mechanism of Its Action in Biological Systems and Its

Relation to Oxygen Metabolism and Antioxidant Enzymes .......................................... 49 
1.9 Research Hypotheses

Chapter 2: Methods

2.1 Justification for the Use of Specific Cell Lines and Experimental Design..... 55

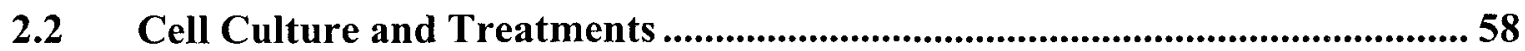

2.3 Immunoblotting and Co-immunoprecipitation...................................... 58

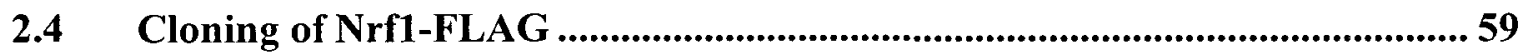

2.5 Transient Transfections and Luciferase Reporter Assays ........................... 59

2.6 Electrophoretic Mobility Gel Shift Assays .............................................. 61

2.7 Computational and Bioinformatics Analyses .........................................61

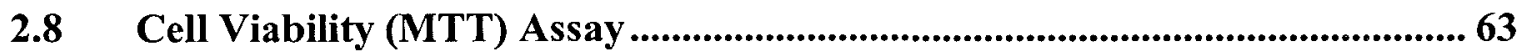

2.9 ROS, Glutathione and Protein Carbonyl Assays ....................................... 64

2.10 Total RNA Isolation and Real-time Quantitative PCR (real-time qPCR) .... 64

2.11 Nanoparticle Production, Mouse Exposure and Tissue Processing ............... 65

2.12 Oxygen Radical Absorbance Capacity (ORAC) Assay ............................. 67

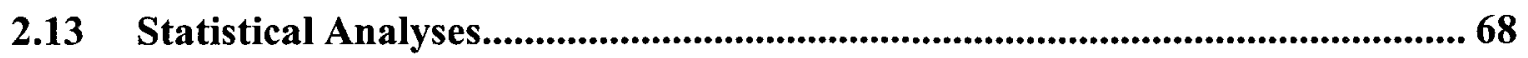

Chapter 3: Oxygen-dependent and Proteasomal Regulation of Nrf1 ........................ 69

3.1 Proteasomal Inhibition Stabilizes p120 Nrf1 and Ubiquitinated Nrf1 ......... 70

3.2 Nrf1 Contains a PEST Motif and is Degraded by the Proteasome ................ 77

3.3 Nrf1 Protein Expression under Proteasomal Inhibition and Hypoxia .......... 84

3.4 MG-132 and Hypoxia Enhances Protein Binding to the ARE...................... 89

3.5 Hypoxia Activates and Proteasomal Inhibitors Inhibit Exogenous Nrf1

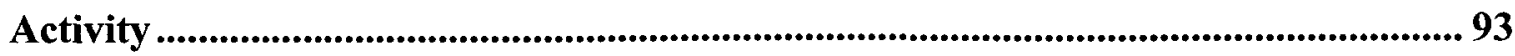

3.6 Potential Effect of p65 Nrf1 on the Hypoxic Inducibility of Nrf1 ................96 
3.7 Nrf1 is Activated by Phosphorylation

3.8 The Potential Involvement of ROS in Hypoxic Inducibility of Nrf1 .......... 100

3.9 The Two Proposed Levels of the Proteasomal Control of Nrf1 ................. 100

3.10 Potential Significance of the Stress-inducible Forms of Nrf1 .................... 104

3.11 Hypoxic Activation of Nrf1 is Accompanied by p65 Nrf1 Down-regulation 104

3.12 The Proposed Model for the Nrf1 Regulation by Hypoxia and the

Proteasome 106

Chapter 4: The Effect of BPA on the Nrf1/2-ARE Pathway 109

4.1 BPA and its Relation to the ROS Generation and Antioxidant Enzymes ... 110

4.2 BPA Promotes Cell Proliferation and Reduces Basal ROS Levels 112

4.3 BPA Alters Protein Binding to the ARE and Activates Nrf1 and Nrf2 ...... 116

4.4 BPA Upregulates the Nrf1/2-ARE Pathway-controlled Genes................... 122

4.5 BPA Downregulates the Protein Expression of Nrf1, Nrf2 and HO-1 ........ 123

4.6 The Proposed Model for the Effect of BPA on the Nrf1/2-ARE Pathway... 130

Chapter 5: Nrf1 Regulation in Aging Mice 136

5.1 Aging, Air Particulate Exposure and their Relation to the ROS Generation,

Nrf1 and Nrf2 137

5.2 Nrf1 Silencing Activates the Expression of the ARE-target Genes 140

5.3 Exogenous Nrf1 Suppresses Nrf2 Induction by tert-Butylhydroquinone (tBHQ) 144

5.4 The DNA-binding Activities of Nrf1 and Nrf2 Diminish with Age .............. 145

5.5 Nrf1 and Nrf2 Protein Levels Change in the Opposing Fashion with Age . 148 
5.6 The Inducibility of the Nrf1/2-ARE-controlled Genes and the Cellular

Antioxidant Defence Capacity is Impaired with Age 148

5.7 The Proposed Model for the Effect of Aging and Particulate Exposure on the Nrf1 and Nrf2 Function in Mice 149

Chapter 6: General Discussion and Conclusions 163

6.1 Towards Understanding the Physiological Functions of Nrf1 164

6.2 Assessment of the Current Models to Study Nrf1 Functiong 167

6.3 The Potential of Manipulating Nrf1 Activity for Clinical Purposes 171

6.4 Conclusions and Future Directions 173

Appendices. 183

Appendix A 183

A.1 List of Publications 183

A.2 Ten Simple Rules for Surviving Graduate School 186

References 189 


\section{List of Tables}

Table 4.1 Enhanced Expression of the Nrf1/2-ARE Pathway Regulatory and Target Genes at Different BPA Concentrations ........................................................... 125 


\section{List of Figures}

Figure 1.1. The CNC-bZIP Transcription Factors Regulate the AntioxidantResponse Element (ARE)/Electrophile-Response Element (EpER)-driven Gene Expression

Figure 1.2. The Canonical Model for the Nrf2 Regulation by the Proteasomal Degradation

Figure 1.3. The Comparison Between the Nrf2 and Nrf1 Domains

Figure 1.4. The Structural Domains in Human Nrf1 (hNrf1) and the Predicted Topology of Nrf1 Within the ER membrane. 45

Figure 3.1. Nrf1 is Stabilized by Proteasomal Inhibition in COS7 Cells 74

Figure 3.2. Oxidative Stressor AAPH Induces Nrf1 DNA Binding and Stabilizes p95 Nrf1 Form Independently of p120.

Figure 3.3. Nrf1 is a Short-lived Protein, Undergoes Ubiquitination and Proteasomal Degradation 80

Figure 3.4. Oxidative Stressors AAPH and tBHQ, Hypoxia, CHX and Aging Destabilize the p250 Form of Nrf1 83

Figure 3.5. Comparison of the Nrf1 Expression under Hypoxia and Proteasomal Inhibition

Figure 3.6. Hypoxia and Proteasomal Inhibition Increase Protein Binding to the ARE

Figure 3.7. Hypoxia and Hypoxic Mimetics Activate Nrf1, While MG-132 Inhibits

Its Transactivation Activity 95

Figure 3.8. Phosphorylation Activates Nrf1 and Antioxidant Treatment only Partially Abolishes the Hypoxic Inducibility of Nrf1 98

Figure 3.9. The Proposed Working Model for the Nrf1 Regulation by the Proteasome and Oxygen 109

Figure 4.1. Theoretical Pro-oxidant and Antioxidant Properties of BPA 113

Figure 4.2. The Effect of BPA on Cell Viability and Intracellular Redox Status ... 116 
Figure 4.3. BPA Alters Protein Binding to ARE Consensus Sequence Probe......... 120

Figure 4.4. BPA Activates Exogenous Nrf1 and Nrf2 in HEK 293 Cells

Figure 4.5. Protein Expression of the Nrf1/2-ARE Pathway Regulatory (Nrf1 and

Nrf2) and the Target Proteins (HO-1 and Nqo1) as a Result of BPA Treatment .. 128

Figure 4.6. HO-1 Putative Dimer Formation as a Result of BPA Treatment 129

Figure 4.7. The Proposed Model for the BPA Effect on the ARE-Nrf1/2 Pathway 135

Figure 5.1. Nrf1 Silencing Activates the Expression of the ARE-target Genes...... 143

Figure 5.2. The Full-length (p120Nrf1) and 65-kDa (p65Nrf1) Forms of Nrf1 Suppress the Nrf2-mediated Transcription of ARE-controlled Gene Expression.. 147

Figure 5.3. Aging is Accompanied by Decreased Protein Binding to the ARE...... 157

Figure 5.4. Nrf1 and Nrf2 Protein Levels Change in the Opposing Fashion With Age 159

Figure 5.5. Age-dependent Decline in the Inducibility of the Nrf1/2-controlled Gene Expression and Oxygen Radical Scavenging Capacity

Figure 5.6. The Proposed Model for the Combined Effect of the Particulate Exposure and Aging on the Nrf1/2-ARE Pathway 162

Figure 6.1. The Progress of Our Understanding of the Nrf Factors as Revealed by the Number of PubMed Publications. 164

Figure 6.2. The Experimental Design Determines the Confidence in the Results of Transcription Factor Studies

Figure 6.3. The Amino Acid Sequence of Nrf1 Along with Its Domains and Features 


\section{List of Appendices}

Appendix A..................................................................... 183

A.1 List of Publications................................................... 183

A.2 Ten Simple Rules for Surviving Graduate School ........................ 186 


\section{List of Abbreviations}

AA - Amino Acid

AAPH - 2,2'-azobis(2-amidinopropane) Dihydrochloride

AD - Acidic Domain

ALAD - $\delta$-Aminolevulinate dehydratase

ALAS2 - Erythroid-specific 5-Aminolevulinate Synthase

ARE - Antioxidant Response Element

Bach - BTB (Broad-complex, Tramtrack, and Bric-a-brac) and CNC (Cap'n'Collar)

Homology 1, Basic Leucine Zipper Transcription Factor

BDE - Bond Dissociation Energy

BPA - Bisphenol A

BSO - Butathione Sulfoxamine

bZIP - Basic Leucine Zipper

Cp - Ceruloplasmin

CHX - Cycloheximide

CNC-bZIP - Cap'n'Collar-Basic Leucine Zipper

Co-IP - Co-immunoprecipitation

COS - African Green Monkey-derived, SV40-transfected Cells

CPRG - Chlorophenol Red- $\beta$-D-galactopyranoside

DMEM - Dubecco's Modified Eagle's Medium

DNA - Deoxyribonucleic Acid

DMOG - Dimethyloxalylglycine

DMSO - Dimethylsulfoxide 
DTT - Dithiothreitol

EDTA - Ethylenediaminetetraacetic Acid

EMSA - Electrophoretic Mobility Shift Assay

EPR - Electron Paramagnetic Resonance

EpRE - Electrophile Response Element

ER - Endoplasmic Reticulum

ERAD - ER-associated Protein Degradation

ETC - Electron Transport Chain

FLAG - An Octapeptide Tag C-DYKDDDDK-N

FCS - Fetal Calf Serum

FECH - Ferrochelatase

FTL - Ferritin Light Chain

FTH - Ferritin Heavy Chain

GAPDH - Glyceraldehyde-3-phosphate Dehydrogenase

Gclm - Glutamate-cysteine Ligase Modifier Subunit

Gclc - Glutamate-cysteine Ligase Catalytic Subunit

GS - Gas Chromatograph

GSH - Reduced Glutathione

GSSG - Oxidized Glutathione

H2DCFDA - 5-(and-6)-Carboxy-2',7'-dichlorodihydrofluorescein Diacetate

HEK - Human Embryonic Kidney Cells

HEPES - 4-(2-hydroxyethyl)-1-piperazineethanesulfonic Acid

HIF - Hypoxia-Inducible Factor 
hFLF - Human Fetal Lung Fibroblast Cells

HMBS - Porphobilinogen Deaminase

HO-1 - Heme Oxygenase-1

HS - Horse Serum

HRP - Horseradish Peroxidase

IgG - Immunoglobulin G

IRP - Iron-response Proteins

kDa - KiloDalton

Keap1 - Kelch-like ECH (Erythroid Cell-derived Protein with Cap'n'Collar Homology)-Associated Protein1

LIP - Labile Iron Pool

LXXLAP - Oxygen-dependent Degradation Motif

MAPK - Mitogen-activated Protein Kinase

Mf - Mitoferrin

MT - Metallothionein

MTT - Methylthiazolyldiphenyl-tetrazolium Bromide

MSD - Mass Selective Detector

MW - Molecular Weight

NAC - N-acetylcysteine

NCS - Newborn Calf Serum

Neh6L - Neh (Nrf2-ECH homology) 6-like Domain

NO - Nitric Oxide

Nrf - Nuclear Factor-Erythroid 2 p45 Subunit-related Factor 
NST - Asn/Ser/Thr-rich Domain

NTD - N-terminal Domain

OA - Okadaic Acid

p45NFE2 - Nuclear Factor-erythroid 2 p45 Subunit

p250, p140, p120, p95, p65, p23 - Various Forms of Nrf1 Protein

PBS - Phosphate Buffered Saline

PEST - an Amino Acid Region Rich in Pro, Glu, Thr and Ser

PHD - Prolyl Hydroxylase

PKC - Protein Kinase C

PMSF - Phenylmethanesulfonylfluoride

PM - Particulate Matter (Nanoparticles with a Diameter $<200 \mathrm{~nm}$ )

qPCR - Quantitative PCR

Rac - Rho Family GTPase

RNA - Ribonucleic Acid

RNAi - RNA Interference

ROOH - Peroxyl

ROO• - Peroxyl Radical

ROS - Reactive Oxygen Species

SR - Ser Repeat Domain

Src - Sarcoma Protein Kinase

tBHQ - tert-Butylhydroquinone

Tf - Transferrin

TfR - Transferrin Receptor 
1 Chapter: General Introduction 


\subsection{Hypoxia, its Challenges and Main Regulatory Proteins}

Hypoxia constitutes a stress for all mammalian cells. It is encountered at high altitudes, during prenatal development, as well as in some pathophysiological conditions such as ischemia, cardiovascular and pulmonary diseases, cancer and sleep apnea. Mammalian cells have evolved complex mechanisms to "sense" changes in ambient oxygen concentrations and mount appropriate adaptive responses to return to a state of homeostasis. It should be emphasized that different cells and tissues in situ are exposed to variable oxygen levels (about 2-5 \% $\mathrm{O}_{2}$ (Stiehl et al., 2006) compared to $21 \%$ air oxygen concentration typically used in cell culturing). Therefore, the term "hypoxia" is relative and attempts to describe oxygen concentrations significantly lower than normal, physiological levels to which a given cell type, tissue or organ is exposed.

Mammals cope with hypoxia by: 1) increasing the oxygen-carrying capacity and rate of blood flow to hypoxic organs and tissues; and 2) switching from aerobic (i.e. oxidative phosphorylation) to anaerobic (e.g. glycolysis) metabolic pathways. The former involves activating gene and protein expression responsible for erythropoiesis (erythropoietin), angiogenesis (vascular endothelial growth factor), vasodilation (NO synthase), iron transport (transferrin (Tf), Tf receptor (TfR), ceruloplasmin $(\mathrm{Cp})$ and mitoferrin (Mf)), storage (ferritin) and heme turnover (erythroid-specific 5aminolevulinate synthase (ALAS2), ferrochelatase (FECH) and heme oxygenase-1 (HO1)). The latter strategy relies on the up-regulation of key glycolytic enzymes, including 
aldolase A, glucose-6-phosphate isomerase, phosphoglycerate kinase 1 and pyruvate kinase M (Semenza et al., 2004; Naughton, 2003).

These adaptations are primarily mediated by the key regulatory proteins of iron metabolism, hypoxia-inducible factors (HIFs) and iron-response proteins 1 and 2 (IRP1 and IRP2). These regulatory proteins, in turn, appear to be the targets of oxygendependent modifications of their structure and function, which allows HIFs and IRPs to act as "molecular switches" for oxygen sensing, response and adaptation, regulating transcription (HIFs) or translation (IRPs) of their targets. In addition, Cap'N'Collar basic Leu Zipper (CNC-bZIP) transcriptional factors appear to be important gene expression activators and repressors of $\mathrm{HO}-1$, ferritin and $\beta$-globin as well as cellular antioxidant defense and detoxification system genes.

\subsection{Iron's Role in Oxygen Sensing}

Iron $(\mathrm{Fe})$ is intimately associated with oxygen sensing in cells. Since Fe is indispensable to oxygen transport, its shortage can create low oxygen conditions (hypoxia) as a result of diminished $\mathrm{O}_{2}$ delivery to cells and tissues. Both $\mathrm{Fe}$ and $\mathrm{O}_{2}$ depletion may compromise ATP production by the electron transport chain (ETC) and thus both deficiencies may trigger similar signaling pathways. Given close association between $\mathrm{O}_{2}$ and $\mathrm{Fe}$ homeostasis, it was proposed that the heme moiety of some heme proteins could function as an oxygen sensor (Goldberg et al., 1988). According to this early hypothesis, a liganddependent change in the conformation of this heme moiety was responsible for oxygen 
sensing based on the fact that hypoxia-inducible induction of the erythropoietin gene was abolished by $\mathrm{CO}$, which binds to heme iron strongly and selectively, as well as by heme synthesis inhibitors. Soon after the discovery of hypoxia-inducible factor-1 (HIF-1), a master regulator of hypoxic gene expression (Wang and Semenza, 1993), this hypothesis was modified to explain the role of the enigmatic heme protein in the context of HIF-1 stabilization and activation during hypoxia. It was envisioned that reversible $\mathrm{O}_{2}$ binding to the $\mathrm{Fe}$ atom of the heme protein resulted in the production of superoxide, $\mathrm{O}_{2}^{-}$, subsequently convertible to other reactive oxygen species (ROS) using $\mathrm{Fe}^{2+}$ as a catalyst and that ROS were capable of oxidizing HIF-1, rendering it inactive under normoxia (Bunn and Poyton, 1996). During hypoxia, however, the levels of ROS were thought to be diminished, resulting in stabilization of HIF-1 and its activation of hypoxia-inducible gene expression. After two landmark publications on the role of prolyl hydroxylases (PHDs; iron-binding proteins) in controlling HIF levels (Ivan et al., 2001; Jaakkola et al., 2001), the idea that an oxygen sensor molecule is a heme protein became less noticeable in the literature. Despite this, Fe still occupies a central role in $\mathrm{O}_{2}$ sensing due to its direct involvement in PHD-catalyzed HIF hydroxylation. It was further suggested that hypoxic elevation of ROS (generated probably by the increased half-life of reactive semi-quinone intermediates at complex III of the electron transport chain; see Klimova and Chandel, 2008) could affect the oxidation status of iron bound to PHD, interfering with PHD function during hypoxia (Kaelin, 2005), perhaps through the Src- (Sarcoma) protein kinase Rac (Rho family GTPase) dependent activation of mitogen-activated protein kinase (MAPK, Bell et al., 2005). Unlike the earlier hypothesis, which asserted that diminished ROS production during hypoxia activated HIF (Wang and Semenza, 1993), 
the current view on the potential involvement of increased ROS in PHD inactivation and hypoxic HIF stabilization (Kaelin, 2005) relies on more recent evidence of increased ROS generation by complex III of the mitochondrial ETC during hypoxia (Guzy et al., 2005; Brunelle et al., 2005; Bell et al., 2007). To date, Rieske Fe-S (iron-sulphur) proteins, PHDs, and heme-recycling enzyme heme oxygenase 2 (HO-2) have all been described as putative oxygen sensing systems ((Guzy et al., 2005; Brunelle et al., 2005; Bell et al., 2007; Acker et al., 2006; Fähling et al., 2009). Remarkably, all of these proposed $\mathrm{O}_{2}$ sensors are iron-binding or heme-binding proteins.

\subsection{The Generation of Reactive Oxygen Species is Associated With Both Iron} and Hypoxia

At physiological $\mathrm{pH}$, ferrous $\left(\mathrm{Fe}^{2+}\right)$ ion spontaneously oxidizes to produce ROS including superoxide, hydrogen peroxide and the highly reactive hydroxyl radical, capable of covalently damaging proteins, lipids and DNA (Halliwell and Gutteridge, 2007). The prooxidant capacity of iron has long been recognized and the most famous example is socalled "Fenton reaction" in which iron reacts with hydrogen peroxide to generate the hydroxyl radical according to the following equation:

$$
\mathrm{Fe}^{2+}+\mathrm{H}_{2} \mathrm{O}_{2} \rightarrow \mathrm{Fe}^{3+}+\mathrm{HO}^{\bullet}+\mathrm{HO}^{-}
$$

The propensity of iron to generate ROS, inducing oxidative damage, is thought to contribute to the development and progression of some neurodegenerative diseases such 
as Alzheimer's, Parkinson's and Huntington's disease (Altamura and Muckenthaler, 2009). Iron for Fenton reactions comes from the intracellular redox-active iron pool, also known as the labile iron pool (LIP). In most organisms, LIP is kept small and does not constitute a major source of ROS (Breuer et al., 1997). Iron within LIP is thought to exist mostly in ferric form, complexed with citrate or ADP, with a small fraction of LIP consisting of cytotoxic ferrous iron (Kohgo et al., 2008). The level of LIP reflects the overall iron status of the cell (Galaris and Pantopoulos, 2008). Due to its toxicity, LIP is maintained only as a minor fraction (3-5\%) of the total cellular iron (Kruszewski, 2003). Thus it was found that catalytic iron, available for Fenton reactions, is in the micromolar range in the plasma of diseased patients and is undetectable in healthy people (see (Halliwell, 2009) and references therein). To diminish pro-oxidant activity of iron, many organisms employ iron sequestration since protein-bound iron displays a decreased tendency to generate ROS and induce oxidative damage (Halliwell and Gutteridge, 2007). However, if the iron concentrations exceed the capacity of iron-detoxification systems (iron transport, sequestration and oxidation of toxic $\mathrm{Fe}^{2+}$ to less reactive $\mathrm{Fe}^{3+}$ ), excessive production of hydroxyl and lipid radicals takes place as a direct consequence of $\mathrm{Fe}^{2+}$ excess (Rothman et al., 1992). Ironically, in addition to iron excess, iron deficiency can also contribute to oxidative damage as a result of increased electron leakage from the faulty iron-dependent components of mitochondrial ETC (Halliwell and Gutteridge, 2007).

Similar to iron deficiency or overload, hypoxia has also been known to contribute to increased rates of ROS formation (see Klimova and Chandel, 2008) for a 
comprehensive review). Approximately 1 to $5 \%$ of the oxygen used for oxidative phosphorylation is converted to superoxide as a result of incomplete reduction of oxygen to water by the ETC. There is a large body of evidence suggesting that hypoxia increases superoxide production from complex III of ETC (possibly by increasing the life-time of hydroquinone intermediate (Andreini et al., 2009; Chandel et al., 2000; Guzy et al., 2007). Superoxide is then converted to hydrogen peroxide in the cytosol, activating oxygen-dependent signaling pathways responsible for HIF $\alpha$ stabilization (Klimova and Chandel, 2008; Aragonés et al., 2008). HIF, in turn, activates the hypoxic gene expression of major proteins involved in iron metabolism.

\subsection{The CNC-bZIP Subfamily of Transcriptional Factors Include Nrf1 and Nrf2}

The CNC-bZIP factors include the Nuclear Factor-Erythroid 2 p45 Subunit-related Factors (Nrf1, Nrf2, Nrf3, NF-E2 p45), and more distant members Bach1 and Bach2. These play an important role in regulating the expression of such iron-related genes as ferritin (both light (FTL) and heavy (FTH) chains), $\beta$-globin and HO-1. By binding to an enhancer element known as the Antioxidant Response Element (ARE; consensus sequence: TGCTCAGTCAT; also referred to as the Electrophile Response Element, EpRE), Nrfl, Nrf2, Nrf3 and NF-E2 p45 activate ARE-driven gene expression under various physiological conditions (Figure 1.1). Bach1 and Bach2 act as dominant repressors of ARE-driven gene expression by heterodimerizing with the Maf proteins on the ARE (Dhakshinamoorthy et al., 2005; Sun et al., 2002; Igarashi et al., 1998). 
The ARE sequence is very similar to the Maf recognition element (MARE) sequence such that the terms "ARE" and "MARE" are often used interchangeably (Igarashi and Sun, 2006). The CNC-bZIP transcriptional factors bind to AREs as heterodimers with small Maf proteins (for review see (Motohashi et al., 2002). Interestingly, small Maf-NF-E2 p45, Maf-Nrf1, Maf-Nrf2 or Maf-Nrf3 heterodimers activate while small Maf-Bach1 heterodimers repress the expression of $\beta$-globin and ho-1 genes (Sun et al., 2004). Thus, cells possess the ability to fine-tune the expression of iron-related genes, either by activation or repression, through the same ARE regions bound by different members of the same subfamily of proteins.

It has been extensively demonstrated that hypoxia leads to increased mitochondrial ROS production (see Klimova and Chandel (2008) for a review), although some controversy regarding whether ROS concentrations increase during hypoxia still exists. Therefore, it seems reasonable to hypothesize that hypoxic response and antioxidant response pathways are connected and some of our observations point out to the existence of an interesting link between hypoxia and the antioxidant response pathway. It will be discussed in greater detail further that using a luciferase reporter vector with three ARE elements from chicken $\beta$-globin enhancer $(3 \times$ ARE-luciferase (Igarashi et al., 2004)), we noticed that the luciferase activity upon transient cotransfection of Nrfl-FLAG and $3 \times$ ARE-luciferase was significantly higher during hypoxia treatment of COS7 and HEK293 cells (Chepelev et al., submitted). Interestingly, hypoxic induction of $\mathrm{Nrfl}$ was abolished by antioxidant $\mathrm{N}$-acetylcysteine. Using a similar approach, we were able to show hypoxic inducibility of Nrf2 as well. This suggests that 
hypoxia activates $\mathrm{Nrf1}$ and $\mathrm{Nrf} 2$ transactivation activity through ROS signaling pathways, potentially inducing gene expression of ferritin, ho-l and other AREcontrolled genes. 


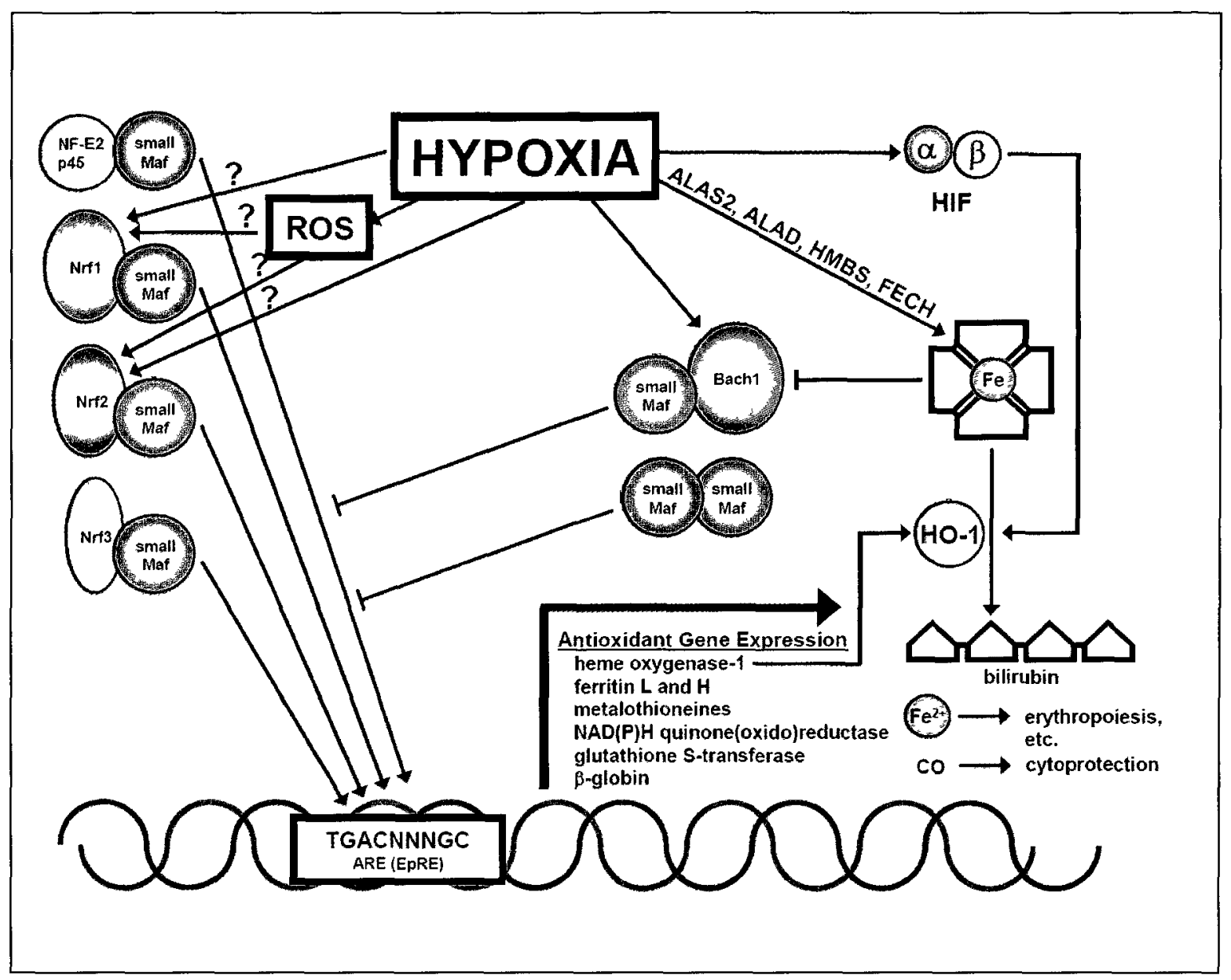


Figure 1.1. The CNC-bZIP Transcription Factors Regulate Antioxidant-Response Element (ARE)/Electrophile-Response Element (EpER)-driven Gene Expression. The CNC-bZIP trancription factors are located at the integration point of the oxygen and iron metabolism regulatory pathways. CNC-bZIP factors include activators Nrf1, Nrf2, Nrf3, NE-F2 p45 and repressors Bach1 and Bach2 and bind to ARE (EpRE) as heterodimers with small Maf proteins. Hypoxia induces heme synthesis and heme dislodges Bach1 from its interaction with Maf-occupied AREs, favoring the binding of activators (Nrfl, 2 and 3) to the ARE and activating the expression of ferritin, HO-1 and $\beta$-globin genes. Hypoxia is known to stabilize the $\alpha$ subunit of heterodimeric HIF factors and upregulate the expression of genes and/or proteins involved in iron transport and metabolism, many of which also contain functional AREs (e.g., ferritin, heavy and light chains, $\beta$-globin and HO-1). Cell type- and tissue-specific upregulation of heme synthesis in erythroid-related cells and augmented heme degradation in other cell types can help the process of "iron redistribution" during hypoxic challenge such that more red blood cells can be produced to increase the oxygen-carrying capacity of the blood. We hypothesize that hypoxia activates $\mathrm{Nrf1}$ and Nrf2 transactivation through ROS pathways. ALAS2, erythroid-specific aminolevulinate synthase; ALAD, $\delta$-aminolevulinate dehydratase; HMBS, porphobilinogen deaminase; FECH, ferrochelatase. Taken from Chepelev and Willmore (2011). 
Antioxidant and cytoprotective genes, regulated transcriptionally through their AREs, include $\mathrm{NAD}(\mathrm{P}) \mathrm{H}$ :quinone oxidoreductase 1 (nqol), the glutathione-Stransferases (gst), ferritin light $(f t)$ and heavy $(f t h)$ chains, heme oxygenase-1 (ho-l), catalase (cat) and superoxide dismutase (sod) (Jung and Kwak, 2010). Since Nrfl controls phase 2 detoxification enzymes that aid in metabolism and removal of potential carcinogens, and due to the fact that potent ARE-inducers such as sulforaphane are known chemopreventive agents, understanding the mechanisms of Nrfl regulation may aid in the development of cancer therapeutics. Furthermore, due to the cytoprotective nature of phase 2 enzymes against oxidative stress-induced neurodegeneration (Satoh et al., 2009), manipulation of the upstream factors controlling these enzymes (e.g., Nrfl and $\mathrm{Nrf2)}$ could be useful in the search of therapeutic targets against chronic neurodegenerative diseases (Satoh et al., 2009).

\subsection{Heme Oxygenase-1 is a Hypoxia- and Oxidative Stress-inducible Enzyme}

Heme oxygenase-1 (HO-1; E.C. 1.14.99.3) is the major enzyme involved in the recycling of heme and is controlled by multiple mechanisms. It catalyzes the first and rate-limiting step of heme breakdown by cleaving the heme ring at the alpha methane bridge to form biliverdin (heme $+\mathrm{NAD}(\mathrm{P}) \mathrm{H}+\mathrm{H}^{+}+3 \mathrm{O}_{2} \leftrightarrow$ biliverdin $+\mathrm{Fe}^{2+}+\mathrm{CO}+\mathrm{NAD}(\mathrm{P})^{+}+3 \mathrm{H}_{2} \mathrm{O}$ ). The central iron of heme substrate is kept in reduced state by $\mathrm{NAD}(\mathrm{P}) \mathrm{H}$. Since the majority of the iron utilized for hypoxia-inducible heme synthesis comes from cellular hemoproteins (Peyssonnaux et al., 2008), heme needs to be recycled at a higher rate in low oxygen conditions to satisfy the growing need for iron, required for heme synthesis 
in the bone marrow, in order to stimulate the production of red blood cells; therefore, heme degradation enzymes are expected to be up-regulated in response to erythropoietin stimulation to keep up with the increased iron demand (Soe-Lin et al., 2008).

The human HO-1 protein is composed of 288 amino acids and its expression is increased by its heme substrate (Yoshida et al., 1988) as well as by heavy metals, bromobenzene, endotoxins, cytokines, hypoxia, oxidative stress and UV radiation (Maines, 1992). It is located in the endoplasmic reticulum (ER) and is generally thought to exist as a homodimer in most organisms where it has been characterized; however, some recent work (Hwang et al., 2009) suggests that HO-1 monomers form homodimers and even oligomers in the ER.

Hypoxia has long been known to affect HO-1 expression (for a review, see Shibahara et al., 2007). The general response of HO-1, however, appears to be cell- and species-specific. Hypoxia repressed ho-l mRNA expression in primary cultures of human umbilical vein endothelial cells, human astrocytes, and human coronary artery endothelial cells (Nakayama et al., 2000). On the other hand, hypoxia increased HO-1 expression in human lung A549 cells, which was markedly attenuated by HIF-1 $\alpha$ knockdown (Hänze et al., 2003). Hypoxia also induced HO-1 in rat liver (Bonkovsky et al., 1986), heart (Katayose et al., 1993), as well as in cultured animal cells, including Chinese hamster ovary cells (Murphy et al., 1991), rat ventricular smooth muscle cells (Lee et al., 1997; Morita et al., 1995), and rat myocytes (Eyssen-Hernandez et al., 1996). In mice, a 163 kilobase pair (kb.p.) hypoxia-responsive region is found approximately $9.5 \mathrm{~kb} . \mathrm{p}$. away 
from the transcriptional start site of the ho-l gene and contains two functional HREs (Lee et al., 1997). Induction of HO-1 by HIF during hypoxic conditions was found to be cardioprotective (Czibik et al., 2009) and provided protective effects in retinal ischemia (Zhu et al., 2007). In the human ho-1 gene, three putative HREs are found from -121 to 126 (CACGTG) and $-5,619$ to $-5,624$ upstream (TACGTG) as well as $+1,812$ to $+1,817$ downstream (AACGTG) from the transcriptional start site (Willmore et al., unpublished results). Whether these are functional HREs remains to be tested.

A potential hypoxia-inducible repression of ho-l gene expression by Bach1, a heme- and an ARE-binding protein, has been reported (Kitamuro et al., 2003). An ARE is found in the human ho-1 gene, which is not surprising given that HO-1 is regarded as an antioxidant enzyme (Levonen et al., 2007) which removes potentially cytotoxic heme, produces an antioxidant billirubin and carbon monoxide that confers proper regulation of vascular functions. The ho-l ARE is required for Bachl-mediated repression of a reporter gene by hypoxia in human glioblastoma, lung cancer and umbilical vein endothelial cells (Kitamuro et al., 2003). It appears that CNC-bZIP-ARE and HIF-HRE controls could have some overlapping functions in terms of HO-1 regulation as reported by Lee et al. (1997). These workers observed marked HIF-dependent increase of ho-1 mRNA in whole rats and cultured rat cells treated with hypoxia. Interestingly, treatment with cobalt, which mimics the hypoxic condition, led to the accumulation of ho-l mRNA in both wildtype and HIF-deficient Chinese hamster ovary cells (Gong et al., 2001), suggesting that HO-1 expression is controlled at least at two different levels. Again, multiple ways of HO-1 control may allow cells to fine-tune gene expression during various oxygen and 
iron conditions. In addition, increased hypoxic expression of HO-1 in vascular systems produces $\mathrm{CO}$, thought to confer proper regulation of vascular functions and provide negative feedback on hypoxia induction (Huang et al., 1999; Ryter and Choi, 2009). I believe the work presented here describes some very interesting results regarding the HO1 dimerization, which might be helpful in further elucidating the function of this important cytoprotective enzyme, which has been a subject of numerous studies and the manipulation of which may be beneficial in molecular therapy of diseases, involving tissue injury (Ryter and Choi, 2009).

\subsection{Nrf1 Regulation Differs From That of Nrf2}

Nrf2 is a well-established master regulator of oxidative stress-inducible gene expression (Pi et al., 2010). Currently, the mechanisms controlling Nrf2 activity have been studied in great detail with over 2,000 articles catalogued in PubMed, while studies examining Nrf1 regulation are lacking, totaling only 47 PubMed articles to date. Some of the potential reasons why Nrf1 has received less attention compared to Nrf2 may include: i) the fact that, unlike $N r f 2$ global knock-out mice, $N r f l$-deficient animals are non-viable and die at the embryonic stage (Chan et al., 1998); ii) the concept that Nrf2 is responsible for the inducible ARE-driven gene expression (Ohtsuji et al., 2008), such that it can be activated and studied using multiple stimuli while Nrfl appears to be involved in the constitutive expression of the ARE target genes (Ohtsuji et al., 2008); and iii) the hypothesis that Nrfl may play a role in regulating membrane-dependent biological processes (Zhang and Hayes, 2010), expanding the function of Nrfl beyond that of a transcription factor and, 
perhaps, providing researchers with the data that can not be conceptualized easily. Despite this apparent lack of the reports on Nrfl, it has been shown that Nrfl may play just as an important role in human pathologies as Nrf2 does (Sykiotis and Bohmann, 2010). Nrf 1 is ubiquitously expressed and is found in all tissue and cell types. Given that Nrf2 regulation has been described in greater detail compared to Nrfl, the regulation of $\mathrm{Nrf2}$ can be a starting point in understanding the molecular mechanisms of the Nrf1 control. According to the established model, Nrf2 is kept in the cytosol under homeostatic conditions by interaction with Kelch-like ECH-associated protein 1 (Keap1) (Itoh et al., 1999), which acts as an E3 ubiquitin ligase adaptor and facilitates the ubiquitination of Nrf2 and its rapid degradation by the proteasome (Sekhar et al., 2002). When the cell experiences oxidative stress, the Keap1-mediated proteasomal degradation of $\mathrm{Nrf2}$ is compromised (Kobayashi et al., 2006), allowing Nrf2 to dissociate from Keap1 and translocate to the nucleus to activate ARE-driven gene expression (see Figure 1.2).

In contrast to the canonical model of $\mathrm{Nrf} 2$ regulation, more recent analysis has shown that homeostatic Keap1-Nrf2 interactions are not permanent and take place in the nucleus via transient shuttling of Keap1 into that compartment and that Nrf2 is bound to certain ARE-containing genes even under normal homeostatic conditions (Nguyen et al., 2009).

Nrf2 contains six Nrf2-erythroid-derived $\mathrm{CNC}$ homology protein (ECH) homology (Neh) domains that are highly conserved across species (Figure 1.3). The Neh1 domain is located within the CNC-bZIP domain and enables Nrf2 interaction with its 
dimerization partners, small Maf proteins. The Neh2 is an inhibitory domain of Nrf2 as it allows Nrf2 interaction with Keap1, which targets Nrf2 for proteasomal degradation. The Neh3, Neh4 and Neh5 domains are transactivation domains that bind proteins involved in the transcription, including cAMP Response Element Binding Protein (CBP) (Katoh et al, 2001). Interestingly, CBP contains histone acetyltransferase activity (Motohashi and Yamamoto, 2004), which is expected to be helpful for transcription by remodeling chromatin structure, and opens up DNA for the binding of the basal transcription machinery. Secondly, CBP is thought to act as a bridging protein that recruits the basal transcription machinery to specific sites of transcription initiation, aiding the binding of the RNA polymerase II holoenzyme to the promoter regions (Kim et al., 1998).

It is noteworthy that while $\mathrm{Nrf2}$ is regulated by rapid proteasomal degradation, mediated by Keap1, Nrf1 appears to play a role in regulating the proteasome itself. Recent studies have established Nrfl as a pivotal transcriptional regulator of the genes of subunits of the proteasome. Thus, Nrfl activates proteasome gene expression upon proteasome inhibition treatment in human Ea.hy926 cells (Steffen et al., 2010) and mouse embryonic fibroblasts (Radhakrishnan et al., 2010) to compensate for the loss of proteasome activity. Similarly, the tissue-specific Nrfl conditional knock-out in mouse brain leads to proteasomal impairment in $\mathrm{Nrfl-}$, but not $\mathrm{Nrf2}$-deficient cells, further confirming the importance of $\mathrm{Nrfl}$ as a translational regulator of proteasomal gene expression (Lee et al., 2011). 


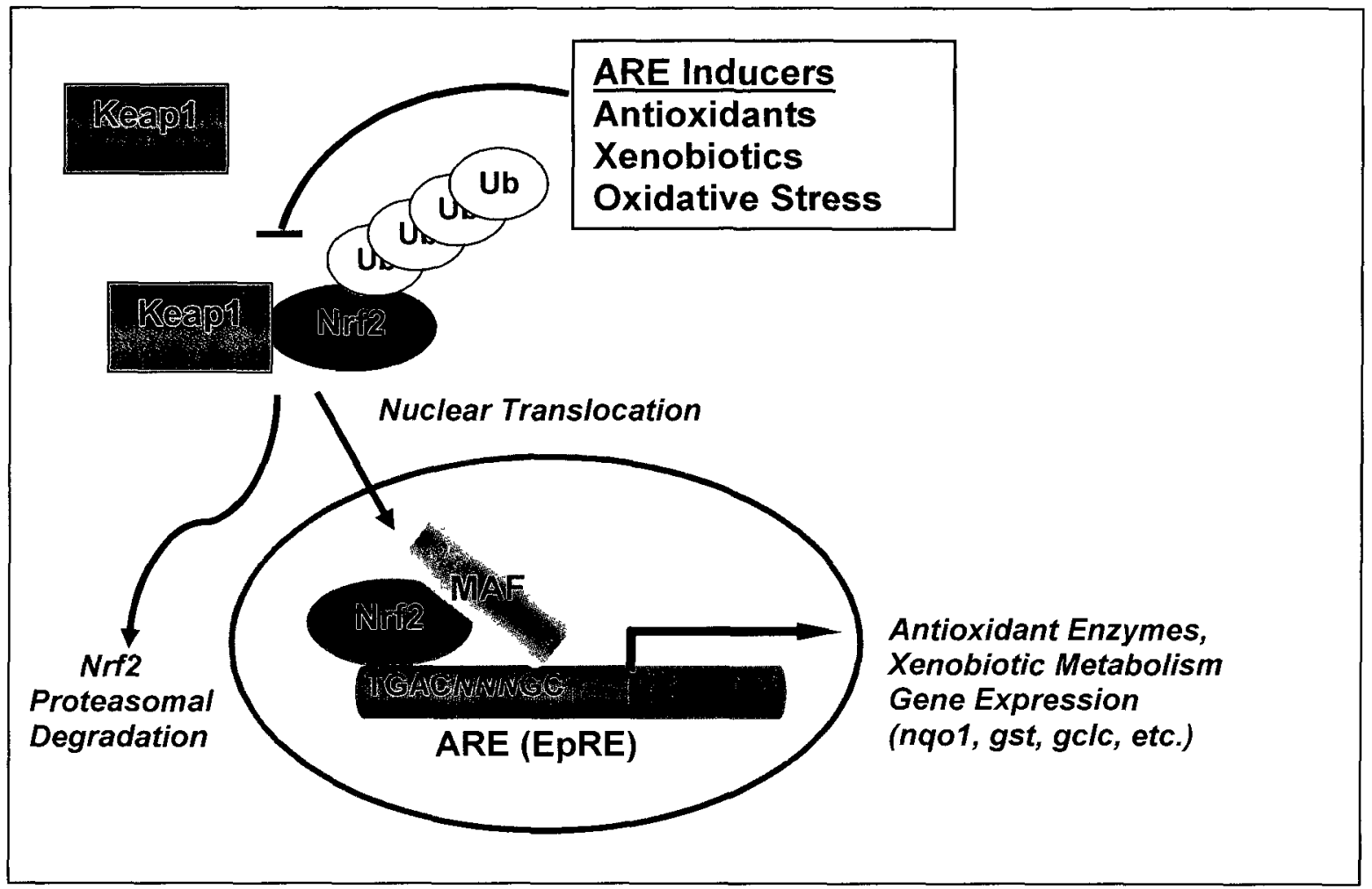

Figure 1.2. The Canonical Model for the Nrf2 Regulation by the Proteasomal Degradation. Under normal homeostatic conditions, $\mathrm{Nrf} 2$ is thought to be held in inactive state in the cytoplasm through its interaction with Keap1, which targets Nrf2 for ubiquitination and proteasomal degradation. Under appropriate stimuli, elicited by the ARE inducers and following oxidation of redox-sensitive Cys residues of Keap1, Nrf2 dissociates from Keapl, enters the nucleus and activates the expression of the AREcontaining genes. According to more recent data, Keap1 is capable of entering the nucleus to mediate the proteasome-dependent degradation of the Nrf2 in the nucleus (Nguyen et al., 2009). 
The Neh6 domain is involved in Keap1-independent degradation of Nrf2. Glycogen synthase kinase 3 (GSK-3) phosphorylates a group of Ser residues located in the Neh6 domain, allowing the S-phase kinase-associated protein 1/cullin 1/F-box (SCF) protein complex (that contains the F-box protein $\beta$-transducin repeat-containing protein ( $\beta$-TRCP or SCF $(\beta$-TRCP)) to bind and catalyze the ubiquitination of Nrf2 in a manner similar to that of Keap1, leading to the proteasomal destruction of Nrf2 (Rada et al., 2011). Compared to Nrf2, Nrfl has several unique domains, highly conserved among Nrf1 homologues in other species, which are thought to confer Nrfl-specific functions. Among such domains is the endoplasmic reticulum- (ER)-targeting sequence, located in the N-terminal domain (NTD, Figure 1.3) of the protein.

The activity of Nrfl appears to be negatively controlled by the NTD, which directs Nrfl to the ER, where Nrfl is in its inactive state (Zhang et al., 2006; Zhang et al., 2009; Wang and Chan, 2007). In addition, Nrf1 is different from Nrf2 in that it contains an acidic/polar region (residues 125-155) within its acidic domain 1 (AD1). Immediately next to this region, Nrfl contains Neh2-1ke domain (Neh2L), containing Keap1-like binding motifs present in Nrf2. Later analysis revealed that, while the Neh2L region is involved in the Keap1 binding to Nrfl, it affects neither localization nor activity of Nrfl (Zhang et al., 2006). Further, Nrf1 possesses Asn/Ser/Thr-rich domain (NST) which contains seven Asn residues, at which Nrfl is putatively glycosylated in the ER. Finally, while both factors appear to have similar DNA-binding specificity and similar dimerization domain structure, it is the difference between the transactivation domains of Nrf1 and Nrf2 which is thought to give rise to some genes being preferentially controlled 
by either one of these factors (Ohtsuji et al., 2008). Such structural differences may enable Nrf1 and Nrf2 to recruit different co-activators, achieving specificity in their control of ARE-driven gene expression (Ohtsuji et al., 2008). 


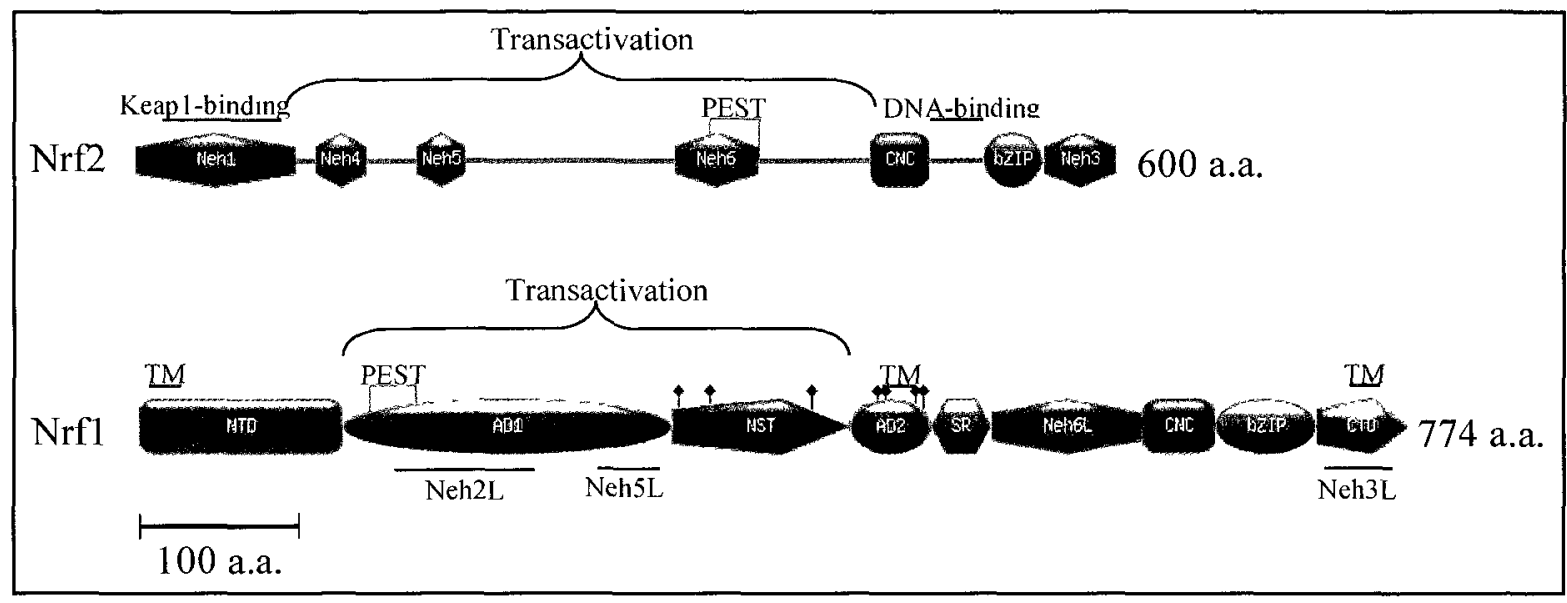

Figure 1.3. The Comparison Between the Nrf2 and Nrf1 Domains. The two factors have been named as "NF-E2-like" factors based on the presense of the the CNC domain. Nrf2-erythroid-derived CNC homology protein (ECH) homology (Neh) domains on Nrf2 and Neh-like (Neh2L, Neh2L and Neh3L) domains on Nrf1 are indicated. The Nrf1 domains are: NTD, N-terminal domain AD1, acidic domain 1, NST (Asn/Ser/Thr-rich region), $\mathrm{AD} 2$, acidic domain 2, SR (Ser repeat) domain and $\mathrm{CTD}, \mathrm{C}$-terminal domain. Both transcription factors contain CNC, cap'n'collar domain and bZIP, basic Leu zipper domains. TM indicates putative trans-ER membrane glycosylation sites (on NST and AD2 domain of Nrf1) are represented by sticks. Note that only Nrf1 possesses the NTD and both Nrf1 and Nrf2 contain a strong PEST sequence. Drawing of the domains and specific regions was performed to scale using the Prosite Mydomains feature of the ExPASy (http://prosite.expasy.org/mydomains). The word "domain" is used here to indicate specific amino acid regions rather than independent folding motifs. 
For example, while both $\mathrm{Nrf1}$ and Nrf2 appear to bind to the metallothionein 1 (mtl) promoter with equal affinity, Nrf2 was able to only weakly activate the $m t 1$ ARE-driven reporter vector in a co-transfection reporter assay. Similarly, Nrf2 stabilization by Keap1 knockdown in mice was ineffective at $m t 1$ induction, while other well-established $\mathrm{Nrf} 2$ target genes were downregulated in Keap1-deficient animals (Ohtsuji et al., 2008), suggesting that, despite the fact that both Nrfl and Nrf2 bind the $m t 1$ ARE, only Nrf1 can form necessary interactions with the coactivators, resulting in the transcription of this gene.

The uniqueness of Nrfl is also manifested in that, unlike Nrf2 or Nrf3, it is essential for embryonic development; $n r f l \%$ mice die at mid-late gestation, presumably due to anemia-induced hypoxia (Chan et al., 1998). Liver-specific knock out of Nrf1 resulted in the development of $n r f 1-/$ mice hepatic cancer with the phenotype reminiscent to nonalcoholic steatohepatitis, presumably due to the increased overexpression of the cytochrome P450 4A enzymes, catalyzing the oxidation of fatty acids and diminished ARE-driven gene expression (Xu et al., 2005). Similarly, hepatocytes isolated from the $n r f 1-/-$ animals contained higher number of lipid vesicles and exhibited greater proliferation of smooth ER (Ohtsuji et al., 2008; Xu et al., 2005). In contrast, $n r f 2-/-$ and $n r f 3-/-$ mice showed no obvious phenotype under unstressed conditions (Chan and Kan, 1999; Derjuga et al., 2005). The structural features of Nrfl, most notably, the presence of the NTD with a potential cholesterol recognition sequence

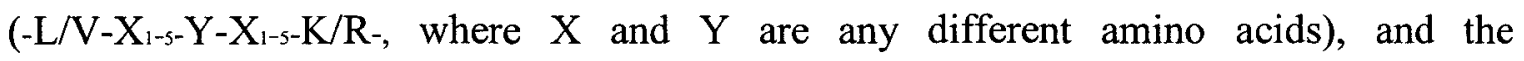
observation that Nrfl is localized to detergent-resistant lipid rafts of the ER (Zhang et al., 
2009), suggests that Nrfl is anchored to the ER and intramembrane proteolysis of Nrfl releases the active portion of the protein to travel to the nucleus and act as a transcription factor. This is similar to the regulation of another membrane-bound transcription factor, the Sterol Regulatory Element-binding Protein (SREBP). This, as well as the fact that $n r f 1-/-$ mice are embryonic lethal (nrf2-/- animals live to adult stage and are healthy under unstressed conditions), is consistent with the idea that Nrf1 has acquired distinct roles from other CNC-bZIP transcription factors during its molecular evolution.

Nrf1 localizes primarily to the ER (see Figure 1.4) as well as the nuclear envelope membrane (Zhang et al., 2009). The ER membrane-resident form of Nrf1 represents a low activity, glycosylated protein with an apparent molecular weight of $120 \mathrm{kDa}$ (p120), while the nuclear form (p95) is active, and unglycosylated (Zhang et al., 2007). The ER location of Nrf1 is thought to be suitable for the maintenance of the ER redox homeostasis (Zhang et al., 2009), perhaps by affecting the ER membrane lipid organization via its amphipathic, transmembrane $\alpha$-helices and participating in membrane-dependent biological events (Zhang and Hayes, 2010). Nrf1 may, for example, affect the rigidity of the ER membrane, acting as a diffusion barrier to membrane lipids (Zhang and Hayes, 2011). This ER homeostasis-maintaining function could not be attributed to any other ER-resident protein because the absence of Nrfl in mouse hepatocytes, isolated from the $N r f I-/-$ animals, contained higher number of lipid vesicles and exhibited greater proliferation of smooth ER (Xu et al., 2005). This suggested that $\mathrm{Nrfl}$ is required for the proper function of the ER. It has also been proposed that the localization of Nrfl within the ER determines the activity of this CNC 
factor and that the ER redox status and Nrfl glycosylation status could cause Nrfl to relocate from the ER to the nucleus (Zhang et al., 2009). As ER-resident Nrfl represents glycosylated $\mathrm{Nrfl}$ and nuclear-localized Nrfl is unglycosylated, it has been hypothesized that Nrfl deglycosylation could represent the main mechanism of its regulation (Zhang et al., 2009) and that Nrfl is activated by the proteasomal cleavage of its $\mathrm{N}$-terminus to remove the inhibitory NTD, producing smaller, more active forms of Nrf1 (Schultz et al., 2010). In addition, there is a 655 form of $\mathrm{Nrfl}$, presumably arising from $\mathrm{Nrfl}$ translation initiating at an internal start codon (Chan et al., 1993) although the possibility of the p65 arising as a result of a proteolytic cleavage can not be ruled out (Zhang et al., 2007). The p65 form has been shown to act as a dominant negative inhibitor of Nrf2-mediated, AREdriven luciferase activity (Wang et al., 2007). Apart from antagonistic competition of Nrf1 p65 (and, potentially, full-length Nrf1) with Nrf2 for the ARE binding site, the $n r f 2$ promoter contains two ARE sequences (Kwak et al., 2002), which may provide Nrf1 with yet another means of regulating $\mathrm{Nrf} 2$ expression. 
p120 Nrf1 (full-length protein, glycosylated), p95 Nrf1 (full-length, non/de-glycosylated)
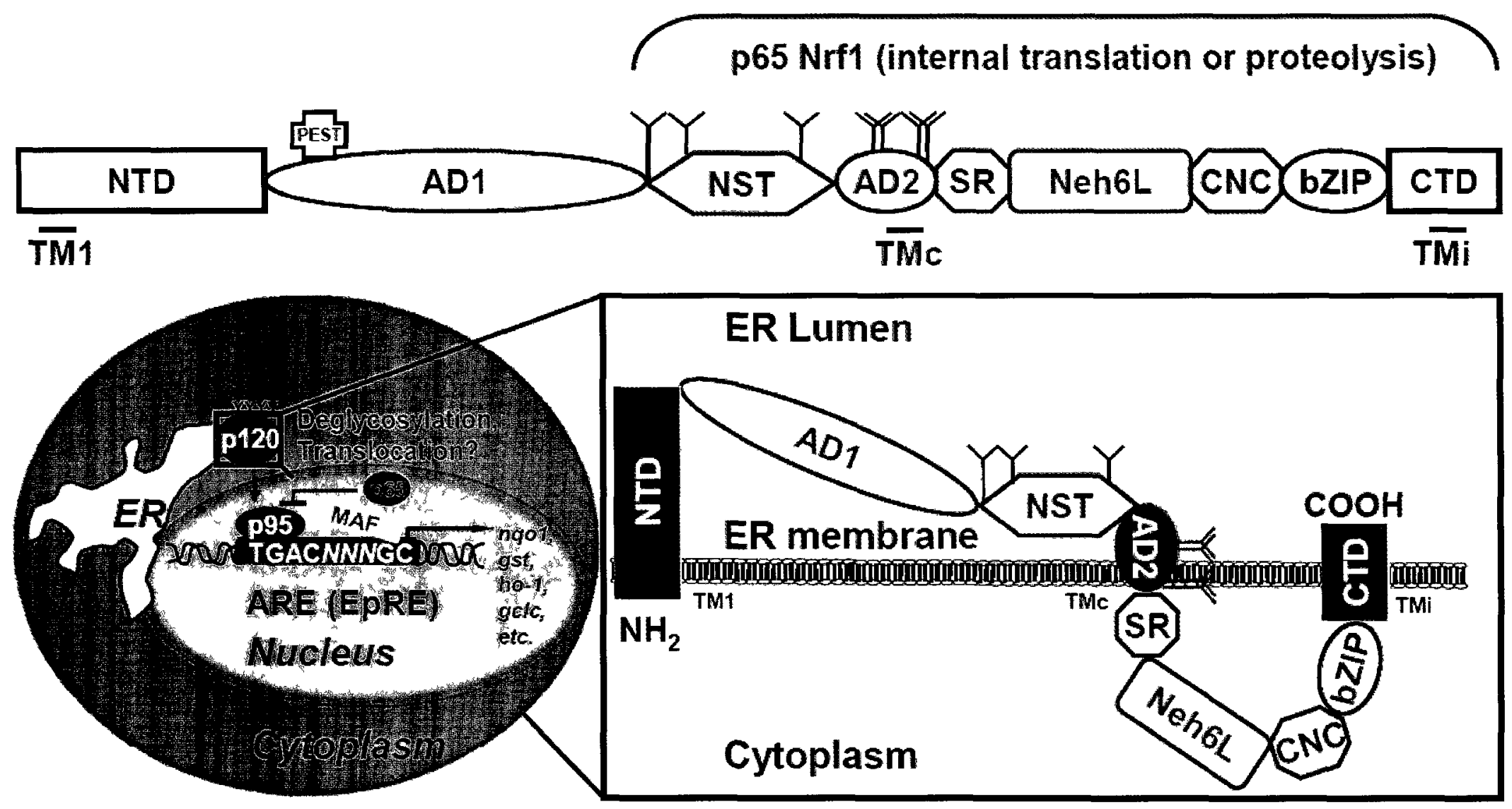
Figure 1.4. The Structural Domains in Human Nrf1 (hNrf1) and the Predicted Topology of Nrf1 Within the ER membrane. The domains (independent structural and homology regions) were identified using a multiple amino acid (aa) alignment with mouse $\mathrm{Nrfl}$ (mNrf1) aa sequence and domain designations as reported in (Zhang et al., 2006). The hNrf1 domains are: NTD, N-terminal domain (aa 1-124); AD1, acidic domain 1 (aa 125-324); NST, Asn/Ser/Thr-rich region (aa 325-432); AD2, acidic domain 2 (aa 433-482); SR, Ser repeat (aa 483-519); Neh6L, Neh6-like (aa 520-611); CNC, cap'n'collar (aa 612-655); bZIP, basic Leu zipper domain (aa 656-717); CTD, C-terminal domain (aa 718-772). The topology of mNrfl was predicted by Zhang and others (2009). TM1, TMi and TMc are putatitve trans-ER membrane regions. Nrfl is synthesized as an ER-targeted protein and, once inserted into the ER membrane via TM1, TMi and TMc, is glycosylated in the ER lumen; the Nrfl glycoprotein is referred to as p120. Following the translocation of the luminal part of the p120 into the nucleoplasm, it is unglycosylated to become active $\mathrm{p} 95 \mathrm{Nrf1}$, heterodimerizes with small Maf or c-Jun proteins and binds to the ARE (EpRE) to activate the expression of genes involved the antioxidant defense and phase II detoxification metabolism. In vitro, Nrfl deglycosylation is achieved by PNGase F. Internal translation or proteolysis by yet unidentified protease gives rise to p65 Nrfl, dominant negative repressor of the ARE-driven gene expression. Glycosylation sites are represented by "Y". Drawing of the domains and specific regions of Nrfl is to scale. 
In addition to the negative regulation of $\mathrm{Nrf} 2$ by p65 $\mathrm{Nrfl}$, reported by Wang and colleagues (2007), the conditional knock-out work with hepatocyte-specific Nrf1knockout mice showed that Nrf1 deficiency upregulated the Nrf2-target genes, such as gclc, nqol and ho-1, while this effect was abolished in Nrf2-lacking animals (Ohtsuji et al., 2008). It is plausible to hypothesize that the upregulation of the Nrf2-controlling genes in the livers of the Nrfl-deficient animals is due to the failure of structurally-different transactivation domains of Nrfl to form the necessary interactions with the co-activator proteins to activate the transcription of the Nrf2-target genes. Therefore, when the inhibitory effect of $\mathrm{Nrfl}$ is removed, more Nrf2-ARE interactions can take place, resulting in increased gene expression. Another possibility is that the lack of Nrf1 can create oxidative stress conditions, which would stimulate Nrf2-driven gene expression (Ohtsuji et al., 2008). Yet, another plausible scenario is the one in which a competition for the ARE binding takes place between the p65 Nrf1 and Nrf2 such that the knockout of $n r f 1$ removes the negative, inhibitory action of $\mathrm{Nrfl}$, leading to increased transcription of certain ARE-driven genes (Yamamoto, personal communication; Forman, personal communication). Such Nrf1-Nrf2 competition could be a useful way of turning down unnecessary transcription of antioxidant genes which, if left unrestrained, could lead to promoting the proliferation of precancerous cells and their survival (Wang et al., 2007). The present thesis examines, in greater detail, the functional interaction between $\mathrm{Nrfl}$ and Nrf2 factors, in addition to examining the relationship between different forms of $\mathrm{Nrfl}$, as well as the regulation of $\mathrm{Nrfl}$ 
by various conditions and post-translational modifications, are described in the Research Hypotheses section in greater detail.

\subsection{ROS, Nrf2 and Their Potential Involvement in Aging}

According to the oxidative stress theory of aging, the current version of the free radical theory of aging, proposed nearly 60 years ago (Harman, 1956), the use of oxygen by all aerobic cells creates ROS, damaging cellular macromolecules even under normal physiological conditions (Sohal and Weindruch, 1996). The accumulation of oxidative damage increases with age and is thought to be the major cause of senescence (Sohal an Weindruch, 1996). In support of this theory, multiple studies reported that aging organisms display increased oxidative damage (Bokov et al., 2004); however, the accumulated genetic evidence, aimed at altering the extent of oxidative damage in animals, suggests that oxidative damage plays a major role in aging only under suboptimal conditions and plays a very minor role in aging in unstressed environments (Salmon et al., 2009). Similarly, there is some evidence, suggesting that the susceptibility of animals to pro-oxidants, compounds augmenting oxidative stress within cells, increases with age (Gordon et al., 2010). At the systemic level, greater susceptibility of the elderly to environmental toxicants may be the consequence of the age-associated changes in the nervous system (Ginsberg et al., 2005) while at the cellular level, aging is associated with diminished expression of the antioxidant genes, orchestrated by $\mathrm{Nrf} 2$ (Suh et al., 2004; Shih and Yen, 2006). Interestingly, the proper function of the Nrf2- 
ARE pathway seems to be crucial for long-lived animals such that Nrf2 has been regarded as the "gatekeeper of species longevity" (Lewis et al., 2010). Paradoxically, aged animals display impaired $\mathrm{Nrf} 2$ function and reduced $\mathrm{Nrf} 2$ levels when its activity is most needed (Bohman and Sykiotis, 2010). Since Nrf2 is inducible by multiple prooxidants, nanoparticles generated by the vehicular traffic have a tendency to generate free radicals due to the presence of organic hydrocarbon and transition metals (Morgan et al., 2011; Xia et al., 2006), the treatment of young and aged mice with airborne particles was thought to be a suitable model for the study of the Nrf2-ARE pathway induction in vivo. Given the potentially negative regulation of Nrf2 by Nrfl, which has been discussed in greater detail above, we wanted to consider whether Nrfl activity and expression is altered by age and whether such an alteration can provide a clue to $\mathrm{Nrf} 2$ inactivation in aged animals.

\subsection{Bisphenol A, the Mechanism of Its Action in Biological Systems and Its Relation to Oxygen Metabolism and Antioxidant Enzymes}

Bisphenol A (BPA) is used in the production of polycarbonate plastics and epoxy resins for baby bottles, liners of canned food, medical equipment and consumer electronics (Benachours and Aris, 2009). Unfortunately, BPA-containing products can act as a source of the human exposure to BPA, especially under high temperature, exposure to sunlight, acidic or basic conditions (Kang et al., 2003). Indeed, over $90 \%$ of the U.S. population has detectable levels of BPA in urine with the chemical being most prevalent 
in children samples (Calafat et al. 2005). Although initially thought of as a weak estrogenic compound, it is now apparent that the potency of BPA to stimulate several cellular responses is comparable to estradiol (Rubin, 2011) and BPA is thought to act as an endocrine disruptor, interfering with normal pre- and post-natal development (O'Connor and Chapin, 2003). The developing liver of embryos and neonates has relatively weak capacity to metabolize BPA (Takahashi and Oishi, 2000). The latter fact probably explains the reason Canadian government banned the use of BPA-containing plastics in the production of baby food bottles in 2008. More specifically, BPA exposure was found to lead to unusual reproductive organ development (Lang et al. 2008), diabetes (Alonso-Magdalena et al. 2010) and decreased fetal survival and fetal body weight (Rubin et al. 2009).

BPA has the propensity to be metabolized to a catechol and then, subsequently, to an ortho-quinone and these two species can participate in the redox cycling (Kovacic, 2010). More specifically, the tendency to generate ROS is due to the highly reactive nature of the ortho-quinones that can donate electrons to molecular oxygen, producing superoxide, $\mathrm{O}_{2}^{-}$and other potentially damaging forms of ROS. Previous studies suggested that BPA treatment of cultured mammalian cells, in the order of $50-100 \mu \mathrm{M}$ BPA for 6-24 hours, increases the formation of ROS and the production of antioxidant enzymes, many of which are transcriptionally regulated by the ARE. However, despite increased expression, many antioxidant enzymes were found to be inactivated by BPA application. For instance, BPA treatment of cultured human Chang liver cells led to inactivation of antioxidant enzymes catalase and glutathione peroxidase (CAT and GPx, 
respectively, Oh and Lim, 2008). Both CAT and GPx are thought to be controlled by the ARE pathway (Itoh et al., 2005; Ohtsuji et al., 2008). Similar inactivation of GPx and CAT was seen in vivo in mice and rats during the provision of a BPA-rich diet to mice (Kabuto et al., 2003) and rats (Chitra et al., 2003). I hypothesized that the stimulatory effect of the BPA on ROS generation could be due to the impairment of the ARE-Nrf1/2 pathway and declined mRNA expression of the ARE-controlled antioxidant enzymes.

\subsection{Research Hypotheses}

Three main themes have been considered during the completion of this thesis. These pertain to the regulation of Nrfl by: i) the proteasome (and proteasome inhibitors) and hypoxia, ii) BPA, and iii) aging and airborne particles. The common theme in using these treatments and conditions is that they are all thought to be related to oxidative stress conditions and thus fall within the topic of "oxygen-dependent" regulation of Nrfl.

First, the proteasome regulates some rapidly-degraded transcription factors, such as Nrf2 and HIF- $\alpha$. In addition, the proteasome is responsible for destruction of aged, oxidized, misfolded or unfolded proteins and the impairment of proteasomal function can lead to augmented intracellular ROS production and cell death (Grim et al., 2011). Proteasome inhibition also stimulates the hypoxia- and oxidative stress-inducible gene expression through the stabilization of transcription factors HIF- $\alpha$ and Nrf2, respectively.

I hypothesized that Nrfl is regulated by the proteasome through rapid proteasomal degradation. I also hypothesized that hypoxia has an effect on the Nrf1-ARE pathway. In 
addition, I hypothesized that there could be several post-translational mechanisms of Nrfl regulation. To test these hypotheses, I used cultured mammalian cells in conjunction with the proteasomal inhibitors and hypoxic conditions and analyzed the expression of Nrfl and the function of Nrfl, overexpressed in the cells and subjected to different conditions using luciferase reporter assay.

Next, given the reported pro-oxidant activity of BPA in cultured cells, I hypothesized that BPA might lead to ROS generation by affecting the NrfI/2-ARE pathway-mediated expression of antioxidant genes. To test this hypothesis, I used BPA treatment of cultured human cells. ROS levels were measured using conventional fluorescence-based assays and the expression of the regulatory ( $\mathrm{Nrf1}$ and $\mathrm{Nrf2}$ ) and target (Nqo1 and HO-1) proteins was analyzed using immunoblotting and real-time quantitative RT-PCR. The function of Nrfl and Nrf2, overexpressed in the human cultured cells was measured upon BPA treatment using luciferase reporter assays.

Finally, I investigated the potential regulation of Nrfl by aging and particulate exposure using in vivo mouse models. I hypothesized that Nrfl can negatively regulate the transactivation activity of Nrf2 in vitro and in vivo and that Nrf1 may be differentially expressed in mousemice as a function of age. To test this hypothesis, I used luciferase reporter assays and overexpressed short (p65) and full-length Nrfl forms, in addition to $\mathrm{Nrf2}$, and followed the transactivation activity of $\mathrm{Nrf} 2$ in the presence of $\mathrm{Nrfl}$. In addition, I used the mouse models of airborne particle exposure since airborne particles are reactive centres with rich chemistry stemming from the presence of organic 
hydrocarbons and transition metals and analyzed and I analyzed Nrfl and Nrf2 protein expression and DNA binding functions using lung protein extracts. 
2 Chapter: Methods 


\subsection{Justification for the Use of Specific Cell Lines and Experimental Design}

COS7 and HEK293A are immortalized cells from the kidney of African green monkey and human embryos, respectively. These cells undergo transfection very easily and are routinely used for transcription factor studies. For example, Numazawa and co-workers used $\operatorname{COS} 7$ to study the activation of Nrf2 by atypical protein kinase C (2003). Since $\mathrm{Nrfl}$ is expressed ubiquitously (Chan et al., 1993), the use of a cell line in which some basal expression of Nrfl protein exist in addition to the expression of the FLAG-tagged Nrfl was considered appropriate to study the function of this transcription factor, which is a common strategy, reflected in other publications (e.g., Furukawa and Xiong, 2005). Simultaneous utilization of luciferase reporters with overexpressed transcription factors usually does not provide researchers with the same degree of confidence as when utilizing other experiments, as will be discussed in Chapter 6 (Figure 6.2); however, this approach still provides important clues to a regulation of transcription factor and such an approach is often employed by researchers in the field. This approach has been utilized to reveal the transactivation regions of $\mathrm{Nrf3}$ (Chénais et al., 2005). Hypoxic activation of Nrf1-FLAG, seen initially in COS7 cells, was confirmed using Nrf1-FLAG overexpression in the HEK293 cells. WFF 2002 cells are whole fetal fibroblasts derived from human, female, and non-cancerous fetuses. This cell line was used to confirm the data, obtained with proteasomal inhibition of $\mathrm{Nrfl}$, as seen in COS7 cells, in order to reveal if proteasomal regulation of $\mathrm{Nrfl}$ is a universal mechanism of Nrfl regulation, seen in different species. For cycloheximide (CHX) experiments, Nrfl p120 was first stabilized by proteasomal inhibition in $\operatorname{COS} 7$ cells and then the proteasome inhibitor- 
containing medium was removed and $\mathrm{CHX}$ was added to inhibit protein synthesis. The assumption here was that the increase in the proteasome-degraded proteins, achieved by proteasomal inhibition did not overwhelm the catalytic activities of the proteasome and that the rate of $\mathrm{Nrfl}$ degradation was not affected by pre-treatment with proteasomal inhibitor MG-132, which is known to inhibit the proteasome reversibly (Pajonk et al., 2005). Such as strategy for the determination of a half-life was employed for HIF-1 $\alpha$ in which HIF-1 $\alpha$ was first stabilized by pre-treatment with $10 \mu \mathrm{M}$ MG-132 for 15 min, followed by CHX exposure (Chachami et al., 2004).

Another important consideration taken into account was the toxicity of certain treatments, dependent upon the time and concentration of a condition or a compound exposure, provided to the cells. This has been addressed by using the time and concentrations previously reported in the literature as being non-cytotoxic (e.g., Nouhi et al., 2007) and the lack of cytotoxicity upon treatment with proteasome inhibitors has been confirmed using cell viability assays, including the methylthiazolyldiphenyltetrazolium bromide (MTT), trypan blue and cell sorting/GFP assays (data not shown).

The cellular context of the experiments was also considered in the interpretation of the data. For example, since the phosphatase and kinase inhibitors used were not Nrflspecific and were expected to affect other proteins, regulated by phosphorylation, it has been explicitly discussed (section 3.7 ) that the experimental outcomes could not provide any information, suggesting whether or not the phosphorylation status of Nrfl itself has been affected by the treatment and the possibility of the existence of other proteins, 
acting on Nrfl in a phosphorylation-dependent manner, has been mentioned. AAPH (2,2'-azobis(2-amidinopropane) dihydrochloride), a known peroxyl radical generator, is usually used at high millimolar $(10-100 \mathrm{mM})$ concentrations to achieve a steady, continuous rate of the radical generation (Niki, 1990) and has been used to study the oxidative inactivation of several enzymes, including catalase, alcohol dehydrogenase and horseradish peroxidase (reviewed in Chepelev et al., 2009) at these concentrations.

For the work presented in Chapter 4, the cell viability with BPA treatment was performed. The 24-hour time interval for treatment with several concentrations of BPA is common methodology in many toxicological studies that examine effects of specific chemicals published to date. For example, Li et al. (2005) used a single concentration of tert-butylhydroquinone (tBHQ) and a single time point ( 24 hours) to discover genes, whose expression was altered by the tert-hydroquinone treatment. Likewise, in this work, a known inducer of $\mathrm{Nrf2}$, tBHQ, ( $\mathrm{Li}$ et al, 2005), was used as a positive control for Nrf2 transactivation activity.

For Chapter 5, human bronchial epithelial (HBE1) cells were used as they retain the phenotype of normal bronchial epithelial cells and could, therefore, be a useful model of human lung cells (Yankaskas et al., 1993). Finally, mice, treated with airborne particles, were used for $\mathrm{Nrfl} / 2$ studies as the airborne particles used for the experiment were found to contain stable, carbon-centred radicals (Morgan et al., 2011). Lung was used as the tissue of choice as it was in direct contact with the inhaled particles. 


\subsection{Cell Culture and Treatments}

COS7 cells were grown in DMEM supplemented with $10 \%$ NCS and 3\% P/S/A (300 units $/ \mathrm{mL}$ penicillin $\mathrm{G}$, sodium salt, $300 \mu \mathrm{g} / \mathrm{mL}$ streptomycin sulfate and $0.75 \mu \mathrm{g} / \mathrm{mL}$ Fungizone ${ }^{\circledR}$ in $0.85 \%$ saline) in a humidified atmosphere with $5 \% \mathrm{CO}_{2}$ at $37^{\circ} \mathrm{C}$. HEK293A and WFF2002 cells were grown under the same conditions, but with different sera (10\% HS and 10\% FCS, respectively). Hypoxic conditions were achieved by setting $\mathrm{O}_{2}$ at $1 \%, \mathrm{CO}_{2}$ at $5 \%$ and the balance $\mathrm{N}_{2}$ in triple-gas incubators (Thermo Forma, Rockford, IL) or by incubating the cells with chemicals at the concentrations and times indicated in the figure legends.

\subsection{Immunoblotting and Co-immunoprecipitation}

Harvested cells were lysed in 50 to $100 \mu \mathrm{L}$ of cell lysis buffer (20 mM HEPES pH 7.9), $420 \mathrm{mM} \mathrm{NaCl}, 1.5 \mathrm{mM} \mathrm{MgCl}_{2}, 0.2 \mathrm{mM}$ EDTA and $25 \%$ glycerol, $1 \mathrm{mM}$ DTT and 0.5 mM PMSF) and an equal amount of total protein (determined using BioRad protein assay and diluted 1:1 with $2 \times$ Laemmli loading buffer) was loaded on a 10\% SDS-PAGE, run at $120 \mathrm{~V}$ for 1.5 to 2 hours and transferred onto Immobilon PVDF membrane (Millipore, Bedford, MA) overnight at $4^{\circ} \mathrm{C}$. Membranes were probed with $5 \%$ milk in Tris-Buffered Saline, Tween-20 (TBST) for 1 hour. Western blot analysis for Nrf1 was performed using mouse anti-human Nrfl, HO-1 and Nqo1 antibody (1:1,000 dilution) and rabbit antihuman Nrf2 (Santa Cruz Biotechnology and horseradish peroxidase-labelled goat antimouse or goat anti-rabbit IgG secondary antibody (1:2,000 dilution; DAKOCytomation, 
Mississauga, ON). FLAG- tagged Nrfl was visualized using horseradish peroxidase (HRP)-conjugated mouse anti-FLAG M2 antibodies (1:4,000 dilution; Sigma-Aldrich). As a loading control, membranes were probed with mouse anti-human $\beta$-tubulin (1:4,000 dilution, Developmental Studies Hybridoma Bank, Iowa City, IA) or stained with Ponceau S red stain. Blots were developed by enhanced chemiluminescence substrate (Millipore, Bedford, MA) and Kodak X-Omat blue film (Perkin-Elmer, Waltham, MA). Film was scanned using a CanoScan LIDE 80 scanner (Canon, Lake Success, NY) and band densitometry was measured using AlphaEaseFC software, version 3.1.2 (Alpha Innotech/Cell Biosciences, Santa Clara, CA). For co-IP, the protein complexes were immunoprecipitated from $125 \mu \mathrm{g}$ total lysate protein with $20 \mu \mathrm{L}$ Protein A/G-Sepharose beads and $2 \mu \mathrm{g}$ anti-Nrf1 antibody. The immunoprecipitated complexes were subjected immunobloting using anti-ubiquitin antibody.

\subsection{Cloning of Nrf1-FLAG}

Human Nrf1 gene from mammalian gene collection (accession number BC010623) was inserted into a modified pCR3.1 mammalian expression vector using NdeI and EcoRI and then introduced into CMV-5a-FLAG vector (Sigma-Aldrich) using EcoRI and Kpn1 restriction enzymes to create $\mathrm{N}$ - and C-terminally FLAG-tagged Nrfl, respectively.

\subsection{Transient Transfections and Luciferase Reporter Assays}


A luciferase reporter plasmid containing three AREs from chicken $\beta$-globin enhancer $(3 \mathrm{x}$ ARE-luciferase) was a kind gift from Dr. Masayuki Yamamoto (Tohuku University) and has been described elsewhere (Igarashi et al., 1994). N-terminally myc-tagged (pCDNA3-myc3-Nrf2) and N-terminally FLAG-tagged Nrf2 (pPROEX-HTc-Flag3Nrf2) were obtained from Addgene (Addgene plasmids 21555 and 21553 Cambridge, MA) and has been described previously (Furukawa and Xiong, 2005). Cells were seeded in 6-cm or 6-well plates at a density of 140,000 cells $/ \mathrm{mL}$ and approximately 24 hours later were transiently transfected with Lipofectamine 2000 as per the manufacturer's protocol (upon becoming at least $70 \%$ confluent). For a $6-\mathrm{cm}$ plate, $0.2 \mu \mathrm{g}$ of $\beta$ galactosidase, $8.0 \mu \mathrm{g}$ of $3 \mathrm{x}$ ARE-luciferase and $2.4 \mu \mathrm{g}$ of Nrf1-FLAG, myc-Nrf2 or pCR3.1 (empty vector) plasmid DNA, were used. Twenty-four or forty-two hours later, cells were treated for 24 or 6 hours, respectively, and harvested such that the total amount of time after transfection was $48 \mathrm{~h}$. A firefly luciferase reporter gene assay was performed to measure ARE-driven transcriptional activity. Cells were lysed in 25 to 50 $\mu \mathrm{L}$ of lysis buffer ( $25 \mathrm{mM}$ glycylglycine (pH 7.8), $1 \%$ Triton X-100, $15 \mathrm{mM} \mathrm{KH}_{2} \mathrm{PO}_{4}, 15$ $\mathrm{mM} \mathrm{MgSO}_{4}, 4 \mathrm{mM}$ EDTA, $1 \mathrm{mM}$ DTT and a complete protease inhibitor cocktail tablet (Roche, Mannheim, Germany)). Approximately 4 to $12 \mu \mathrm{L}$ of supernatant was added to $76 \mu \mathrm{L}$ of luciferase assay buffer ( $2 \mathrm{mM}$ ATP in lysis buffer) and luciferase activity was assayed on a FLUOstar OPTIMA (BMG LABTECH, Offenburg, Germany) luminescence microplate reader. The reaction was initiated by the injection of $50 \mu \mathrm{L}$ of $200 \mu \mathrm{M}$ luciferin solution. Luciferase assay values were normalized to $\beta$-galactosidase assay results (a measure of transfection efficiency). For $\beta$-galactosidase assay, cell lysates were incubated in $85 \mu \mathrm{L}$ of $0.2 \mathrm{mg} / \mathrm{mL}$ of $\mathrm{CPRG}$ in $60 \mathrm{mM} \mathrm{Na}_{2} \mathrm{HPO}_{4}$, (pH 8.0 ), $10 \mathrm{mM}$ 
$\mathrm{KCl}, 1 \mathrm{mM} \mathrm{MgCl}$, and $1 \mathrm{mM} \mathrm{DTT}$, for 5 to 10 minutes, and monitored at $580 \mathrm{~nm}$. Transfection experiments were reproduced at least three times and are presented as means \pm S.E.M.

\subsection{Electrophoretic Mobility Gel Shift Assays}

Cells were lysed as described above and 7.5 - $20 \mu \mathrm{g}$ nuclear or total protein was reacted with a biotinylated dsDNA ARE probe of the human gclm promoter (Kwong et al., 1998) and was subjected to non-denaturing electrophoresis according to the manufacturer's instructions (Panomics, Fremont, CA). Protein-DNA complexes were transferred onto an Amersham Hybond- $\mathrm{N}^{+}$membrane (GE Healthcare, Buckinghamshire, UK) and visualized using streptavidin-HRP and chemiluminescence as described above for Western blots. The identity of the ARE-bound complex was investigated using $2 \mu \mathrm{g}$ nonspecific (pre-immune serum with immunoglobulin $G(\operatorname{IgG})$ or actin) antibodies per reaction for immunodepletion (Dickinson et al., 2003). In this case, the protein extract is incubated with a given antibody for 2 hours at room temperature prior to the addition of the probe. To control for specific binding to the ARE probe, an excess of cold (unlabelled probe) provided by the manufacturer at an unspecified concentration (Panomics) was employed.

\subsection{Computational and Bioinformatics Analyses}


To compute the gas-phase bond dissociation enthalpies of the $\mathrm{O}-\mathrm{H}$ bonds in BPA, the medium level method 2 (MLM2; Wright et al., 2001) was used. Briefly, the molecular geometries were first approximated using lowest energy conformer generation procedure at the Austin Model 1 (AM1) level. The lowest energy conformer geometry was then used as a starting point for a geometry optimization procedure at the density functional theory (DFT) level with a Becke, Three-Parameter, Lee-Yang-Parr (B3LYP) functional and a $6-31 \mathrm{G}(\mathrm{d})$ basis set. The optimized geometry was used to compute the frequencies, at the same level of theory, with a correction factor of 0.9806 , at $298.15^{\circ} \mathrm{K}$ temperature and 1 atm. pressure, in order to obtain the enthalpic correction factors for each compound. The final single-point energy of each compound was computed using the B3LYP functional and a $6-311+\mathrm{G}(2 \mathrm{~d}, 2 \mathrm{p})$ basis set. The overall gas-phase enthalpy of formation was computed as a sum of the enthalpic correction factor computed in the frequency calculation step and the final single-point energy. The procedure was identical for the closed-shell and open-shell (e.g. radicals) compounds, with the exception that the latter had a single-point energy computed with the open-shell (RO)B3LYP functional. The enthalpy of formation of the hydrogen atom was -0.5 hartree. In order to compute the Bond Dissociation Energy (BDE) for the transformation RO-H $\rightarrow \mathrm{RO} \cdot+\mathrm{H} \cdot$, the enthalpies of formation of reactants were subtracted from those of products.

ClustalW tool (Larking et al., 2007) was used for multiple amino acid alignment (http://www.ebi.ac.uk/Tools/clustalw2/index.html). Nrfl PEST domain was identified using PESTfind algorithm that was previously located at https://emb1.bcc.univie.ac.at/toolbox/pestfind/pestfind-analysis-webtool.htm. This tool 
identifies the amino acid regions rich in Pro, Glu, Ser and Thr, compares them to known, short-lived proteins with PEST sequences and provides an index, indicative of the strength of a given putative PEST sequence. The prediction of phosphorylation sites was performed using NetPhos 2.0 server available at http://www.cbs.dtu.dk/services/NetPhos/ (Bloom et al., 1999). This tool uses neural networks and defined sequence logos of phosphorylation sites of Tyr, Ser and Thr, derived from the experimentally-confirmed phosphorylation sites. The prediction of kinase-specific phorphorylation sites (Bloom et al., 2004) was performed using http://www.cbs.dtu.dk/services/NetPhosK/. The underlying principles of this method are very similar to NetPhos 2.0 and rely on neural network-assisted analysis of a given amino acid sequence by comparing it to the experimentally-derived, site-specific preferences for the phosphorylation of several wellstudied kinases, including protein kinase $\mathrm{C}(\mathrm{PKC})$. The phosphorylation prediction tools were used to predict the potential phosphorylation sites and the effect of the intracellular phosphorylation status on the Nrfl activity was assessed by further experiments.

\subsection{Cell Viability (MTT) Assay}

The principle behind this assay is the oxidation of yellow methylthiazolyldiphenyltetrazolium bromide (MTT) by mitochondrial NADH dehydrogenase to produce a purple formazan product, whose formation can be followed spectrophotometrically. Cells were seeded at a density of 50,000 cells/mL and grown for $48 \mathrm{~h}$ and treated with the indicated BPA concentrations for $24 \mathrm{~h}$. MTT was then added $(0.5 \mathrm{mg} / \mathrm{mL})$ for $2 \mathrm{~h}$ and the absorbance was read at $570 \mathrm{~nm}$ with correction at $630 \mathrm{~nm}$. 


\subsection{ROS, Glutathione and Carbonyl Assays}

Intracellular ROS were detected using the Image-iT LIVE Green Reactive Oxygen Species Kit (Molecular Probes). Cells were grown for $48 \mathrm{~h}$ as described above, washed in PBS, incubated in $10 \mu \mathrm{M}$ of 5-(and-6)-carboxy-2',7'-dichlorodihydrofluorescein diacetate (carboxy- $\mathrm{H}_{2}$ DCFDA; an intracellular ROS probe) for $30 \mathrm{~min}$, washed in PBS and treated with BPA for $24 \mathrm{~h}$ using phenol red-free medium. AAPH, a generator of peroxyl and alkoxyl radicals (Niki, 1990), served as a positive control. Free and total reduced glutathione (GSH) was measured using the DetectX Glutathione Fluorescent Detection Kit (Arbor Assays). Butathione sulfoxamine (BSO), an inhibitor of GSH synthesis (Yim et al., 1994), served as a positive control. The generation of carbonyl groups, as a marker of oxidative damage to proteins (Chevion et al., 2000), was assessed using the OxyBlot Protein Oxidation Detection Kit (Millipore) according to the manufacturer's protocol using $7.5 \mu \mathrm{g}$ of total cellular protein per sample.

\subsection{Total RNA Isolation and Real-time Quantitative PCR (real-time qPCR)}

Total RNA from frozen cell pellets was extracted using the RNeasy Mini Kit (Qiagen). Procedures for cDNA synthesis and real-time $\mathrm{qPCR}$ were as previously described (Chen et al., 2009). Briefly, $1 \mu \mathrm{g}$ total RNA was reverse-transcribed to synthesize cDNA with the Retroscript Kit (Applied Biosystems/Ambion). Real-time qPCR was performed on Mx4000 Multiplex Quantitative PCR System using TaqMan 
Gene Expression Assays (Applied Biosystems; see Table 4.1 for assay information). Glyceraldehyde-3-phosphate dehydrogenase (GAPDH) expression was unaffected by BPA treatments (data not shown) and was considered a valid housekeeping gene. The fold change in expression for the gene-of-interest relative to GAPDH (gene-ofinterest/GAPDH) was calculated and compared to the control group (set as 1.0).

\subsection{Nanoparticle Production, Mouse Exposure and Tissue Processing}

Nanoparticle (also referred to as "particulate matter" or PM) collection and mouse treatment have been described previously (Morgan et al., 2011). Briefly, nanoparticles were collected with a High-Volume Ultrafine Particle (HVUP) Sampler (Misra et al., 2002) at $400 \mathrm{~L} / \mathrm{min}$ in Los Angeles City near the CA-110 Freeway. This is considered to be the mix of fresh ambient particles mostly from vehicular traffic nearby this freeway (Ning et al. 2007). The nanoparticles with diameter less than $200 \mathrm{~nm}$ were collected on pre-treated Teflon filters $(20 \times 25.4 \mathrm{~cm}$, PTFE, $2 \mu \mathrm{m}$ pore; Pall Life Sciences, Covina, CA). The collected nanoparticles were transferred into aqueous suspension by $30 \mathrm{~min}$ soaking of nanoparticle-loaded filters in Milli-Q deionized water (resistivity 18.2 mega $\Omega$; total organic compounds $<10 \mathrm{ppb}$; particle-free; bacteria levels $<1 \mathrm{CFU} / \mathrm{ml}$; endotoxinfree glass vials), followed by $5 \mathrm{~min}$. vortexing and $30 \mathrm{~min}$. sonication. Aqueous nanoparticle suspensions were pooled and frozen as a stock at $-20^{\circ} \mathrm{C}$, which is chemicaly stable for at least 3 months ( $\mathrm{Li}$ et al. 2003). The nanoparticle suspensions were reaerosolized by a VORTRAN nebulizer using compressed particle-free filtered air as previously described (Morgan et al., 2011). 
C57BL/6 male mice ( 3 month- and 18 month-old) were maintained under standard conditions with ad libitum Purina Lab Chow (Rancho Cucamonga, CA) and sterile water. Just before the exposure to particles, mice were transferred from home cages to exposure chambers, which provided free movement. Temperature and airflow parameters were controlled for adequate ventilation and minimization of the build-up of animal-generated contaminants (skin dander; $\mathrm{CO}_{2}, \mathrm{NH}_{3}$ ). Re-aerosolized nanoparticles or ambient air (control) was delivered to the sealed exposure chambers for $5 \mathrm{hr} / \mathrm{day}, 3$ days/week, for 10 weeks. Mice had normal weight and showed no signs of respiratory distress. After isoflurane anesthesia, mice were euthanized and the tissue was collected and stored at $-80^{\circ} \mathrm{C}$ until processing. All rodents were treated humanely, with regard for alleviation of suffering and following the procedures approved by the USC Institutional Animal Care and Use Committee. 
For protein extraction, approximately $30 \mathrm{mg}$ lung tissue was ground in glass homogenizer using $100 \mu \mathrm{L}$ of ice-cold CER I reagent from NE-PER Nuclear and Cytoplasmic Extraction Kit (Thermo Fisher Scientific, Rockford, IL), supplemented with protease and phosphatase inhibitors to prepare cytosolic fraction according to the manufacturer's protocol. For nuclear extraction, $50 \mu \mathrm{L}$ of NER reagent from the same kit, supplemented with phosphatase and protease inhibitors, was used, using the manufacturer's instructions. Protein determination was carried out using Bradford assay (BioRad, Hercules, CA).

\subsection{Oxygen Radical Absorbance Capacity (ORAC) Assay}

The antioxidant activity of lung cytoplasmic extracts was measured using the ORAC assay as descrubed previously (Cao et al., 1993). Three micrograms of cytoplasmic protein was used per well and the assay was run in triplicate. Trolox (6-hydroxy-2,5,7,8tetramethylchroman-2-carboxylic acid, a water-soluble vitamin $\mathrm{E}$ analogue), was used to establish the reference range. Peroxyl and alkoxyl radicals were generated using AAPH (2,2'-azobis (2-amidinopropane) dihydrochloride; final concentration $0.158 \mathrm{M})$, prepared fresh for each run. Fluorescein (final concentration $0.114 \mu \mathrm{M}$ ) was used as a fluorescence probe and rutin (final concentration $5.0 \mu \mathrm{M}$ ) was used as a positive control. Fluorescence was measured at an excitation and emission wavelengths of 360 and $520 \mathrm{~nm}$, respectively, using an automated plate reader (FLx800 Fluorescence Microplate Reader, BioTek) with 96-well plates. Analyses were conducted in $70 \mathrm{mM}$ potassium phosphate buffer $\mathrm{pH} 7.4$ at $37^{\circ} \mathrm{C}$. ORAC values were reported as $\mu \mathrm{mol}$ of Trolox equivalents ( $\mu \mathrm{mol}$ 
TE) that produces the same area under the curve as one microgram of total protein ( $\mu \mathrm{mol}$ $\mathrm{TE} / \mu \mathrm{g}$ total protein).

\subsection{Statistical Analyses}

Data are presented as means \pm S.E.M. of at least three independent experiments. The results were considered statistically significant at $\mathrm{p}<0.05$ for the Student's paired t-test unless otherwise indicated. 


\section{Chapter: Oxygen-dependent and Proteasomal Regulation of}

Nrf1 


\subsection{Proteasomal Inhibition Stabilizes p120 Nrf1 and Ubiquitinated Nrf1}

The regulation of the protein abundance through the degradation of misfolded and oxidatively damaged proteins is an important function of multi-subunit protease known as the proteasome. The $26 \mathrm{~S}$ proteasome is the most common of these. It consists of: a) the $20 \mathrm{~S}$ core with trypsin-, chymotrypsin- and peptidyl-glutamyl peptide-hydrolyzing proteolytic activities and b) the $19 \mathrm{~S}$ regulatory particle, containing the ATPase activity required for unfolding of the protein substrates prior to their degradation by the $20 \mathrm{~S}$ core. The attachement of ubiquitin, a short 76 amino acid protein, serves as the tag recognized by the $19 \mathrm{~S}$ regulatory particle. It is estimated that about $80 \%$ of cellular proteins are degraded by the ubiquitin-proteasome system (Crawford et al., 2011). It appears that not only misfolded or damaged proteins are the substrates of the proteasome, but also a number or regulatory proteins, including transcription factors. For instance, the key transcription factors, involved in hypoxia, inflammation and oxidative stress signalling cascades, including HIF- $\alpha$, IאB and Nrf2 respectively, are rapidly degraded by the proteasome (Taguchi et al., 2011). In other words, under unstressed conditions, the function of these factors is not required and, therefore, is repressed by continuous proteasomal degradation. Under an appropriate stimulus, however, the proteasomal inhibition of these factors is impaired and this de-repression from rapid proteasomal degradation through increased stability allows the cell to mediate appropriate responses through altered gene expression. Closely-related to Nrfl CNC-bZIP factors Nrf2 and Nrf3 have been shown to be controlled by keeping their intracellular levels low under homeostatic conditions through proteasomal degradation (Nguyen et al., 2003; Nouhi et 
al., 2007). I hypothesized that NrfI is also regulated by the proteasome through rapid proteasomal degradation. To test if $\mathrm{Nrfl}$ is also regulated via proteasomal degradation, I analyzed its protein expression in the presence of proteasomal inhibitors clastolactacystin- $\beta$-lactone (lactacystin) and MG-132. Proteasomal inhibition with both inhibitors greatly enhanced the expression of the inactive, ER-bound glycosylated form of Nrfl, p120 (Figure 3.1). Interestingly, while the density of the p120 band increased significantly (about 140\% compared to controls) during MG-132 treatment, the intensity of the band, corresponding to the active, nuclear nonglycosylated p95 Nrf1 form decreased (data not shown). This could imply that higher levels of p120 could arise, at least in part, due to increased glycosylation of $\mathrm{p} 95$, in response to proteasomal inhibition. However, the decrease in the p95 Nrfl seen during the MG-132 treatment was very minimal (approximately, 20\%) and, while such a decrease may be statisticallysignificant, this could be biologically-irrelevant. Also, this was not seen for lactacystin as the p95 expression during this treatment was unaltered, indicating that the stabilization of p120 may proceed independently of p95 Nrfl. This, apparently independent regulation of p95 and p120 was also confirmed by the treatment of $\operatorname{COS} 7$ cells with the oxidative stressor AAPH, which acts by generating steady-state levels of peroxyl and alkoxyl radicals and is employed at high millimolar concentrations (Niki, 1990). The danger of using such a high concentration of a xenobiotic compound is that it can lead to cell death through apoptosis, providing confounding results. During the AAPH treatment, it was assumed that, in the case of cell death, lowered tubulin signal will be observed, which was not seen. In addition, no detrimental effect of the AAPH treatment was observed using cells overexpressing $\beta$-galactosidase as judged by the $\beta$-galactosidase assay (data 
not presented). Upon establishing that AAPH, a known oxidative stressor, increased the Nrfl binding to the ARE (Figure 3.2A and B), we observed the accumulation of p95 form without any apparent effect on the p120, illustrating the possibility of p95 "de novo" accumulation (see Figure 3.2C). Alternatively, there may be some time lag between the accumulation of p120 and $\mathrm{p} 95$ forms, given the time required for $\mathrm{p} 120$ deglycosylation conversion to $\mathrm{p} 95$. 
Figure 3.1. Nrf1 is Stablized by Proteasomal Inhibition in COS7 cells. Cells were treated with $10 \mu \mathrm{M}$ lactacystin (A) or $10 \mu \mathrm{M}$ MG-132 (B) for six hours, lysed, resolved by SDS-PAGE and Western blots were performed with anti-Nrfl antibody as described in the Methods section (Chapter 2). DMSO (the solvent used for lactacystin and MG-132) was used for controls. The positions of p95 and p120 Nrfl, as well as the loading control $\beta$-tubulin, is shown with arrows and the presense or absence of a proteasomal inhibitor treatment is indicated by + or - , respectively. 


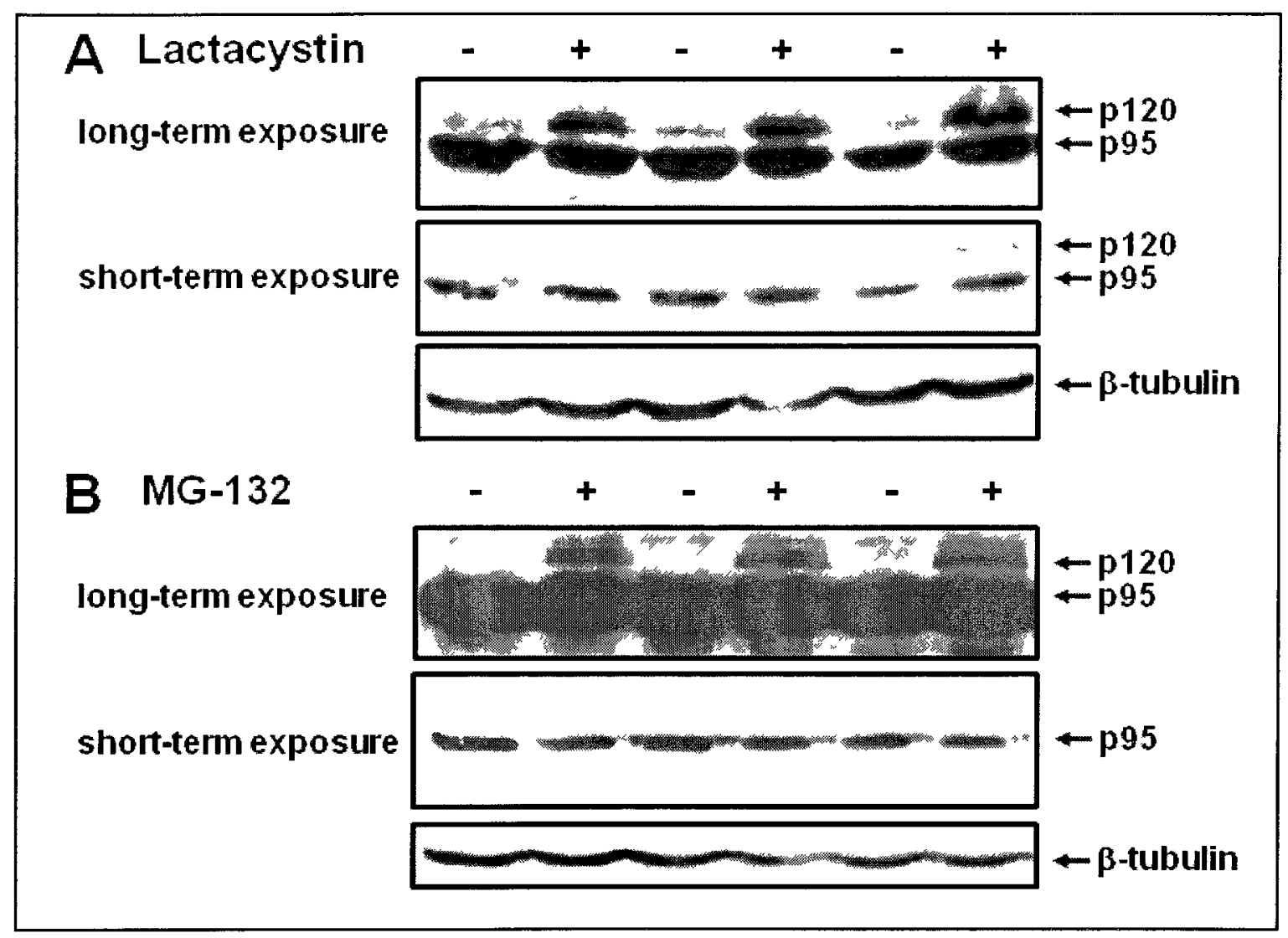


Figure 3.2. Oxidative Stressor AAPH Induces Nrf1 DNA Binding and Stabilizes p95 Nrf1 Form Independently of p120. (A) The Nrf1-specific band on an EMSA format was determined by including $20 \mu \mathrm{g}$ of the COS7 cell lysate with: 1) no antibody; 2) antiactin antibody; 3) anti-Nrfl antibody; 4) anti-Nrfl antibody, no lysate; and 5) neither lysate nor antibody (probe only). The experiment was performed twice with the same outcome. (B) COS7 cells were treated with 80 mM AAPH for 6 hours (Niki, 1990; Terao and Niki, 1986) and the lysates were subjected to EMSA and Western blotting (C). The positions of AAPH-inducible Nrfl-specific bands are shown with arrows in (A) and (B). In (C), both short- and long-time exposure of bands are shown for better clarity. 


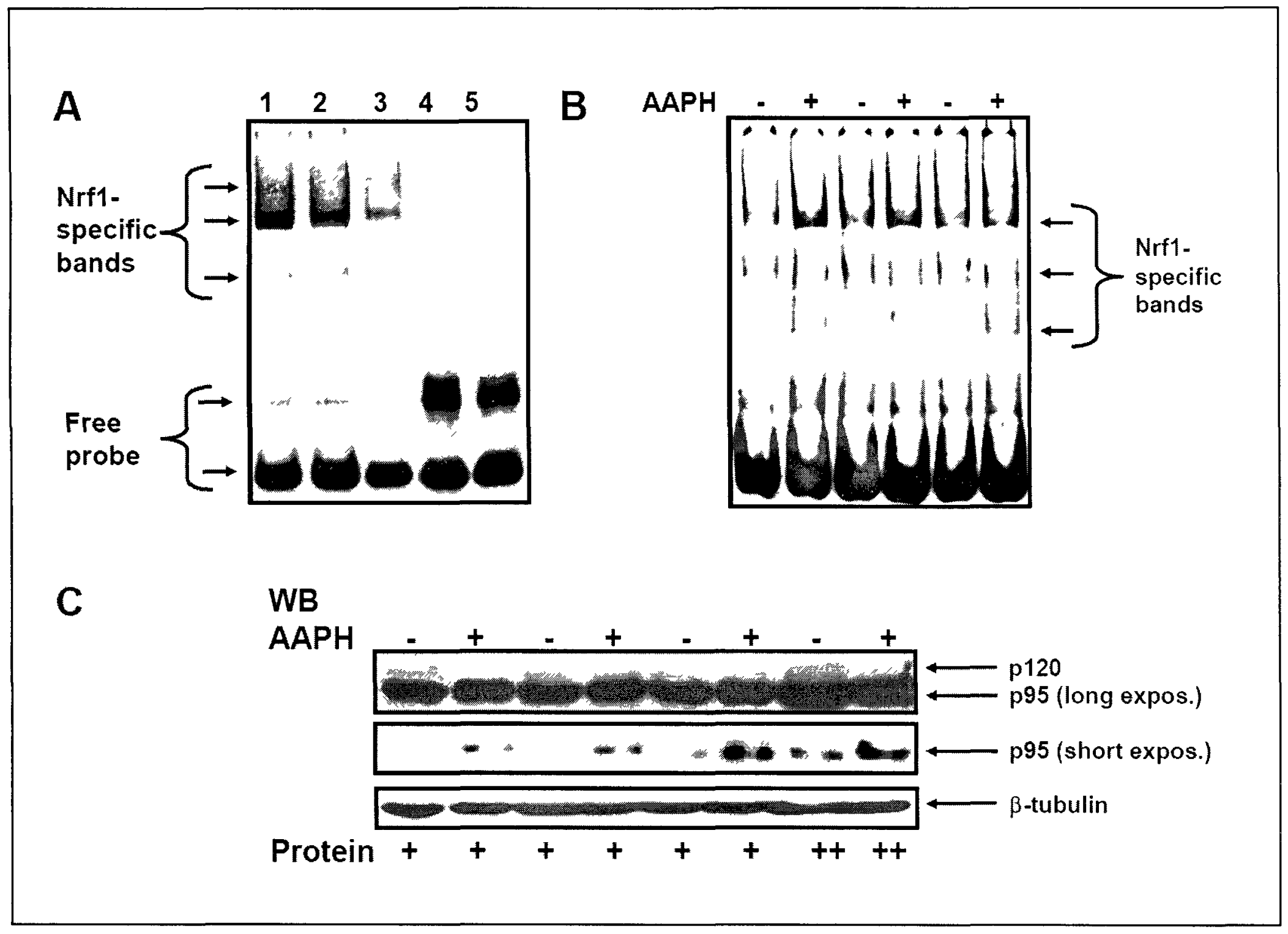




\subsection{Nrf1 Contains a PEST Motif and is Degraded by the Proteasome}

Nrf2 and Nrf3 have half-lives $\left(t_{1 / 2}\right)$ of approximately 15 and 30 minutes, respectively (Nguyen et al., 2003; Nouhi et al., 2007) and contain two strong putative PEST sequences (Nouhi et al., 2007; Steward et al., 2003). PEST sequences are rich in Pro, Glu, Thr and Ser and are found frequently in rapidly degraded proteins; the Ser and Thr residues are potential phosphorylation sites (Li et al., 2005). The PESTfind analysis tool (http://www.at.embnet.org/toolbox/pestfind/) was used to search for any putative PEST sequence in Nrfl and found that Nrfl has a strong PEST motif (amino acids 141-169) in its NTD (see Figure 1.4 for Nrfl domains). To test whether Nrfl is indeed subject to rapid degradation, as suggested by the presence of strong PEST motif, we applied CHX and analyzed Nrfl protein degradation by immunoblotting when protein synthesis was inhibited (Figure 3.3). A plot of band density versus time (Figure 3.3B) showed that the $\mathrm{t}_{1 / 2}$ of $\mathrm{p} 95$ is approximately $5 \pm 1$ hours in COS7 cells. Compared to transcription factors Nrf2 and Nrf3, Nrf1 is more than 5 to 10 times more stable, but the Nrf proteins are less stable than other proteins that have $t_{1 / 2}$ between 16 (lysozyme) and 210 (phosphoglycerate kinase) hours (Rogers et al., 1986). Compared to these proteins, Nrfl can be regarded as a relatively short-lived protein; however, amongst the transcription factors, it has a relatively long half-life, suggesting that Nrfl activity may be regulated by other mechanisms, such as post-translational modifications. Next, we found that anti-Nrfl antibody was able to capture significantly higher levels of ubiquitinated Nrfl, under MG132 treatment, compared to the DMSO control, in co-immunoprecipitation experiments, 
as revealed by immunoprecipitation using anti-Nrfl antibody followed by immunoblotting with anti-ubiquitin antibody (Figure 3.3C). 
Figure 3.3 Nrf1 Undergoes Ubiquitination and Proteasomal Degradation. (A) COS7 cells were treated with $12 \mu \mathrm{M}$ MG-132, a reversible proteasome inhibitor (Pajonk et al., 2005), for five hours to stabilize p120, the medium was replaced by fresh medium containing $100 \mu \mathrm{g} / \mathrm{mL}$ of CHX and the cells were harvested at different time intervals (08 hours) and subjected to immunoblotting. (B) The graphical determination of Nrfl p95 half-life $\left(\mathrm{t}_{1 / 2}\right)$ using at least three independent experiments \pm S.E.M. is shown. (C) Immunoprecipitation was performed using anti-Nrfl antibody as described above in the Methods section on the lysates of COS7 cells, pre-treated with $10 \mu \mathrm{M}$ MG-132 or DMSO for six hours and the ubiquitinated Nrfl was visualized using immunoblotting with antiubiquitin antibody. The results of two independent experiments are shown. 


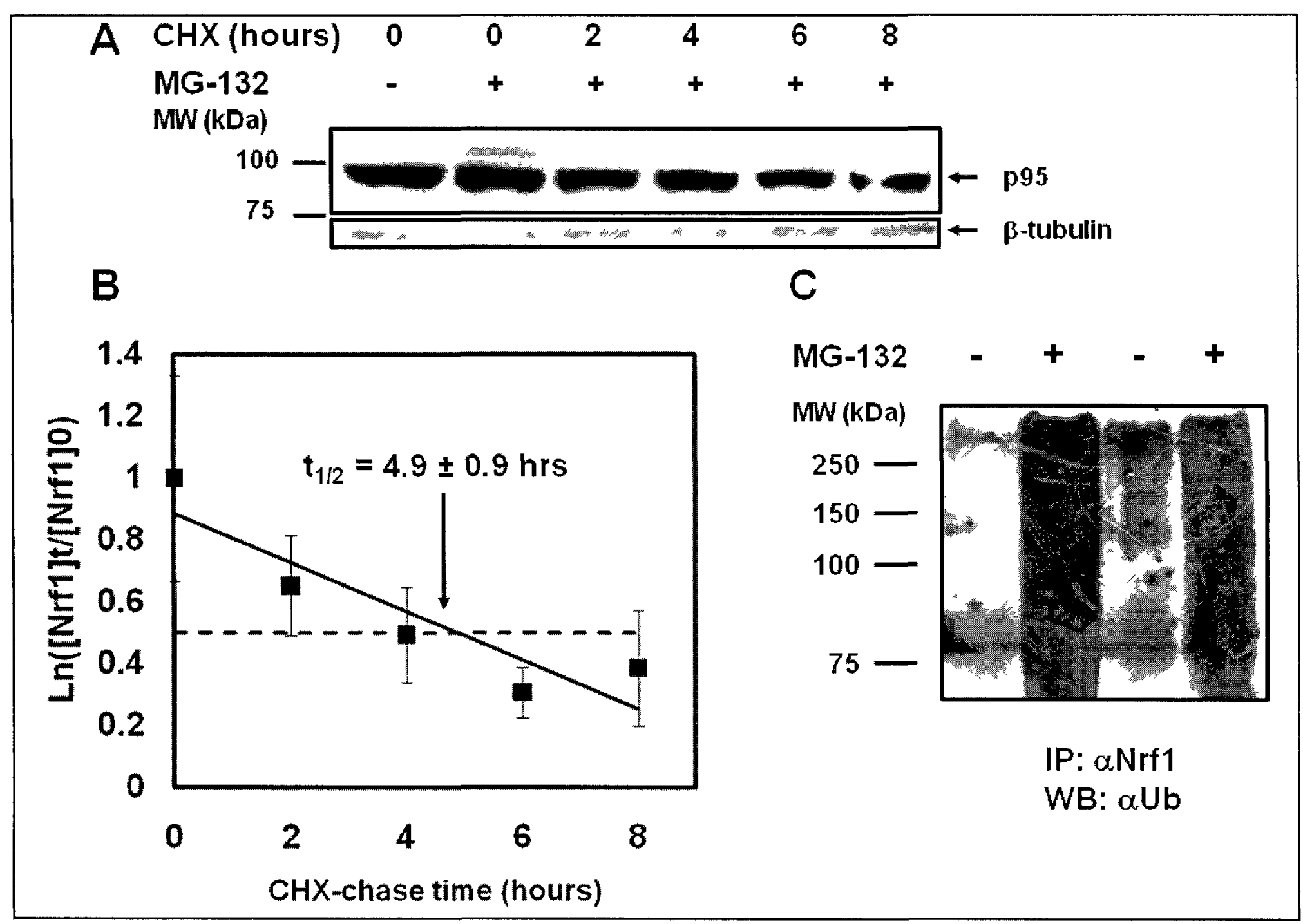


Thus, Nrf1 appears to be precisely controlled by keeping its levels low through its continuous ubiquitination and degradation by the proteasome. Given its relatively long half-life, compared to $\mathrm{Nrf} 2$ and Nrf3, Nrfl may have additional modifications that allow for ubiquitination, similar to the large subunit of RNA polymerase II (RPB1), which requires phosphorylation prior to ubiquitination in order to be degraded by the proteasome (Kuznetsova et al., 2003). Ubiquitination of the target protein would be dependent upon conditions required for the primary post-translational modification to occur. Thus, the turnover of Nrf1 may be higher under conditions that favor other posttranslational modifications.

In addition to ubiquitination, we noticed the disappearance of a high molecular weight form of $\mathrm{Nrfl}$ with an approximate molecular weight of $250 \mathrm{kDa}$ (p250, see Figure 3.4) under stimulated conditions as a result of $\mathrm{CHX}$, oxidative stress (AAPH or tBHQ) and hypoxia treatments. Furthermore, a similar decrease in the p250 content in aged mice tissues was observed using a different antibody, recognizing the C-terminus region of Nrf1 (C-19, Santa Cruz, see Figure 3.4). The identity of this stress-responsive band merits further investigations and could represent post-translational modification of Nrfl (e.g., ubiquitination), Nrf1 homodimer, ER membrane-bound Nrfl or Nrf1, covalently bound to some unknown protein. That such a complex could be observed under reducing and denaturing gel electrophoresis discussion is explained in Section 4.5 of the thesis using previously-published data. 
Figure 3.4. Oxidative Stressors AAPH and tBHQ, Hypoxia, CHX and Aging Destabilize the p250 Form of Nrf1. COS7 cells were treated for six hours with (A) 80 mM AAPH, $100(+)$ or $200(++) \mu \mathrm{M}$ tBHP or hypoxia for twenty-four hours or with (B) $100 \mu \mathrm{g} / \mathrm{mL}$ CHX (Schneider-Poetsch et al., 2010) for the times indicated, after which cells were harvested and total cell lysates were subjected to immunoblotting with antiNrfl or anti- $\beta$-tubulin antibodies. Molecular masses are indicated in $\mathrm{kDa}$. The results of two independent experiments (A) or a representative result of three independent experiments (B) are shown. Nrf1 forms (p65, p95 and p250) are indicated with arrows In (A), $\mathrm{Y}$ and $\mathrm{O}$ refers to mouse liver cytoplasm extract samples from young (6 month) or old (21 month), respectively. Even though the expression of p95 was not seen in the cytoplasmic fraction ("Cytoplasmic"), it was clearly discernable in the nuclear fraction ("Nuclear") and the position of p250 in mouse liver sample was verified using appropriate molecular weight standards. 


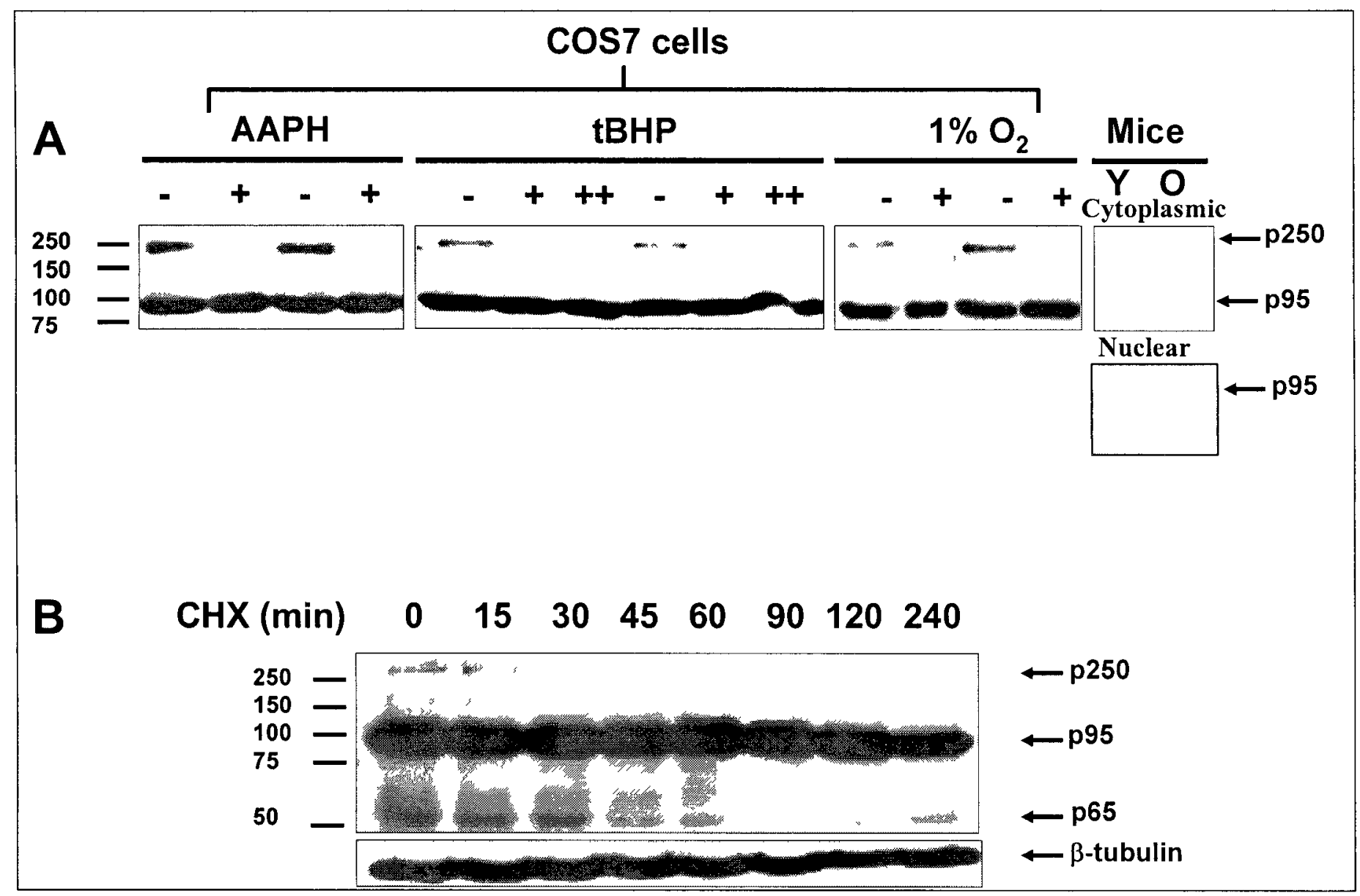




\subsection{Nrf1 Protein Expression under Proteasomal Inhibition and Hypoxia}

With the observation that MG-132 and lactacystin stabilized p120, we wanted to determine if the protein expression of $\mathrm{Nrfl}$ is also affected by hypoxia. To this end, we subjected COS7 and WFF2002 cells to hypoxic conditions $\left(1 \% \mathrm{O}_{2}\right)$. Hypoxia is known to induce the expression of genes involved in iron metabolism, many of which are transcriptionally regulated via the CNC-bZIP factors to which Nrf1 belongs (Chepelev and Willmore, 2011). Similarly, hypoxia activates the expression of metallothioneins (MT) 1 and 2 (Yamasaki et al., 2007); these genes contain the ARE sequence and are known targets of Nrfl, but not Nrf2 (Ohtsuji et al., 2008). Intracellularly, hypoxia treatment has many similarities with proteasomal inhibition; for instance, hypoxiainducible factor alpha subunits (HIFos) are stabilized when the proteasome is inhibited, which leads to HIF $\alpha$-mediated activation of hypoxia-inducible gene expression. In addition, multiple studies have reported an increased rate of ROS production under hypoxia (Klimova and Chandel, 2008) and ROS are known activators of the ARE-Nrfl pathway. Given that hypoxia results in the generation of ROS, we wanted to study the effects of hypoxia on Nrfl function in order to elucidate the mechanisms of Nrf1 regulation in greater detail.

Unlike MG-132 treatment, no stabilization of the p120 form was observed in hypoxic

treatments for both cell lines (Figures 3.5A and B), suggesting that the effect of combining these two treatments is not different from applying each treatment individually. Since the accumulation of the p120 form was also noticeable in WFF2002 
cells (Figure 3.5A), the mechanism of Nrfl control through proteasomal degradation is not likely to be cell-specific. In addition to increased expression of $\mathrm{p} 120$, another form of Nrfl that migrated as a $140 \mathrm{kDa}$ protein was detectable upon immunoblotting (Figure 3.5A). This band was seen in other instances using longer film exposure times and greater amount of protein loaded per gel lane (data not shown). Given the results (described in Figure 3.3C), indicative of Nrfl ubiquitination, it is plausible to suggest that this higher molecular form of Nrf1 represents ubiquitinated form of p120. As ubiquitin is a small protein, comprised of 76 amino acids, and its addition shifts the molecular weight of a protein by approximately $10 \mathrm{kDa}$ (Seyfried et al., 2008), the Nrfl band migrating as an approximately $140-\mathrm{kDa}$ protein could represent mono- or di-ubiquitinated Nrf1.The band density corresponding to p65 Nrfl, a dominant negative inhibitor of ARE-driven gene expression (Wang et al., 2007), was markedly increased after 6 hours of MG-132 treatment in WFF2002 cells (Figure 3.5A) and in both MG-132- and hypoxia-treated COS7 cells (Figure 3.5B). Very little is currently known about p65 and its role in AREmediated gene expression, apart from its negative effect on the ARE pathway and cell type-specific accumulation of p65 might provide some hints towards its function and regulation. We observed no changes at the mRNA levels for all four conditions tested (data not shown), supporting the involvement of post-translational modifications in Nrfl regulation by the proteasome and hypoxia.

Interestingly, in both cell lines, the intensity of the band migrating as a $23 \mathrm{kDa}$ protein ("p23" Nrf1) was diminished during proteasomal inhibition (see Figures 3.5A and B). Since our anti-Nrfl antibody recognizes the N-terminal fragment of Nrfl (amino 
acids 191-475) and p23 is always highly expressed, this suggests that this is a product of $\mathrm{N}$-terminal cleavage, in addition to the ubiquitin-dependent proteasomal degradation discussed above, p23 likely represents either protease- and/or a stable, proteasomegenerated N-terminal fragment of Nrf1. A band of approximately the same molecular weight as p23 is also observed using another antibody, H-285 (Santa-Cruz, http://www.scbt.com/datasheet-13031-nrf1-h-285-antibody.html), which confirms that p23 is a fragment of $\mathrm{Nrfl}$ and not some non-specific protein, cross-reacted with the antibody used. That $\mathrm{Nrf1}$ is processed at its $\mathrm{N}$-terminus is in line with the previous studies, suggesting that p120 Nrf1 must be processed into smaller forms in order to remove the inhibitory NTD and allow the protein to act as a transcription factor (see (Schultz et al., 2010) and references therein). The N-terminal cleavage of Nrfl might be an important way of regulating this factor as that would allow it to escape the ER and to translocate to the nucleus, where it could activate the expression of the ARE-controlled genes. The conclusion that $\mathrm{p} 23$ is indeed a Nrfl fragment was based on the following: i) the N-terminal location of the Nrfl region, recognized by the antibody employed; ii) the observation that $\mathrm{p} 23$ signal was diminished following proteasomal inhibition in both COS7 and WFF2002 cells (Figure 3.5), with concomitant increase in the p120 Nrfl; and iii) the fact that the overexpression of the Nrfl, FLAG-tagged at its N-terminus was able to transactivate the ARE-luciferase reporter, but could not be seen following FLAG immunoblotting (data not shown), unlike the Nrfl, FLAG-tagged at its C-terminus that can be visualized by using anti-FLAG antibody as will be shown. 
Figure 3.5. Comparison of the Nrf1 Expression under Hypoxia and Proteasomal Inhibition. WFF2002 (A) and $\operatorname{COS} 7$ (B) cells were treated with normoxia $\left(21 \% \mathrm{O}_{2}\right)$, hypoxia $\left(1 \% \mathrm{O}_{2}\right), 10 \mu \mathrm{M}$ MG-132 or hypoxia and MG-132 combined for six hours and subjected to Western blotting. The band density was normalized with respect to $\beta$-tubulin or Ponceau $\mathrm{S}$ red and is presented as means \pm S.E.M. of at least three independent experiments for WFF2002 (C) and COS7 (D) cells. Asterisks (*) indicate significant difference $(p<0.05)$ compared to controls using Student's t-test. 


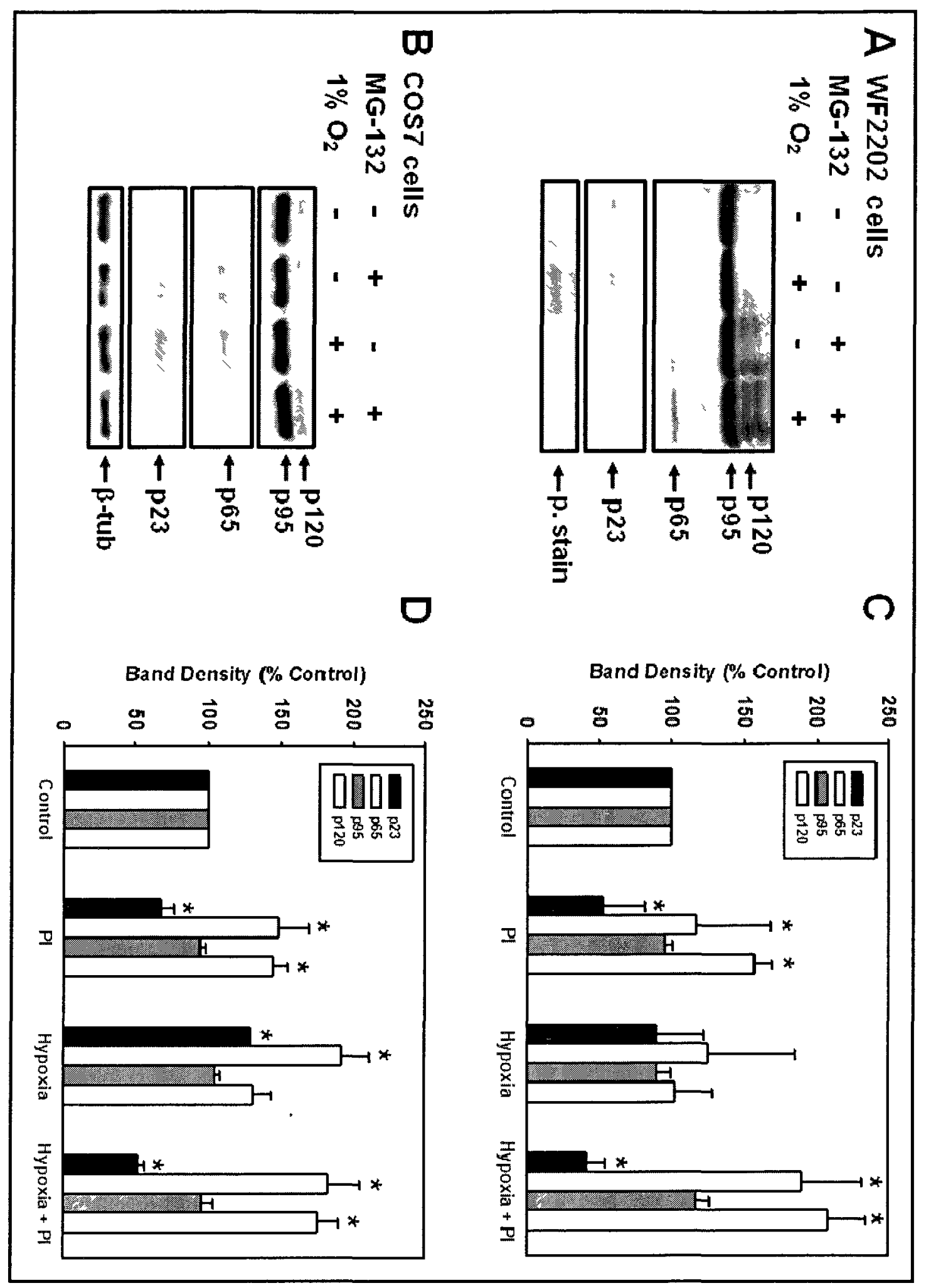


Other ER-bound transcriptional factors are similarly regulated through intermembrane proteolysis and include the sterol-regulatory-element-binding proteins SREBP1 and SREBP2, as well as activating transcription factor 6 (ATF6), and this regulation has been well-characterized (see Wang and Chan, 2006), and references therein). Previous studies (Zhang et al., 2007; Zhang and Hayes, 2010) failed to map any proteolytic cleavage site within the first 170 amino acids at the $\mathrm{N}$-terminus; however, the studies were performed at normal homeostatic conditions and the possibility of Nrf1 regulation by intramembrane proteolysis under stimulated conditions cannot be ruled out (Zhang and Hayes, 2010). In contrast, a more recent study (Steffen et al., 2010) provided some, albeit indirect, support for the intramembrane proteolysis of Nrfl prior to its translocation to the nucleus from the ER.

\subsection{MG-132 and Hypoxia Enhances Protein Binding to the ARE}

Once we showed that proteasomal inhibition stabilizes Nrfl, we investigated whether MG-132 and/or hypoxia have any effects on Nrfl DNA-binding to the ARE. Using electrophoretic mobility shift assays (EMSAs), we observed the appearance of MG-132and hypoxia-inducible bands (Figure 3.6A). These results are consistent with the previously reported study of Waleh and co-workers (1998), showing hypoxia-inducible DNA-binding to AREs in human HepG2 and mouse Hepa cells. 
Figure 3.6. Hypoxia and Proteasomal Inhibition Increase Protein Binding to the ARE. Biotin-labelled ARE probe from the $\mathrm{gclm}$ promoter was applied to $20 \mu \mathrm{g}$ of total $\operatorname{COS} 7$ cell lysate protein in an EMSA format as described in the Methods Section, Chapter 2. (A) Depicts the appearance of the hypoxia- and MG-132-inducible band. C, $\mathrm{PI}, \mathrm{H}$ and $\mathrm{PI}+\mathrm{H}$ designate control (DMSO), proteasomal inhibition (MG-132), hypoxia $\left(1 \% \mathrm{O}_{2}\right.$ and DMSO) and the combination of hypoxia and MG-132 treatments, respectively. (B) The identity of the ARE-bound complex was probed using antibodies against the proteins indicated in the immunodepletion format. $F$, free probe; $I$, inducible band; S, supershift. Maf refers to an antibody, recognizing all three forms of small Maf proteins (MafF, G and K). 


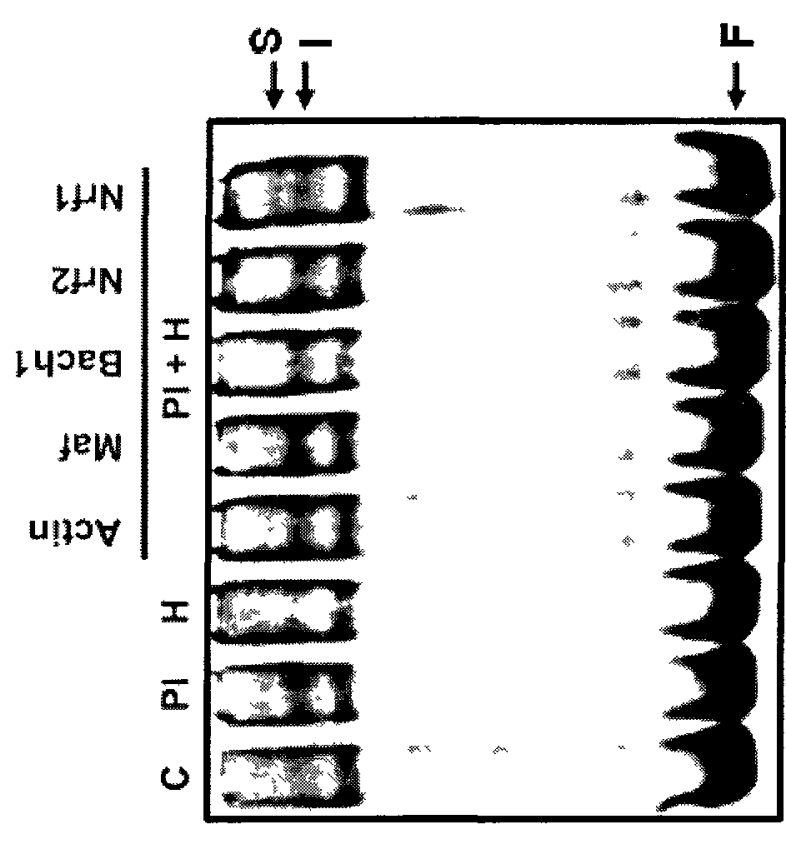

$m$

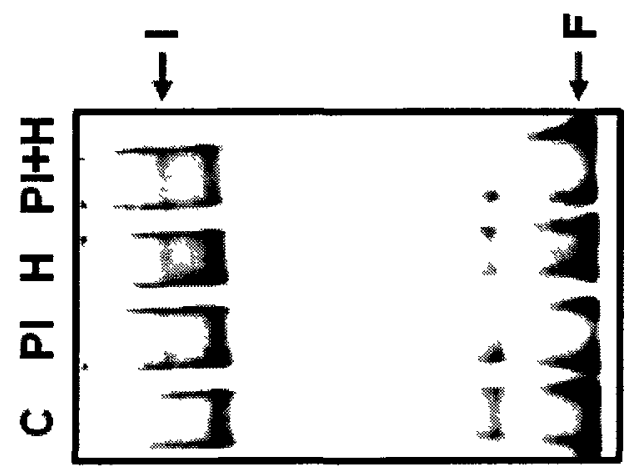

4 
To identitfy the proteins responsible for the hypoxia- and MG-132-inducible AREbinding, we used an immunodepletion approach (Dickinson et al., 2003). In this method, an antibody raised against a DNA-binding protein diminishes the intensity of an EMSA band if the DNA-binding protein is, indeed, bound to the target DNA sequence under investigation (Dickinson et al., 2003). Apart from Nrfl, the obvious candidate factors that could be involved in the MG-132- and hypoxia-inducible ARE complex formation could be $\mathrm{Nrf} 2$ as it is stabilized by proteasomal inhibition and Bach1, whose activation by hypoxia has been previously described (Kitamuro et al., 2003). As can be seen from Figure 3.6B, anti-Nrfl antibody resulted in the appearance of an "upshift" or "supershift", not clearly discernable when other antibodies were used. This suggests potential involvement of Nrf1 in the hypoxia- and MG-132-inducible binding to an ARE probe. However, the MG-132- and hypoxia-inducible band was still robust following the anti-Nrfl antibody addition, suggesting that other proteins may be involved in the increased ARE binding observed here. These other proteins could be some unknown ARE-binding proteins, small Mafs or other CNC-bZIP factors, known to bind to the ARE, whose binding was not seen in this experiment probably because an antibody, bound to a DNA-binding protein can block the protein-DNA interaction. Since enhanced ARE binding could result in either activation or repression of the ARE target genes, depending on the nature of the binding factor involved (activator or repressor), we used a luciferase reporter assay, in combination with $\mathrm{Nrfl}$ overexpression, to gain a better understanding of the Nrfl transactivation function in response to hypoxia and proteasomal inhibition treatments. 


\subsection{Hypoxia Activates and Proteasomal Inhibitors Inhibit Exogenous Nrf1}

\section{Activity}

We tested if proteasomal inhibition and hypoxia have any effect on the transactivation activity of Nrfl. To this end, we used a luciferase reporter vector under the control of three AREs from chicken $\beta$-globin enhancer (3xARE-luciferase (Igarashi et al., 1994)). Transient co-transfection of 3xARE-luciferase with Nrfl-FLAG in COS7 cells showed that 24-hour hypoxia treatments greatly increased the activity of Nrfl-FLAG (Figure 3.7A). Furthermore, hypoxic activation of Nrf1 is probably not cell type-specific as the same results were seen in HEK293A cells (Figure 3.7A). Neither does Nrf1 hypoxic inducibility seem to be an intrinsic property of this particular CNC-bZIP family member as exogenous $\mathrm{Nrf} 2$, overexpressed in $\operatorname{COS} 7$ cells, also responded to $1 \% \mathrm{O}_{2}$ in a manner, similar to Nrfl (Figure 3.7A). In addition to low oxygen, Nrfl-FLAG activation is achievable by hypoxia mimetics cobalt chloride and dimethyloxalylglycine (DMOG, Figure 3.7C). In contrast, MG-132 markedly decreased the activity of Nrf1-FLAG while hypoxia had no effect on the Nrfl activity during six-hour treatments (Figure 3.7B). Again, the action of MG-132 on exogenous Nrfl activity can be understood in light of the current hypothesis that the processing of $\mathrm{Nrfl}$ by the $26 \mathrm{~S}$ proteasome could remove its inhibitory NTD (see Figure 1.4 for Nrfl domains), targeting Nrfl to the ER. Blocking Nrf1 processing into p23 and other active forms of Nrf1 (such as p95) by proteasome inhibition would interfere with Nrfl function, which is in accord with the observed repression of the Nrfl activity by MG-132 treatment (Figure 3.7B). Therefore, proteasomal processing seems to be a prerequisite for Nrfl activation and function. 
Figure 3.7. Hypoxia and Hypoxic Mimetics Activate Nrf1, while MG-132 Inhibits its Transactivation Activity. COS7 and HEK293A cells were co-transfected with 3XAREluc, $\beta$-galactosidase and Nrf1-FLAG or Nrf2-myc plasmids, and treated with hypoxia, 10 $\mu \mathrm{M}$ MG-132, $2.5 \mathrm{mM}$ DMOG or $200 \mu \mathrm{M} \mathrm{CoCl} 2$ for twenty-four ((A) and (C)) or six (B) hours as indicated and the luciferase activity in the lysates was measured. The pCR3.1 plasmid, lacking $\mathrm{Nrfl}$ and Nrf2, was used as a negative control and $\beta$-galactosidase activity was used to determine transfection efficiency. The means of at least three independent experiments \pm S.E.M., normalized to the $\beta$-galactosidase activity and pCR3.1, are presented. Asterisks $(*, * *)$ indicate significant difference $(\mathrm{p}<0.05$ or $\mathrm{p}<$ 0.001 , respectively) compared to controls. The lysates of hypoxia-treated $\left(1 \% \mathrm{O}_{2}, 24\right.$ hours) COS7 (D) and HEK293A (E) and (F) cells were also subjected to immunoblotting using anti-FLAG antibody. In (D) and (E), asterisks (*) denote the non-specific, crossreacting bands. Representative blots of three independent experiments are shown. 


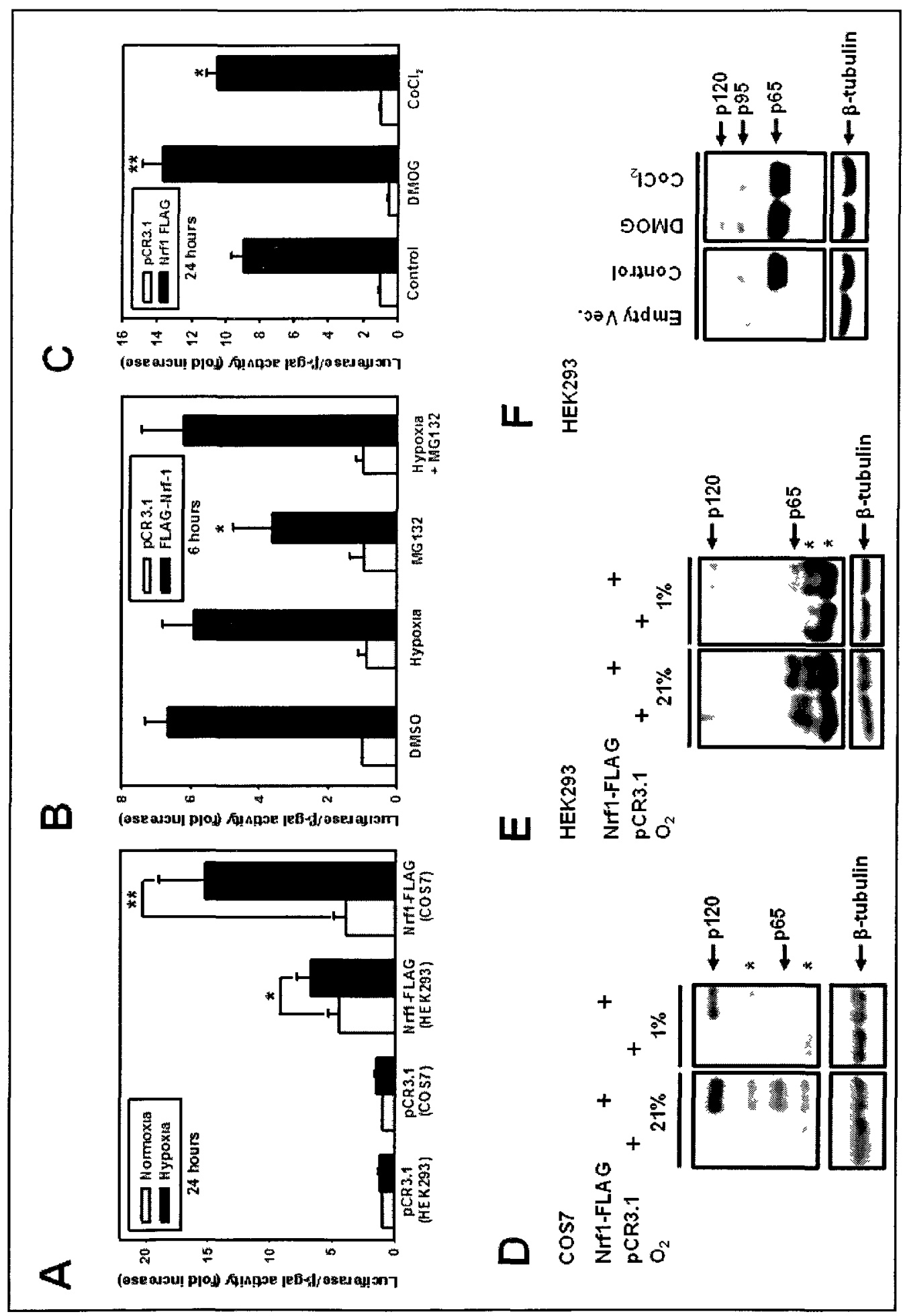




\subsection{Potential Effect of p65 Nrf1 on the Hypoxic Inducibility of Nrf1}

The p65 form of Nrfl is thought to be produced by either internal proteolysis (Zhang et al., 2007) or as a result of translation initiation from an alternative Met codon (Chan et al., 1993). The p65 $\mathrm{Nrfl}$ form is thought to act as a dominant negative inhibitor of the ARE-driven gene expression (Wang et al., 2007) as it contains a DNA-binding domain, but lacks a transactivation domain, such that p65 Nrfl competes with Nrf2 for the ARE binding site. As seen in Figures 3.7D and E, the expression of the p65 form of Nrfl markedly decreased during hypoxia treatment in both COS7 and HEK293A cell lines, while no other common change was noticeable during hypoxia. Our data presented here is in agreement with the model in which the ARE-mediated gene expression is activated as a result of down-regulation of the inhibitory p65 form of Nrfl. The two internal Met residues that are thought to give rise to p65 are M321 and M326 (Chan et al., 1993) which contain consensus Kozak sequences, not present in the M1 codon. Mutating M321 and M326 to M321L and M326L, respectively, resulted in significant loss of basal Nrf1FLAG activity and hypoxia inducibility; however, unexpectedly, mutants were not expressed at detectable levels (data not shown). This may be due to aggregation of mutant forms of the protein. Alternatively, this apparent lack of expression and any transactivation activity by the mutants could be explained by their increased instability. No significant effect of the transfection with the mutant-bearing plasmid on the cell viability was seen using the MTT assay or $\beta$-galactosidase activity (data not shown). 
Figure 3.8. Phosphorylation Activates Nrf1 and Antioxidant Treatment Only Partially Abolishes the Hypoxic Inducibility of Nrf1. The luciferase assays were performed on the COS7 cell lysates, co-transfected with 3XARE-luciferase, and Nrf1FLAG plasmids treated with (A) okadaic acid (OA) and staurosporine (SS), to inhibit protein dephosphorylation and phosphorylation, respectively, or with a combination of an antioxidant NAC and hypoxia (B) for twenty-four hours. DMSO or ethyl acetate (EA) was used as vehicle controls (V) in and the concentration of each compound used is indicated in (A). The means of at least three independent experiments \pm S.E.M. are shown. Asterisks $\left(^{*}\right)$ indicate significant differences $(p<0.05)$ between indicated pairs or controls as calculated using paired Student's t-test. 


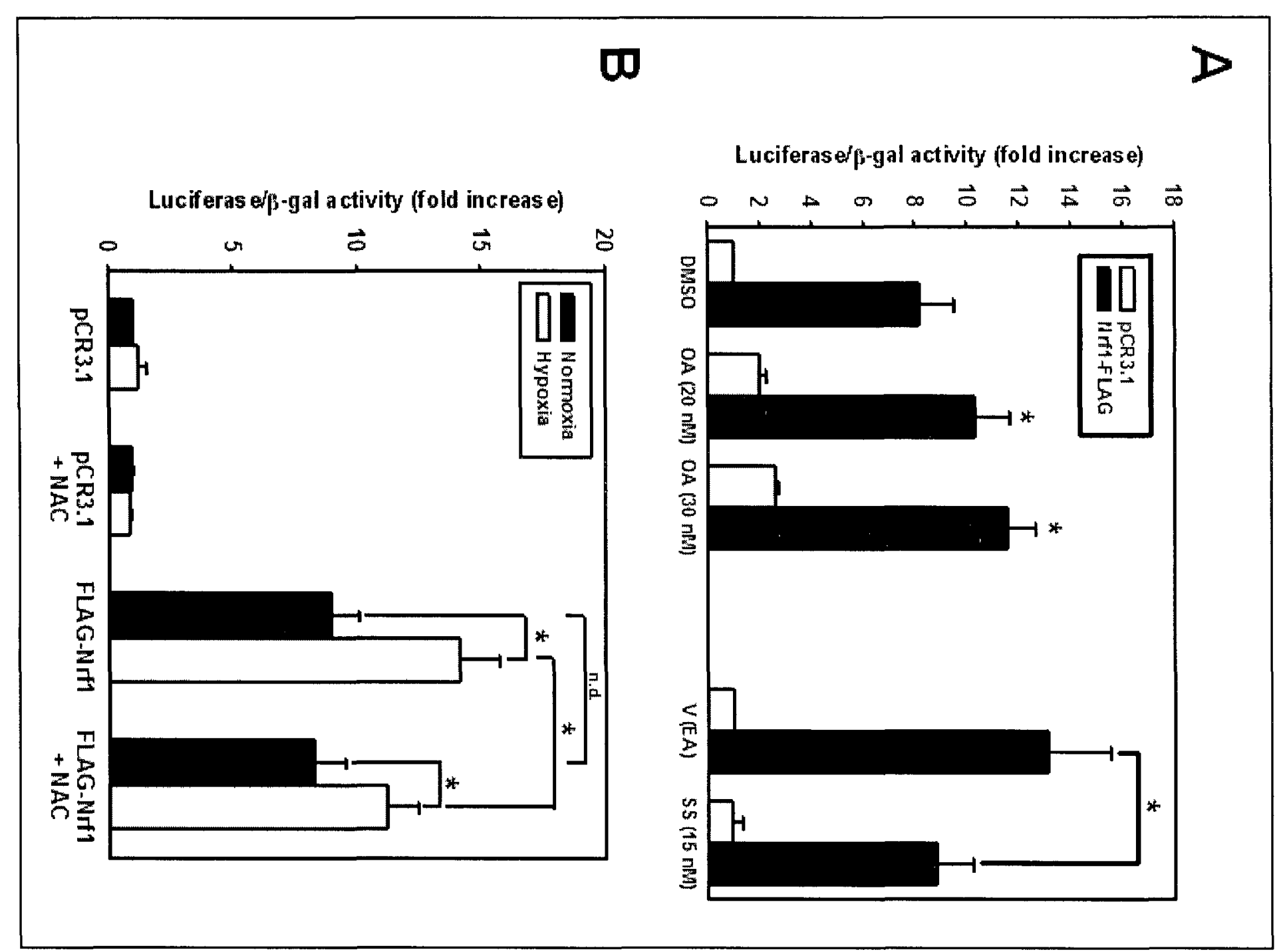




\subsection{Nrf1 is Activated by Phosphorylation}

Our quick computational analyses predicted a high probability of Nrfl phosphorylation by cdc2 and protein kinase C (PKC) members, especially at the Ser-rich region (NST), facing the ER lumen. Zhang and others (2009) provided some evidence that phosphorylation of Nrfl at its NTD (which contains two potential Tyr phosphorylation motifs, amino acids $62-70$ in human Nrf1), may weaken the Nrf1 association with the ER, stimulating the Nrfl trafficking to the nucleus. Given that phosphorylation by atypical PKC is known to activate Nrf2 (Numazawa et al., 2003), we thought that Nrf1 could also be a subject to this modification. To test that, we performed luciferase assays on the COS7 cells co-transfected with Nrfl-FLAG and 3xARE-luciferase and subjected to protein phosphatase inhibitor okadaic acid (OA) (Nguyen et al., 2003; Zhang and Forman, 2008) or pan-PKC inhibitor staurosporine (SS) (Zhang et al., 2008). Figure 3.8A illustrates that OA activated Nrf1, while SS repressed Nrf1 activity, suggesting that Nrf1 phosphorylation plays a role in Nrf1 transactivation. Notably, the OA treatment resulted in about 2-fold increase of the empty vector (pCR3.1)-mediated ARE-luciferase activation, changing the ARE-driven luciferase activity from 1.0- to 2.0-fold with respect to the control vector. This suggests that there is about 1-fold increase in the "noise" signal due to other, transcriptional factors, such as Nrf2, being activated through phosphorylation. However, treatment with OA changed the ARE-luciferase reporter activity due to Nrfl-FLAG from $8.15 \pm 0.7$ - to $10.3 \pm 0.6$ - and $11.6 \pm 0.6$-fold for 0,10 and 20-nM treatments with OA, respectively, compared to empty vector-transfected controls (pCR3.1). Therefore, the net increase in Nrfl-FLAG activity was 2.15 -fold 
compared to 1 -fold increase due to "noise". These results, as well as a similar study on the effect of OA on $\mathrm{Nrf} 2$ (where an approximate 0.5 -fold increase in the $\mathrm{Nrf} 2$ activity due to OA treatment above background was interpreted as a stimulatory effect of OA on Nrf2 activity (see Figure 6 in Nguyen et al., 2003), suggests that Nrfl positively responds to phosphorylation stimulus. Decreased transactivation activity of $\mathrm{Nrfl}$, as a result of SS treatment, strengthens the claim that the phosphorylation status of Nrfl is important for Nrfl activity. It is possible that some upstream factors in the signaling cascade that control Nrfl function responds to phosphorylation/dephosphorylation rather than Nrfl itself and further studies are required to confirm the target of phosphorylation in the Nrfl pathway.

\subsection{The Potential Involvement of ROS in Hypoxic Inducibility of Nrf1}

Low oxygen conditions are known to increase ROS production from mitochondrial electron-transport chain (reviewed in Klimova and Chandel, 2008). To test if increased ROS levels could be responsible for Nrf1 activation, we treated Nrf1-FLAG- and 3xARE-luciferase-transfected COS7 cells with hypoxia in combination with the antioxidant $\mathrm{N}$-acetylcysteine (NAC). As seen from Figure 3.8B, induction of luciferase activity by hypoxia was only partially abolished with NAC. The data suggest that ROS may, at least in part, contribute to hypoxic activation of exogenous Nrfl. The existence of other mechanisms responsible for $\mathrm{Nrfl}$ activation, however, cannot be ruled out.

\subsection{The Two Proposed Levels of the Proteasomal Control of Nrf1}


The levels of key inducible transcription factors are normally kept low under homeostatic conditions, through their ubiquitination and continuous degradation by the proteasome, to avoid aberrant gene expression. This is the case for transcription factors such as HIF $\alpha$ and the CNC-bZIP factors Nrf2 and Nrf3. As expected, we found that Nrfl was also negatively regulated by the proteasome, being subject to ubiquitination and proteasomal degradation in agreement with Zhang and co-workers, who suggested that Nrfl abundance may be controlled by the proteasome (Zhang et al., 2009). However, given the rather high half-life of $\mathrm{Nrfl}$, compared to $\mathrm{Nrf} 2$ and $\mathrm{Nrf3}$, the existence of other regulatory mechanisms for Nrf1 is very likely. One might wonder how the proteasome, which is typically localized to the cytoplasm and nucleus, can come into contact with membranebound Nrfl. Perhaps, Nrfl could be partially processed by the proteasome once p95 has been glycosylated to become $\mathrm{p} 120$ and prior to its integration in the ER membrane as has been suggested to be the case for Nrf3 (Chevillard and Blank, 2011). We observed high expression of the p23 fragment of Nrfl under untreated conditions, which diminished following MG-132 treatment and provides further evidence that the p23 is derived from Nrfl and does not represent nonspecific binding of the antibody to some other protein. Since p23 would be expected to contain about 210 amino acids, and the recognition region of our antibody is between amino acids $191-475$, we believe that p23 represents an N-terminal fragment of the Nrfl, cleaved twice in the amino acids 191-475 region, with the distance between the two cleavage sites being approximately 210 amino acids. Further support of this hypothesis has been shown in previous experiments, where Site-1 or Site-2 protease cleavage sites within the first 170 amino acids of Nrfl could not be 
identified (Zhang et al., 2007; Zhang and Hayes, 2010). Unlike the multicatalytic proteasome complexes, the membrane-bound Ser peptidases Site-1 and Site-2 are metalloproteases, which recognize the general motifs $\mathrm{R}-(\mathrm{R} / \mathrm{K} / \mathrm{H})-\mathrm{L}-(\mathrm{A} / \mathrm{L} / \mathrm{S} / \mathrm{T} / \mathrm{F}$, Site-1) and GPxxN/S/G or NxxPxxxxDG (Site-2, where $x$ stands for any amino acid) on their protein substrates, (Rojek et al., 2008; Kinch et al., 2006) and participate in two-step cleavage of their substrates, such as SREBP1 (Zhang et al., 2006). In addition to Site-1 and Site- 2 proteases, the proteasome can also act as a site-specific protease that induces only partial as opposed to global protein cleavage of proteins, leading to their complete degradation, which is a more common function of the proteasome. An example of sitespecific, partial proteolysis carried out by the proteasome is he proteasomal cleavage of $\mathrm{NF}-\kappa \mathrm{B}$ as described in greater detail further in this section. Our results support the hypothesis of Zhang and co-workers (Zhang et al., 2007) that Nrfl is proteolytically cleaved at regions other than the NTD. Cleavage by Site-1 and Site- 2 intramembrane proteases is a well-known mechanism, responsible for the release of ER-bound transcriptional factors, but the possibility that Nrf1 is cleaved at the AD1, NST and AD2 domains merits further investigation. This possibility is also supported by Steffen and coworkers (2010), who noticed that the molecular weight of the unglycosylated Nrfl is still higher than that of the nuclear form of $\mathrm{Nrfl}$ and concluded that intramembrane proteolysis is a feasible mechanism for Nrfl release to the nucleus from the ER.

The second or "positive" level of Nrfl regulation by the proteasome, in addition to complete ubiquitin-mediated proteasomal degradation, appears to be partial proteolytic processing by the proteasome. In accord with the current hypothesis of the Nrfl 
regulation by removal of the NTD domain from full-length Nrfl (Schultz et al., 2010), proteolytic cleavage of Nrf1 allows it to bypass insertion into the ER and travel to the nucleus. This hypothesis was supported by the fact that proteasomal inhibition with MG132 not only stabilized p120 Nrfl and decreased p23 levels, but also repressed Nrf1 activity. A very similar mode of activation, through the processing of p105 to the DNAbinding, p50 form of NF- $\mathrm{kB}$ by the $26 \mathrm{~S}$ proteasome, has been described (Magnani et al., 2001; Tanaka et al., 2000). A recent study has demonstrated the involvement of Nrf1 in the "bounce-back" response, an elevated proteasomal subunit synthesis which is observed upon proteasome inhibition (Radhakrishnan et al., 2010). According to the study, Nrfl directly activates the proteasome recovery pathway upon proteasome inhibition. For that to happen, Nrfl itself must be activated by $1 \mu \mathrm{M}$ MG-132 treatment, which was shown by the investigators (Radhakrishnan et al., 2010). This is in contrast to our results, as well as the results of others (see Figure 2 of Zhang et al., 2009), demonstrating that MG-132 and another proteasome inhibitor, ALLN, applied at 10 or $13 \mu \mathrm{M}$ concentrations respectively, represses exogenous $\mathrm{Nrfl}$ activity. While both 1 and 10-13 $\mu \mathrm{M}$ concentrations of the proteasome inhibitors were able to stabilize p120 Nrfl, abolishing the proteasomal degradation of Nrfl completely, it is conceivable that higher concentrations of proteasomal inhibitors are required for the inhibition of the Nrf1 partial processing/activation by the proteasome (which is known to possess several catalytic activities). Thus, it can be envisioned that the stimulatory effect of a proteasomal inhibitor on Nrfl activity, resulting from its blockade of the proteasomal degradation of $\mathrm{Nrfl}$, can be outweighed by the repression of the proteasome-mediated activation of Nrfl through partial proteolytic processing at higher $(\geq 10 \mu \mathrm{M})$ proteasome inhibitor 
concentrations. Another possible explanation for this discrepancy is the fact that reporter plasmids with AREs from different genes (utilizing different promoters) were used in these different studies and while one set of ARE-controlled genes can be turned on by a given conditions, other ARE-driven genes can be turned off due to the complex interplay between co-activator and co-repressor proteins (Zhou and Fahl, 2001). Testing a wider range of MG-132 concentrations could have provided useful insights with respect to Nrfl and mechanisms of negative and positive regulation by the proteasome

\subsection{Potential Significance of the Stress-inducible Nrf1 Forms}

Our findings have emphasized the role of multiple forms of Nrf1. Indeed, Nrfl is known to exist in several forms, including p120 (glycosylated Nrfl), p95 (non- or unglycosylated Nrf1), inhibitory p65 form as well as 46 and $30 \mathrm{kDa}$ forms (Zhang et al., 2009). In addition to these, we report the existence of the $\mathrm{p} 23 \mathrm{Nrfl}$ form, that seems to be a fragment of proteasomal processing of the full-length Nrf1 and another, high molecular weight p250 Nrfl form, which is destabilized by oxidative stressors, inhibition of protein synthesis and hypoxia. Since the p250 form responds to multiple stimuli, its characterization will be useful in further attempts to uncover the mechanisms responsible for Nrf1 activation. It is plausible that the p 250 form of Nrfl represents a covalently linked protein-protein or protein-membrane interaction with Nrfl, or a Nrfl dimer. That it is possible to observe dimer formation on the SDS-PAGE will be discussed in greater 
detail in Section 4.5. Another possibility is that $\mathrm{p} 250$ is a currently uncharacterized, posttranslationally modified, form of Nrf1 such as polyubiquitinated Nrfl.

\subsection{Hypoxic Activation of Nrf1 is Accompanied by p65 Nrf1 Down-regulation}

This is the first study to report Nrfl activation by hypoxia. As it has been speculated that the location of Nrf1 in the ER membrane is suitable for the maintenance of the homeostatic redox status of the ER (Zhang et al., 2009), the finding that Nrfl is a hypoxia-inducible factor is not surprising, given that hypoxia is known to cause the ER stress due to the accumulation of the unfolded proteins in the ER lumen (Mera et al., 2010). Among the genes controlled exclusively by Nrf1 (and not Nrf2) are the metallothioneins (MT 1 and 2) (Ohtsuji et al., 2008). It was found that MT expression is hypoxia-inducible in PCa cells (Yamasaki et al., 2007) and, according to our study, we think that Nrfl hypoxic inducibility could be responsible for MT upregulation in response to hypoxia. Nrfl processing must be highly regulated as its full-length p95 form can act as an activator of the ARE-driven gene expression while its shorter form, p65, acts as an apparent inhibitor of the ARE pathway. Using transient overexpression of Nrfl-FLAG, we noticed that the levels of the p65 Nrfl were significantly lowered by hypoxia, suggesting that the cells possess the ability to control Nrfl activity by removal of the inhibitory p65 form. The involvement of other mechanisms such as phosphorylation in the hypoxic inducibility of Nrfl can further augment activation of this CNC-bZIP factor. 


\subsection{The Proposed Model for the Nrf1 Regulation by Hypoxia and the Proteasome}

This study has opened up new avenues of research regarding the regulation of Nrfl. What is currently unknown is the identity of the kinase(s) and the Nrfl residue(s) involved in Nrfl phosphorylation. Zhang and co-workers (2009) speculated that Nrf1 phosphorylation can take place at Tyr65 and Tyr77, which could weaken Nrfl affinity for the ER membrane and stimulate its nuclear import. As well, the proteolytic processing of Nrf1 requires further investigation to identify the protease responsible for that as well as the site of the cleavage. Furthermore, it is currently unknown how phosphorylation and processing of Nrf are controlled and if the two processes are interdependent. Given that the p250 form of Nrfl responds to various stimuli, it would be extremely informative to determine whether this form represents a hyperglycosylated or otherwise modified form of $\mathrm{Nrfl}$, why this form is destabilized under stimulated conditions and what is the significance of the destabilization.

In conclusion, our current working model of Nrf1 regulation is summarized in Figure 3.9. According to this model, Nrfl is a subject to several regulatory events, including: i) negative regulation by the proteasome through ubiquitin-mediated degradation; ii) positive regulation by the proteasome through partial proteolytic processing to generate the $\mathrm{p} 23$ fragment; iii) phosphorylation and iv) derepression of its transactivation activity by the removal of the inhibitory p $65 \mathrm{Nrfl}$ form. These putative mechanisms can be investigated 1) by site-directed mutagenesis to reveal the location of 
the cleavage, 2) by affinity purification followed by mass spectrometry to reveal the sites of ubiquitin attachment and phosphorylation, as well as the sites of proteolytic cleavage and 3) by site-directed mutagenesis in an attempt to prevent the hypothesized translation of the $\mathrm{Nrfl}$ gene from an internal Met codon. 
Figure 3.9. The Proposed Working Model for the Nrf1 Regulation by the Proteasome and Oxygen. Nrf1 is controlled at the level of protein degradation by ubiquitin $(\mathrm{Ub})$-mediated proteasomal degradation such that the half-life of Nrfl is approximately five hours. In addition, Nrfl is also a subject of proteasome-mediated proteolysis during which its N-terminus, containing the inhibitory NTD, is cleaved to convert Nrfl from inactive, ER-bound p120 form to the active, nuclear p95 form, releasing the N-terminal Nrfl fragment, p23. Nrfl can be also activated through its phosphorylation or the phosphorylation of some other protein, which may affect $\mathrm{Nrfl}$ activity indirectly. Through a currently unknown mechanism, hypoxia acts on p65 Nrf1 to diminish its expression. This relieves the Nrfl p65-mediated repression (by the removal of p65) on the p95 transactivation activity which, in combination with p95 activation by phosphorylation, may greatly affect the expression of Nrfl-ARE target genes. 


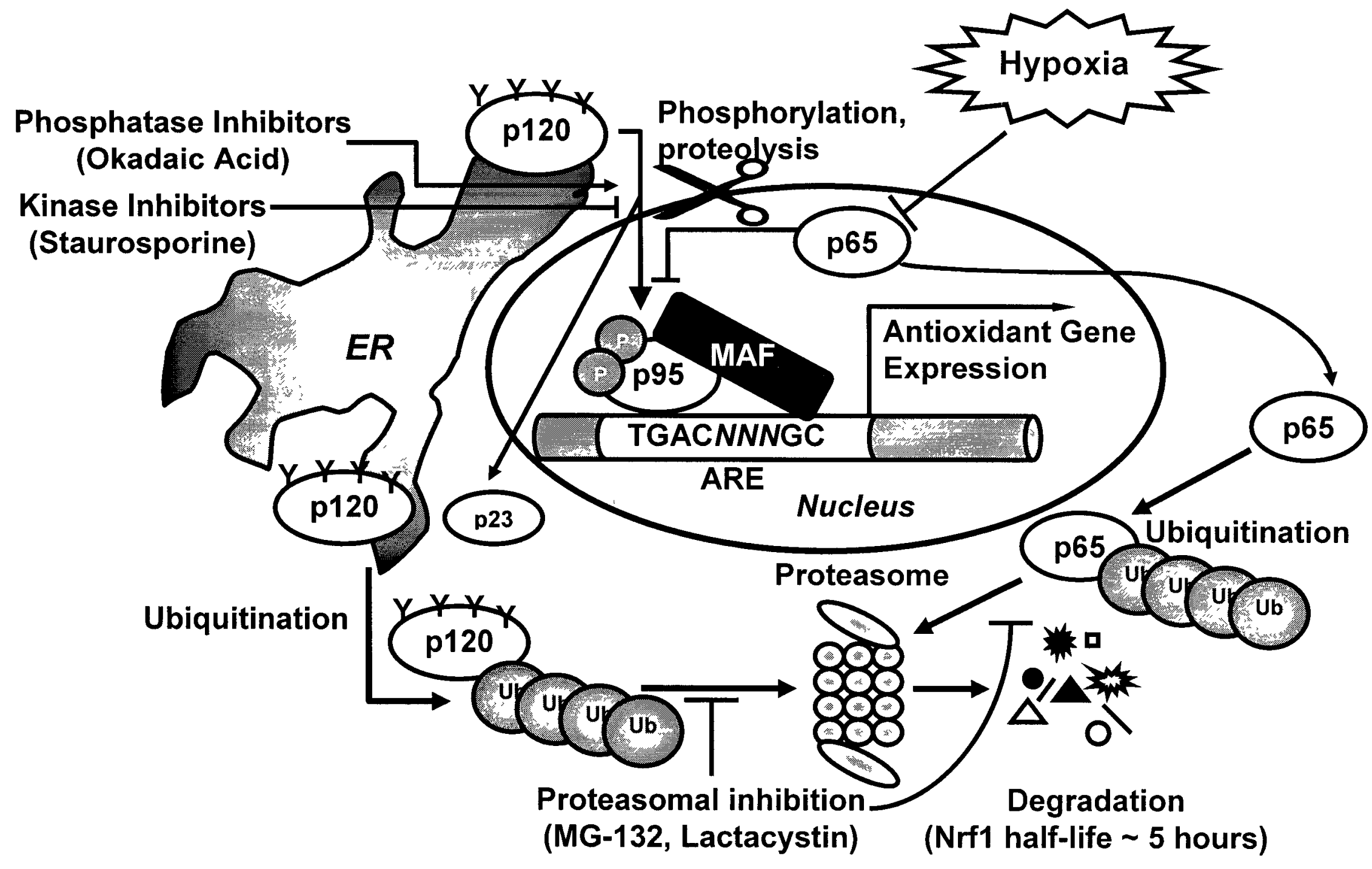


4 Chapter: The Effect of BPA on the Nrf1/2-ARE Pathway 


\subsection{BPA and its Relation to the ROS Generation and Antioxidant Enzymes}

Bisphenol A (BPA) is used in the production of polycarbonate plastics and epoxy resins for baby bottles, liners of canned food and many other consumer products. Previously, BPA has been shown to reduce the activity of several antioxidant enzymes, which may contribute to oxidative stress. However, the underlying mechanism of the BPA-mediated effect upon antioxidant enzyme activity is unknown. BPA has been reported to reduce the activities of catalase and glutathione peroxidase in cultured cells (Oh and Lim, 2008), mice (Kabuto et al., 2003) and rats (Chitra et al., 2003), resulting in oxidative stress conditions. Other pro-oxidant effects of BPA include DNA breakage in MCF-7 cells (Iso et al., 2006). Given the reported relationship between BPA, ROS generation and antioxidant enzymes, we wanted to investigate the effects of BPA on the ARE/EpRE pathway in human embryonic kidney (HEK 293) cells.

Despite previous reports on the pro-oxidant properties of BPA, we predicted that BPA would have antioxidant properties, given that it is a polyphenolic compound and polyphenols are potent antioxidants. Thus, a theoretical prediction of the antioxidant properties of BPA was attempted. A good antioxidant is expected to sacrifice an electron, usually from a weak R-H bond to reduce the attacking species, after which an antioxidant would either be regenerated by compounds such as ascorbate, or undergo a disproportionation reaction to form unreactive species, terminating the proliferation of oxidative damage. The strength of the sacrificial $\mathrm{R}-\mathrm{H}$ bond has to be sufficiently low to allow for the quenching of peroxyl radicals (ROO•) through the regeneration of the 
ROO-H bond, the BDE of which is approximately $88 \mathrm{kcal} / \mathrm{mol}$. For the regeneration reaction to be favorable, the sacrificial $\mathrm{R}-\mathrm{H}$ BDE has to be below that of the $\mathrm{ROO}-\mathrm{H}$ bond. It is also desirable for the antioxidant in question to be quenched by cellular antioxidants, such as ascorbate; the BDE for which is $68.5 \mathrm{kcal} / \mathrm{mol}$. A compound would exhibit antioxidant capacity if the BDE of its sacrificial R-H bond falls within the lower portion of this window (Hussain et al., 2003). The weakest bond in BPA is the O-H bond, with a $\mathrm{BDE}$ of $83.9 \mathrm{kcal} / \mathrm{mol}$ ((calculated using the MLM2 method (Wright et al., 2001)), indicating that BPA may be considered a weak antioxidant. It has been noted (Kovacic, 2010) that, as a minor route of xenobiotic metabolism, BPA can be hydroxylated at the ortho position to form a catechol, susceptible to redox cycling and ROS production (Figure 4.1). These factors make BPA unfavorable as a direct antioxidant, suggesting that the antioxidant capacity of BPA may come due to some indirect effect, such as the activation of the Nrfl/2-ARE pathway, as described in this study. Given the reported prooxidant activity of BPA in cultured cells, I hypothesized that BPA might lead to ROS generation by affecting the Nrf1/2-ARE pathway-mediated expression of antioxidant genes. The data, presented here, suggests that BPA activates the Nrfl/2-ARE pathway, as reflected by increased Nrfl transactivation activity and gene expression of ARE target genes ho- 1 and $n q o l$. 


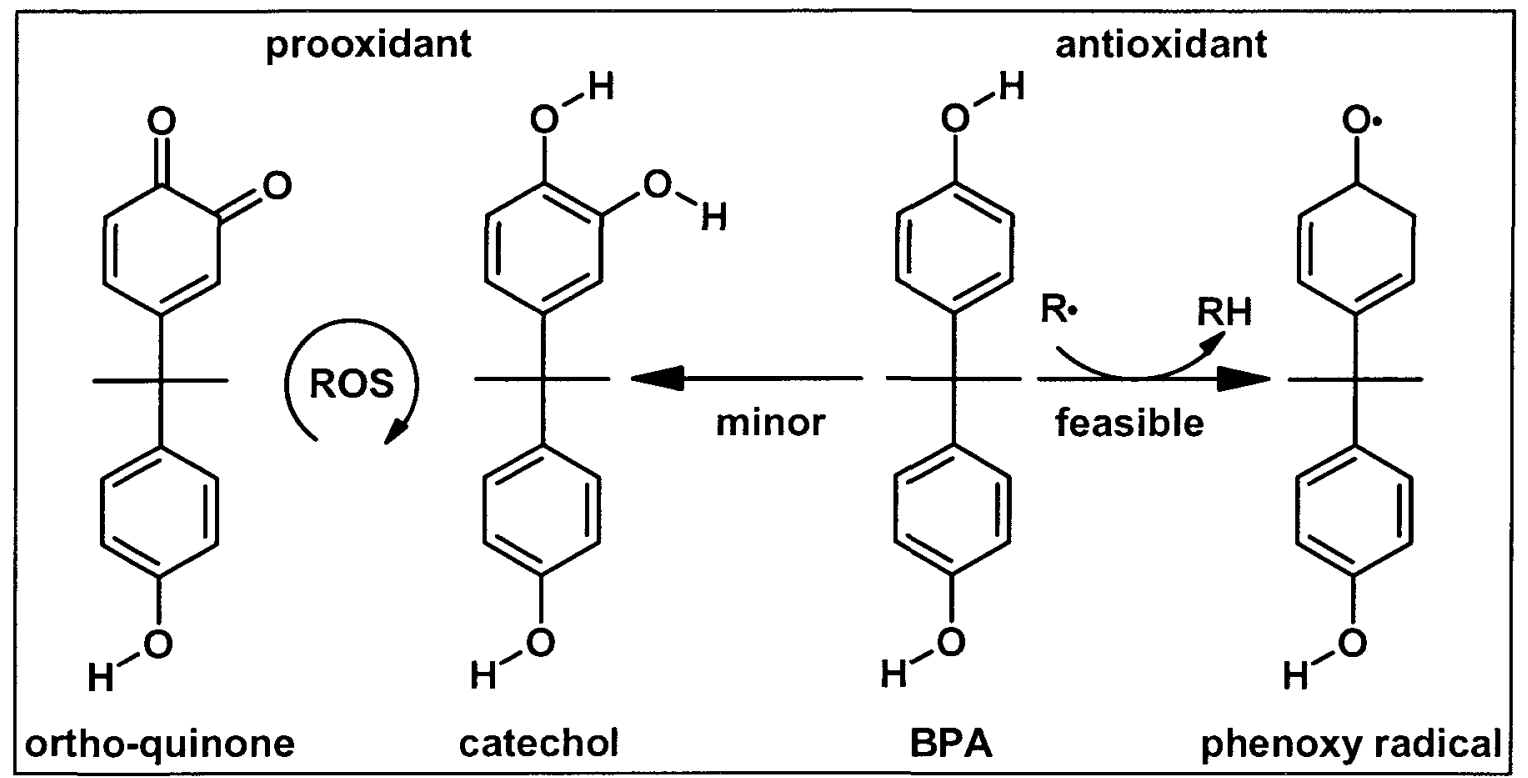

Figure 4.1. Theoretical Pro-oxidant and Antioxidant Properties of BPA. BPA has both pro-oxidant (catechol/quinone redox cycling) and antioxidant ( $\mathrm{H}$ atom donation) potential with the antioxidant potential (predicted $\mathrm{O}-\mathrm{H}$ BDE of $83.9 \mathrm{kcal} / \mathrm{mol}$ ) being considerably distant from the $\mathrm{BDE}$ range of an ideal antioxidant (the lower part of the 68.5-88 kcal/mol window (Hussain et al., 2003)).

\subsection{BPA Promotes Cell Proliferation and Reduces Basal ROS Levels}

First, the effect of BPA on cell viability was examined. The MTT assay results indicated that BPA is not cytotoxic and, instead, promotes cell proliferation at high (50 to $100 \mu \mathrm{M}$ for $24 \mathrm{~h}$ ) concentrations (Figure 4.2A). Furthermore, BPA preferentially accumulated in cytoplasmic and mitochondrial fractions (Figure 4.2B), in accordance with the previous hypothesis that BPA targets mitochondrial membranes (Ooe et al., 2005). BPA treatment reduced the basal rate of ROS production by almost two-fold (Figure 4.2C). This effect 
was already evident after $10 \mathrm{~h}$ (data not shown) and a similar result was obtained in the BPA-treated cultured human fetal lung fibroblasts (hFLF, Chen et al., in preparation). We considered a possibility that the antioxidant-rich serum use in cell culture could mask the production of ROS by BPA. However, the same trend (lowered ROS signal) was noticed even in the cells cultured without serum (data not shown). Similar results were obtained for the GSH/GSSG ratio, an index of intracellular redox balance. Thus, at $100 \mu \mathrm{M}$ BPA, the ratio was about $30 \%$ higher compared to controls (Figure $4.2 \mathrm{D}$ and $4.2 \mathrm{E}$ ). A comparable trend was seen in our protein carbonyl assay, although the difference did not reach statistical significance (Figure 4.2F). These results strongly suggest that BPA induced an adaptive (or compensatory) response leading to more reduced conditions within the cell. 
Figure 4.2. The Effect of BPA on Cell Viability and Intracellular Redox Status. HEK 293A cells were treated with the indicated BPA concentrations for $24 \mathrm{~h}$ and cell viability (A), subcellular BPA content (B), ROS generation (C), total glutathione content (D), the GSH/GSSG ratio (E) and protein carbonylation (F) were assessed as described in the Methods section. Results are presented as means \pm S.E.M. $(n \geq 3)$. An asterisk (*) represents a significant difference from controls (Student's paired ttest, $\mathrm{p}<0.05)$. TOT, total cell lysate; NUC, nuclear fraction; MITO, mitochondrial fraction; CYT, cytoplasmic fraction; ER, endoplasmic reticulum fraction. 


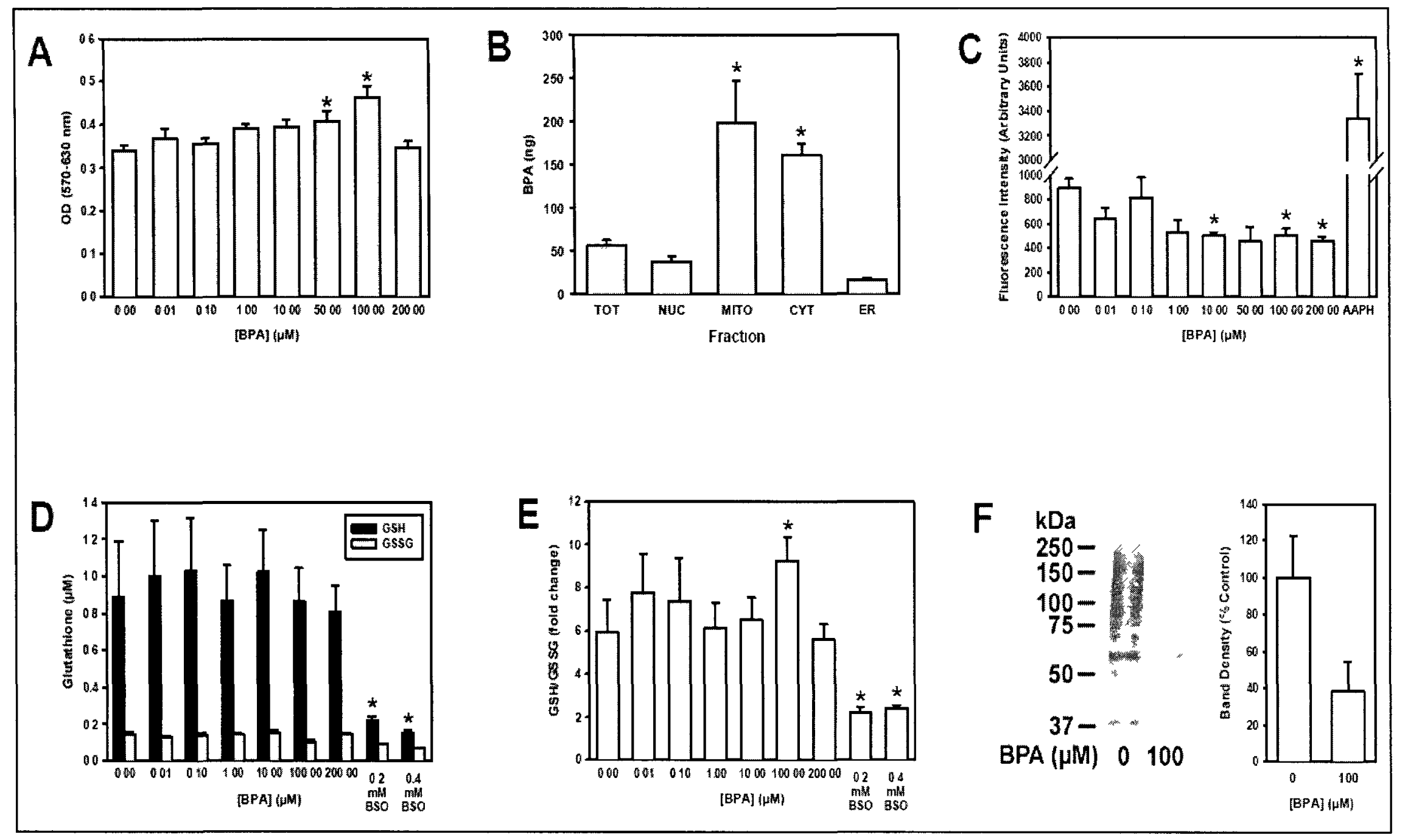




\subsection{BPA Activates Nrf1 and Nrf2 and Alters Protein Binding to the ARE}

To examine protein binding to a typical ARE sequence taken from the $\mathrm{gclm}$ promoter. EMSA analysis was employed. The BPA concentrations of 0,100 and $200 \mu \mathrm{M}$ were selected for the EMSA analysis based on previous results of the ARE-dependent gene expression, which was significantly affected at high concentrations of BPA only as will be discussed in greater detail below. According to Figure 4.3 and the densitometric results not shown here, BPA treatment resulted in essentially no significant change in the protein binding to the labeled ARE probe. The two top bands observed in Figure 4.3 were formed as a result of specific interactions between the ARE and the ARE-binding proteins as confirmed by the addition of excess unlabelled (cold) probe. In this format, the band, whose density is affected by the inclusion of unlabelled probe is considered to be specific to a given DNA sequence because of the competition between unlabelled (cold) and labeled (hot) probes for the binding of ARE-specific proteins, with the later being present in shortage (limiting). While EMSAs are informative in terms of the overall understanding of factors binding to a given DNA sequence, such a binding event can either have a stimulatory or an inhibitory effect on the transcription of downstream genes, depending on the nature of a protein (repressor or activator) bound. In addition, the slight decrease of ARE protein binding by approximately $20 \%$ observed only with $200 \mu \mathrm{M}$ BPA (data not shown), while statistically significant, may be biologically irrelevant in the context of the EMSA approach and therefore a more quantitative assay was required to determine $\mathrm{Nrfl} / 2$-ARE function in the presence of BPA. To gain a more thorough 
and unambigous understanding of the effects of BPA on the Nrfl/2-ARE pathway, we employed a firefly luciferase reporter harboring three AREs from $\beta$-globin enhancer upstream of the luciferase gene (3x ARE-luciferase) or an ARE from the gclc gene, which was co-transfected with pCR3.1 (empty vector), Nrf1-FLAG or Nrf2-FLAG, and the transfected cells were treated with $100 \mu \mathrm{M}$ BPA (Figure 4.4). Nrfl-FLAG activity was significantly higher with $100 \mu \mathrm{M}$ BPA treatment, compared to controls, while Nrf2-FLAG was significantly activated by $200 \mu \mathrm{M}$ BPA treatment. A known Nrf2 inducer, tBHQ (Li et al., 2005), was used as a positive control for Nrf2 induction. The ability of tBHQ to activatet the Nrf2-mediated reporter activity, seen in Figure 4.4B, was used as a positive control for the reporter activity. However, overexpression of a transcription factor in the cells along with a reporter may produce some artefacts as will is discussed in Section 6.2 of this thesis, I wanted to examine the effect of BPA on the expression of the ARE-controlled genes using RT-qPCR. 
Figure 4.3. BPA Alters Protein Binding to the ARE Consensus Sequence Probe.

EMSAs were run on the nuclear lysates from the cells treated with 0,100 and $200 \mu \mathrm{M}$ BPA for 24 hours as described in the Methods section. A lane without protein extract (probe only) and a lane with an excess of unlabelled (cold probe) were used to determine the mobility of the free probe and the specific ARE-protein complexes, respectively. Two representative results of four independent experiments are shown. 


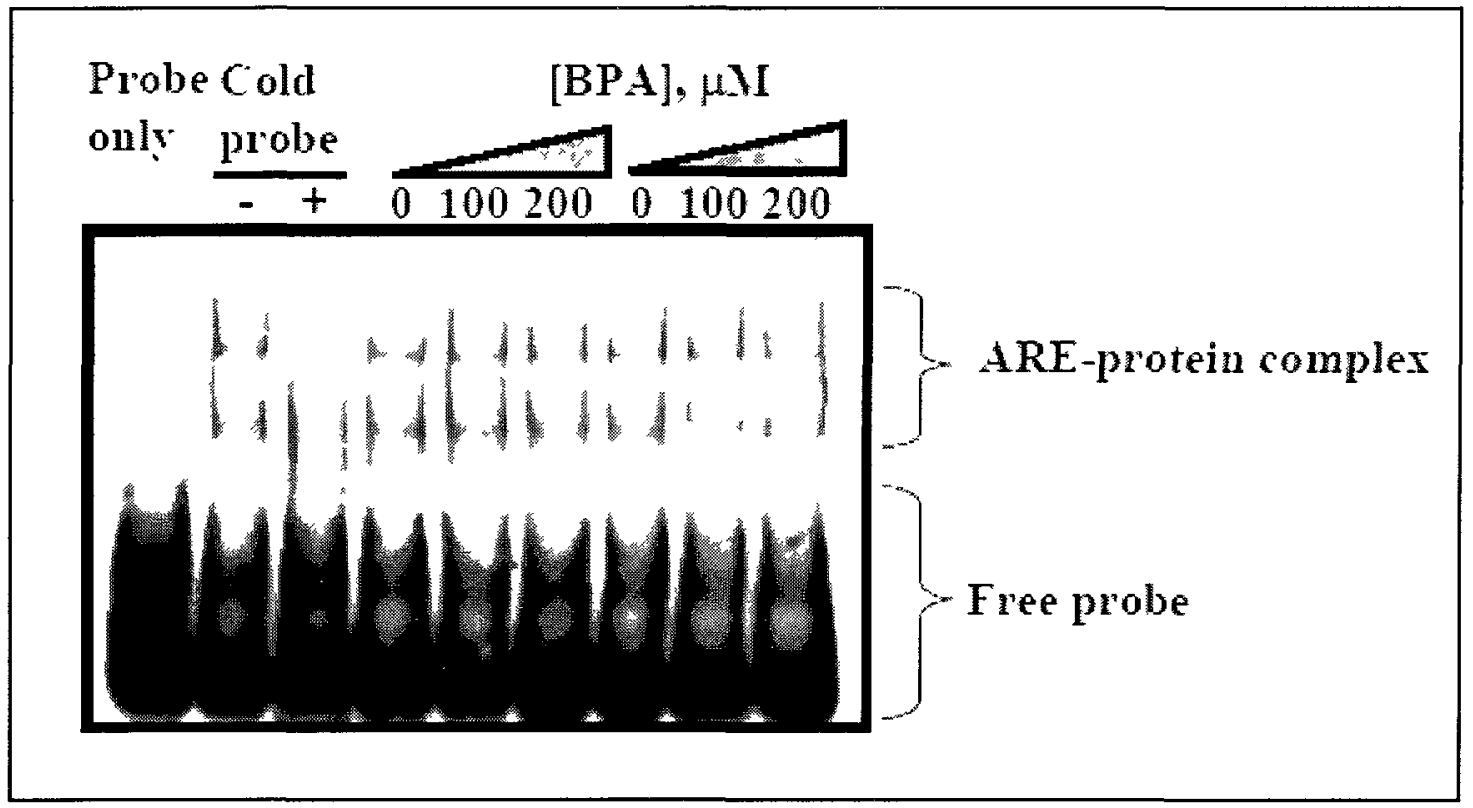


Figure 4.4. BPA Activates Exogenous Nrf1 and Nrf2 in HEK 293 Cells. The results of ARE luciferase reporter assays performed on cells co-transfected with NrflFLAG (A), Nrf2-FLAG (B) or pCR3.1 (transfection control) along with $\beta$-globin $3 X$ ARE-luciferase (A) or gclc ARE-luciferase (B) reporter plasmids and $\beta$-galactosidase (transfection efficiency control) and treated with $0,10,100$ or $200 \mu \mathrm{M}$ BPA doses for 24 h. As a positive control for Nrf2 induction, cells transfected with Nrf2-FLAG or pCR3.1 were treated with dimethylsulfoxide (DMSO, vehicle control) or $100 \mu \mathrm{M}$ tBHQ, a known inducer of the Nrf2-mediated ARE-driven gene expression (Li et al., 2005; Wang and Jaiswal, 2006). The means \pm S.E.M. $(\mathrm{n} \geq 3)$ are shown. Asterisks (*) indicate significant difference from controls (containing dimethylsulfoxide (DMSO); $\mathrm{p}<0.05$, Student's paired t-test). 


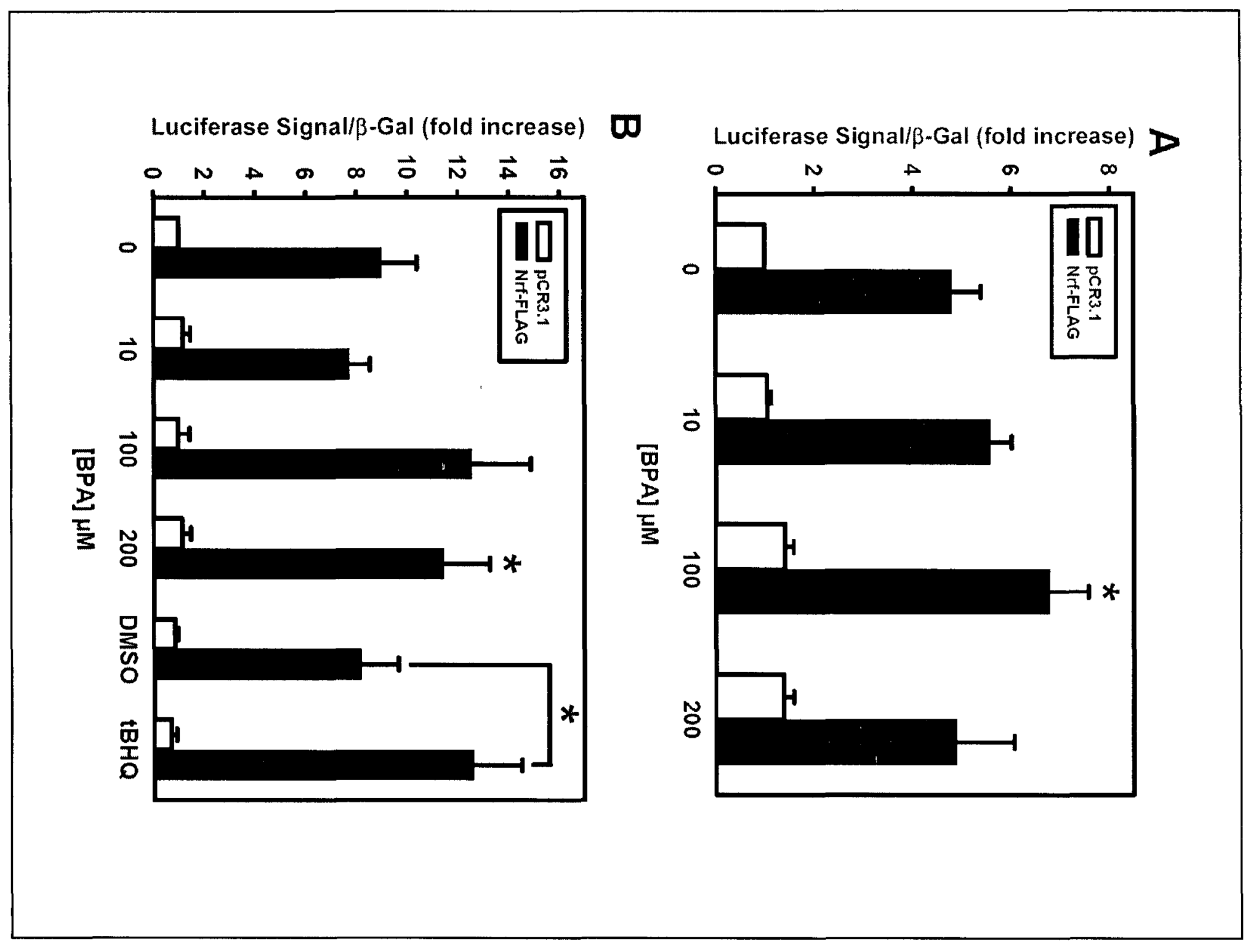




\subsection{BPA Upregulates the Nrf1/2-ARE Pathway-controlled Genes}

Next, the expression of typical ARE-Nrfl/2 target genes upon BPA treatment was analyzed by RT-qPCR. Table 4.1 shows the mRNA expression of $\mathrm{Nrfl}, \mathrm{Nrf2}$, Nqol and $H O-1$ in response to different BPA treatments. The single p-value in Table 4.1 indicates that there was a significant difference between at least 2 doses for a specific gene (by one-way ANOVA). The p-value, however, does not indicate which groups are significantly different from one another. Therefore, a post-hoc Duncan's test was performed to determine which groups differed from one another. Significant differences between all the groups (p-value $\leq 0.05$ ) have been indicated with superscript letters. Different superscript letters between rows indicated fold changes that were significantly different. One hundred $\mu \mathrm{M}$ BPA increased mRNA levels of ho-1 1.39-fold $(\mathrm{p}<0.05$, Table 4.1) and $200 \mu \mathrm{M}$ BPA treatment increased mRNA levels of both ho- 1 and nqol $1.88 \pm 0.48$ - and $2.23 \pm 0.44$-fold, respectively, $(\mathrm{p}<$ 0.05 , Table 4.1) when compared to all other doses. Of note is that $n r f 1$ mRNA levels were unaltered by $100 \mu \mathrm{M}$ BPA treatment, suggesting that Nrfl activation by BPA was probably mediated through post-translational modification of the protein such as ubiquitination or phosphorylation. In fact, the same BPA concentration decreased mRNA level of ubiquitin-conjugating enzyme E2T in cultured hFLF cells (Chen et al., in preparation). Consistent with this is the fact that less ubiquitinated Nrf2 is immunodetectable upon BPA treatment (Figure 4.4A). At $200 \mu \mathrm{M}$ BPA, the expression of both $n r f l$ and $n r f 2$ was upregulated (Table 4.1 ). Very little is currently known about the transcriptional regulation of $n r f l$ and $n r f 2$ genes, other than that, 
under certain circumstances, their expression is regulated in the opposite fashion as is the case for the whole mouse treatment with butylated hydroxytoluene, a known antioxidant and food additive (Chevillard et al., 2010).

\subsection{BPA Downregulates the Protein Expression of Nrf1, Nrf2 and HO-1}

To confirm the possibility that BPA activates the ARE pathway through the alteration of Nrf1 and Nrf2 protein expression, acting on these CNC-bZIP factors directly, immunoblotting was employed to determine the expression levels of the AREpathway regulatory ( $\mathrm{Nrfl}$ and $\mathrm{Nrf2}$ ) and downstream target (Nqo1 and $\mathrm{HO}-1$ ) proteins. The expression of the inactive, glycosylated and ER-bound Nrfl form (p120) was significantly diminished at $100 \mu \mathrm{M}$ BPA treatment without any apparent effect on the active nuclear form of Nrfl (p95) (Figure 4.5). On the other hand, the active and oxidative stress-inducible form of $\mathrm{Nrf} 2$, migrating at approximately 100 $\mathrm{kDa}$ and detectable by our antibody and thought to represent ubiquitinated Nrf2 (Li et $a l ., 2005)$, was about four-fold reduced during BPA treatment. Similarly, the protein expression of HO-1 was about seven-fold reduced by BPA, while the expression of Nqo1 was unchanged. The fact that HO-1 protein expression was down- rather than up-regulated was puzzling and in direct contrast with our PCR results, revealing more than two-fold increase of the ho-1 mRNA level (Table 4.1) and our experiments in hFLF cells, where the HO-1 gene and protein expression levels were increased more than 5- and 18-fold by $100 \mu \mathrm{M}$ BPA treatment, respectively (Chen et al., in - 
Table 4.1 Enhanced Expression of the Nrf1/2-ARE Pathway Regulatory and Target Genes at Different BPA

\section{Concentrations.}

\begin{tabular}{|c|c|c|c|c|c|c|}
\hline \multicolumn{2}{|l|}{ Gene } & \multicolumn{4}{|l|}{ BPA Dose } & \multirow{2}{*}{$\begin{array}{l}\text { p-value } \\
\text { (One-way ANOVA) }\end{array}$} \\
\hline Name & TaqMan assay & $0 \mu \mathrm{M}$ & $10 \mu \mathrm{M}$ & $100 \mu \mathrm{M}$ & $200 \mu \mathrm{M}$ & \\
\hline nqol & $\begin{array}{l}\mathrm{Hs} 00168547 \\
\mathrm{~m} 1\end{array}$ & $1.00 \pm 0.19^{\mathrm{a}}$ & $1.07 \pm 0.16$ & $1.20 \pm 0.20^{\mathrm{a}}$ & $1.88 \pm 0.48^{b}$ & 0.0001 \\
\hline ho-1 & $\begin{array}{l}\mathrm{Hs} 00157965 \\
\mathrm{~m} 1\end{array}$ & $1.00 \pm 0.12^{a}$ & $1.04 \pm 0.25$ & $1.39 \pm 0.28^{b}$ & $2.23 \pm 0.44^{c}$ & $<0.0001$ \\
\hline$n r f 1$ & $\begin{array}{l}\mathrm{Hs} 00231457 \\
\mathrm{~m} 1\end{array}$ & $1.00 \pm 0.16^{\mathrm{a}}$ & $1.08 \pm 0.35$ & $1.18 \pm 0.32^{a}$ & $1.69 \pm 0.23^{b}$ & 0.0015 \\
\hline$n r f 2$ & $\begin{array}{l}\mathrm{Hs} 00975960 \\
\mathrm{~m} 1\end{array}$ & $1.00 \pm 0.07^{\mathrm{a}}$ & $1.14 \pm 0.08$ & $1.21 \pm 0.18^{b}$ & $2.04 \pm 0.22^{c}$ & $<0.0001^{*}$ \\
\hline
\end{tabular}

Relative expression levels of the genes were measured by RT-qPCR analysis and normalized to GAPDH (TaqMan primer Hs 99999905_m1) expression. Values are presented as relative expression means \pm SD $(n=6)$. The single $p$ value in Table 4.1 indicates that there was a significant difference between at least 2 doses for a specific gene (by one-way ANOVA). A post-hoc Duncan's test was performed to determine which groups differed from one another. Significant differences between all the groups ( $\mathrm{p}$-value $\leq 0.05$ ) have been indicated with superscript letters (not indicated in the table). Means in a row not sharing a superscript letter are significantly different, $p<0.05$ (Duncan's Test, one-way ANOVA). * Logarithmically transformed prior to statistical analysis. 
preparation). We thought that the disappearance of the HO-1 monomer could be explained by protein aggregation or dimer formation through covalent bond linkage between the two HO-1 monomers as a result of increased gene and protein expression. To gain a clearer understanding of the behavior of $\mathrm{HO}-1$ protein, we repeated our experiments in HEK 293 cells, paying closer attention to a potential HO1 oligomer formation. As shown in Figure 4.6, we could demonstrate putative HO-1 dimer formation as a result of BPA treatment. Since the reducing and denaturing conditions ( $\beta$-mercaptoethanol, SDS) were used for SDS-PAGE, one would expect that non-covalent associations between HO-1 monomers (aggregates) would be broken. Dimers or oligomers of HO-1 would be only seen in the case of convalent bond formation between two or more subunits. Such a formation of dimers has been observed for a heat-shock protein 25 upon reducing and denaturing SDS-PAGE (HSP25, Ho et al., 2006). More importantly, the HO-1 dimerization and oligomerization has been recently reported (Hwang et al., 2009) under reducing and denaturing SDS-PAGE as well. Whether HO-1 dimerization is driven by partial protein unfolding or by an increase in HO-1 concentration can be tested in a cell-free system utilizing recombinant $\mathrm{HO}-1$, treated with BPA, as has been done previously for citrate synthase that undergoes protein unfolding, aggregation and SDS-PAGEresistant oligomerization in response to oxidative stress in vitro (Chepelev et al., 2009). Dimerization of HO-1 could be important for its activity, which is now thought to be catalytically active as a dimer/oligomer (Hwang et al., 2009). Thus the BPA-mediated dimerization of HO-1 is worth further investigation. 
Figure 4.5. Protein Expression of the Nrf1/2-ARE Pathway Regulatory (Nrf1 and Nrf2) and the Target Proteins (HO-1 and Nqo1) as a Result of BPA Treatment.

HEK 293 cells were treated and with 0 and $100 \mu \mathrm{M}$ BPA for 24 hours and Western blots (A) were performed as described in the Methods Section. For semi-quantitative densitometric analysis (B), band density (the means \pm S.E.M. ( $\mathrm{n} \geq 3$ ) are presented) was normalized to Ponceau S red-stained bands (loading control). Asterisks $\left(^{*}\right)$ indicate significant differences ( $\mathrm{p}<0.05$, Student's paired t-test) from controls. 

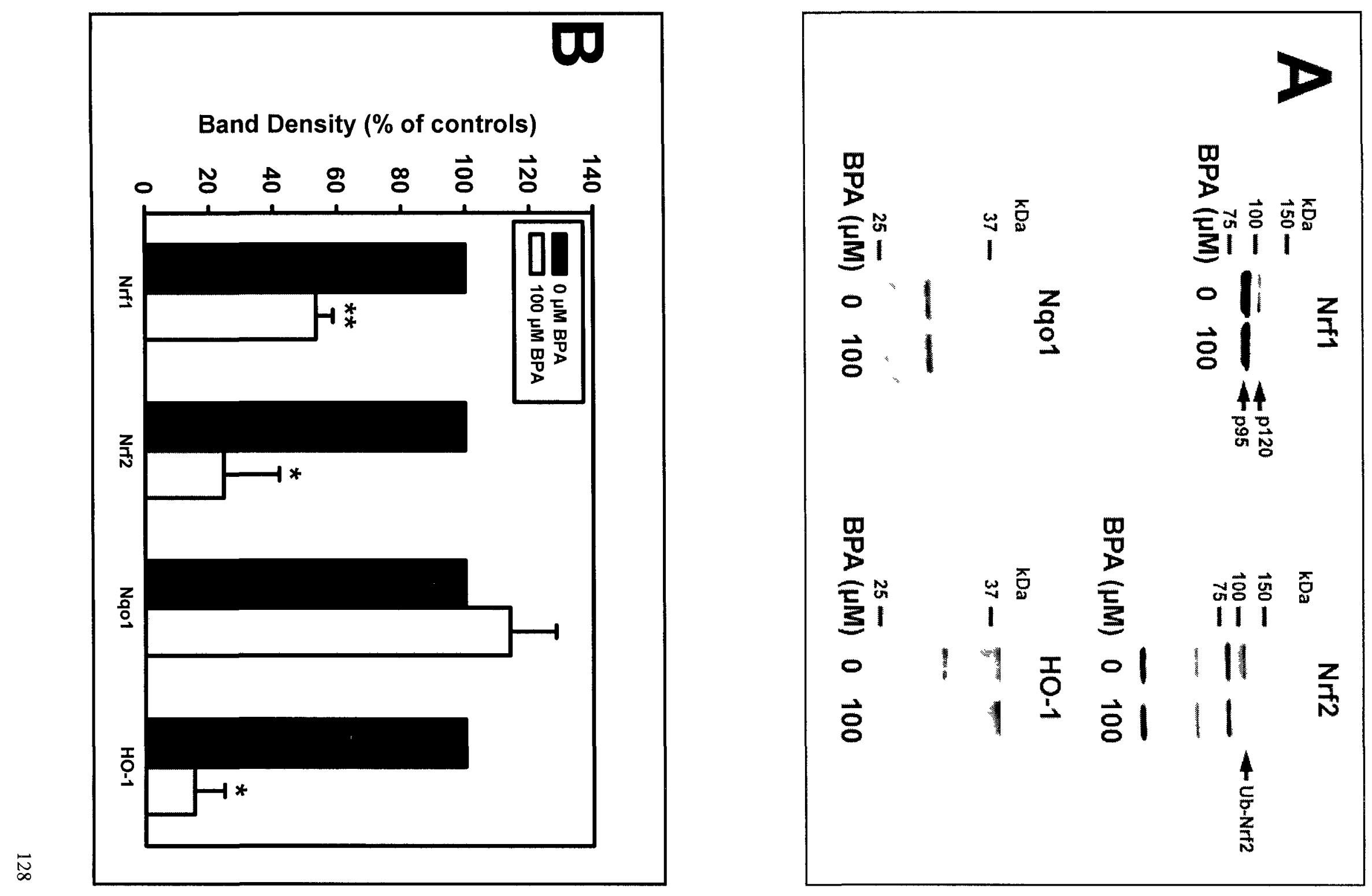


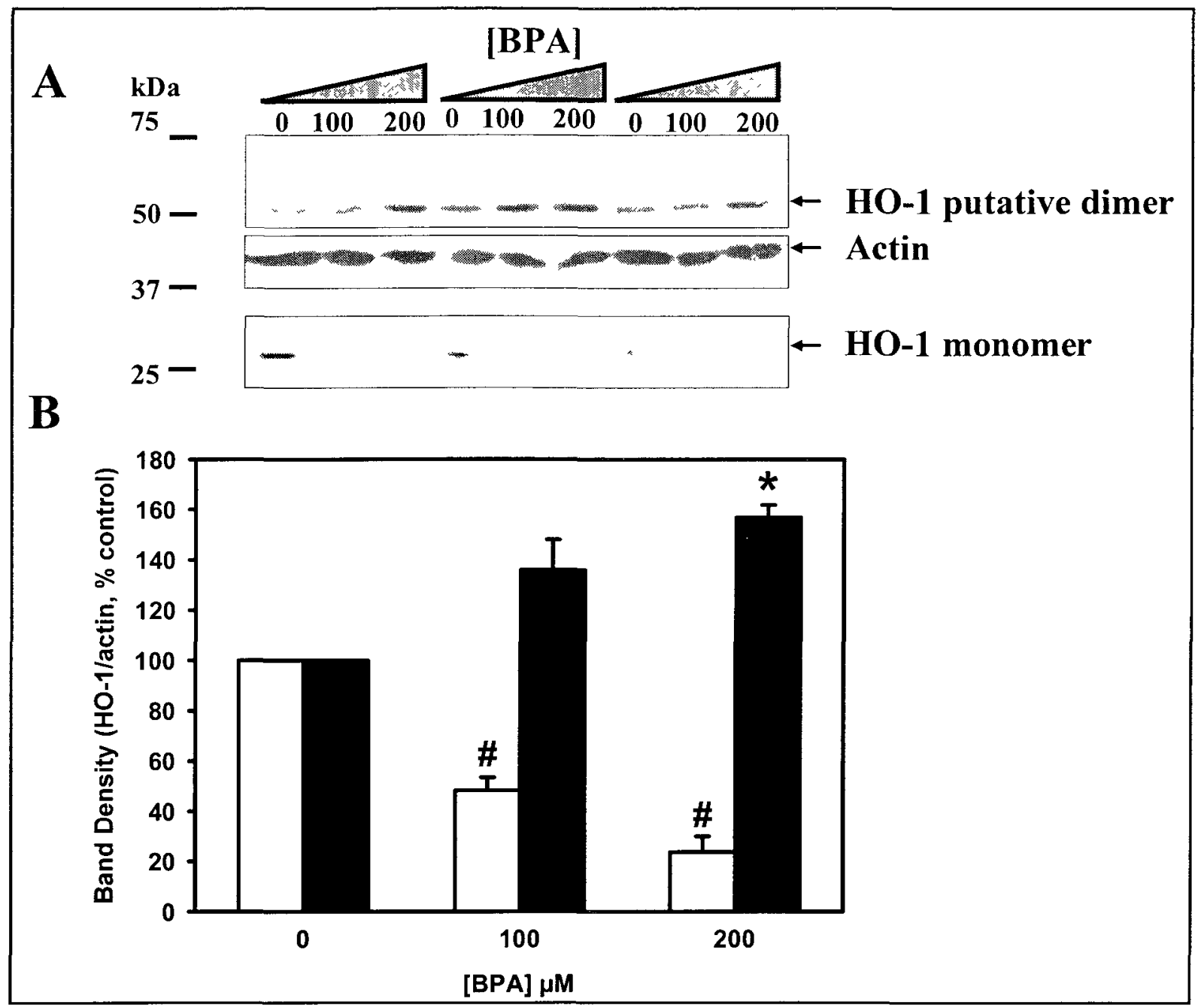

Figure 4.6. HO-1 Putative Dimer Formation as a Result of BPA Treatment. (A) HEK293A cells were subjected to BPA treatment $(0,100$ or $200 \mu \mathrm{M}$ for 24 hours $)$ and the total cellular extract was subjected to immunoblotting as described in the Methods Section. The position of the HO-1 monomer, actin loading control and putative HO-1 dimers are indicated by arrows. (B) Graphical representation of the band densities compared to control $(0 \mu \mathrm{M}$ BPA, means \pm S.E.M. $(n \geq 3))$ is shown. Asterisk (*) and the number signs (\#) indicate statistically significant differences from controls $(p<0.05$ and 0.01 , respectively, as calculated using paired Student's t-test). 


\subsection{The Proposed Model for the Effect of BPA on the Nrf1/2-ARE Pathway}

BPA-induced proliferation of HEK 293 cells, observed in this study, could be explained by the activation of the Nrfl/2-ARE pathway. For instance, constitutive expression of $\mathrm{Nrf} 2$ in human lung carcinoma cells may equip the cells with higher levels of the antioxidant enzymes $\mathrm{HO}-1$ and Nqol, favoring their unrestrained proliferation (Padmanabhan et al., 2006). Given the evidence supporting the activation of the Nrfl/2-ARE pathway presented here, it is plausible to suggest that BPA acted on the pathway to activate the expression of genes involved in cell growth and proliferation. A similar result was reported by $\mathrm{Oh}$ and Lim in Chang liver cells (2008), but the exact mechanisms of BPA-induced cell proliferation and the contributions of Nrf1 and Nrf2 to the process, await further investigation.

The fact that BPA decreased the basal rate of ROS production is in contrast to previous publications (Oh and Lim, 2008; Kabuto et al., 2003; Chtira et al., 2003; Ooe et al., 2005), but in agreement with a similar study in hFLF cells (Chen et al., in preparation). This effect of BPA is in line with the reported antioxidative effects of estrogens, phytoestrogens and polyphenols (Mitchell et al., 1998). It has been shown that these compounds can activate the antioxidant cellular defense system through the MAPK-Nrf2-Keap1 pathway. Similar to our RT-qPCR data, microarray analysis of gene expression in hFLF cells suggested an upregulation of the Nrfl/2-mediated stress response pathways by $100 \mu \mathrm{M}$ BPA (Chen et al., in preparation). Further 
studies of the activities of $\mathrm{Nrfl} / 2$ downstream targets will reveal if they are involved in BPA-specific responses as well as responses to similar phenolics in general.

Halliwell reported some potential artifacts of cell culture studies (Halliwell, 2009). Among these is culturing cells at oxygen concentration about 10-15 times greater than in vivo oxygen levels to which cells are exposed, which could increase the rate of ROS generation. This might explain the fact that HO-1, which is typically stress-inducible, was expressed even under control conditions. If the expression of HO-1, and perhaps other antioxidant enzymes, were increased even prior to BPA application, cells may have been well-buffered against a potential pro-oxidant BPA attack. In addition, BPA acting as antioxidant could counteract elevated ROS levels, creating a more reduced intracellular environment and returning the HO-1 expression back to normal. This scenario, however, is not likely due to the poor predicted antioxidant properties of BPA (see the Section 4.1). Some antioxidant potential of BPA has been previously described, including the inhibition of methacrylate polymerization (Kadoma and Fujisawa, 2000) and prevention of iron-stimulated lipid peroxidation in rats (Kabuto et al., 2003). Given poor predicted antioxidant properties of BPA and the evidence that BPA may act on the Nrf1/2-ARE pathway, the scenario in which BPA could act on the components of the Nrfl/2-ARE pathway to increase the expression of antioxidant enzymes (e.g., glutathione synthesis enzymes, such as gclc and $\mathrm{gclm}$ ) to produce more reduced conditions and relieve hyperoxia-mediated oxidative stress, seems more probable. Another interesting possibility is that BPA could reduce the rate of mitochondrial ROS production, since it has been suggested 
that chemicals with a structure, similar to that of BPA have a tendency to accumulate in the mitochondrial membrane (which is in agreement with Figure $4.2 \mathrm{~B}$ ) because it contains interior hydrophobic proteins and was shown to reduce complex I activity of the mitochondrial electron transport chain (ETC, Ooe et al., 2005). Decreasing the flux through the ETC by lower substrate availability or reduced expression of ETC complex I components is thought to reduce the rate of mitochondrial ROS production (Semenza et al., 2010).

To explain the BPA-provoked activation of Nrfl (Figure 4.4), it would be tempting to speculate that this occurs via deglycosylation of the ER-resident p120 form (since its expression was about 2-fold lower compared to controls) to increase the pool of active p95 form in the nucleus. However, it has not been shown that Nrf1 deglycosylation occurs in vivo. As no change in p95 Nrfl protein expression was observed, BPA could potentially activate Nrf1 by a currently uncharacterized posttranslational modification of Nrfl. This could potentially be phosphorylation since a closely-related family member, $\mathrm{Nrf2}$, is activated by this modification (Numazawa et $a l ., 2003)$. Similarly, Zhang and co-workers (Zhang et al., 2007) suggested that Nrf1 phosphorylation may weaken the association of Nrfl with the ER membrane, stimulating its nuclear translocation. Furthermore, we observed some evidence, supporting that Nrfl-FLAG activity is dependant upon the phosphorylation of Nrfl (Chepelev et al., submitted) and further studies will reveal the significance and the mechanisms by which Nrf1 activity can be regulated through its phosphorylation. 
Despite the increase in ho- 1 gene expression (Table 1), the protein levels of the HO-1 were approximately seven times lower at high $(100 \mu \mathrm{M}) \mathrm{BPA}$ doses (Figure 4.5). The discrepancy between mRNA and protein expression of HO-1 during BPA treatment may be explained by HO-1 dimerization, observed clearly as the appearance of a band with an approximate molecular weight of $55 \mathrm{kDa}$ (Figure 4.6; the molecular weight of the HO-1 monomer is approximately $33 \mathrm{kDa}$ (Yoshiki et al., 2001)) during cell treatment with BPA. This would result in lowered HO-1 monomer protein expression, despite its increased gene expression. Indeed, a recent study has shown that HO-1 exists as dimers and oligomers in the ER, which form through the hydrophobic interactions of the transmembrane segments (TMS, aa 266-285) of HO-1 monomers, of which Trp270 plays a major role (Hwang et al., 2009). Without the TMS-TMS interaction, HO-1 is not anchored to the ER membrane and is a subject of ubiquitination and proteasomal degradation (Hwang et al., 2009; Lin et al., 2008). To the author's best knowledge, this is the first report of a condition, which induced dimerization of HO-1. Given that HO-1 is an enzyme implicated in numoerous clinical conditions and induced by multiple stimuli, the BPA-induced dimerization of HO-1 merits further investigation to disentangle the mechanism(s), responsible for the dimer formation.

In conclusion, it can be envisioned that BPA stimulates the Nrf1/2-ARE pathway as shown by increased mRNA levels of ho-l and nqol (Table 1) and increased transactivation activity of Nrf1-FLAG and Nrf2-FLAG (Figure 4.4). It is conceivable that BPA acted on the mitochondrial ETC to reduce the flow of 
metabolites through the ETC, reducing the rate of mitochondrial ROS generation and altering the intracellular redox status. Figure 4.7 summarizes the current working model for the effect of BPA on the Nrfl/2-ARE pathway. Finally, our study suggests that HEK 293 cells can withstand even high micromolar BPA concentrations, at least within the timeframe studied (0-24 hours), without any significant cytotoxic effects. 


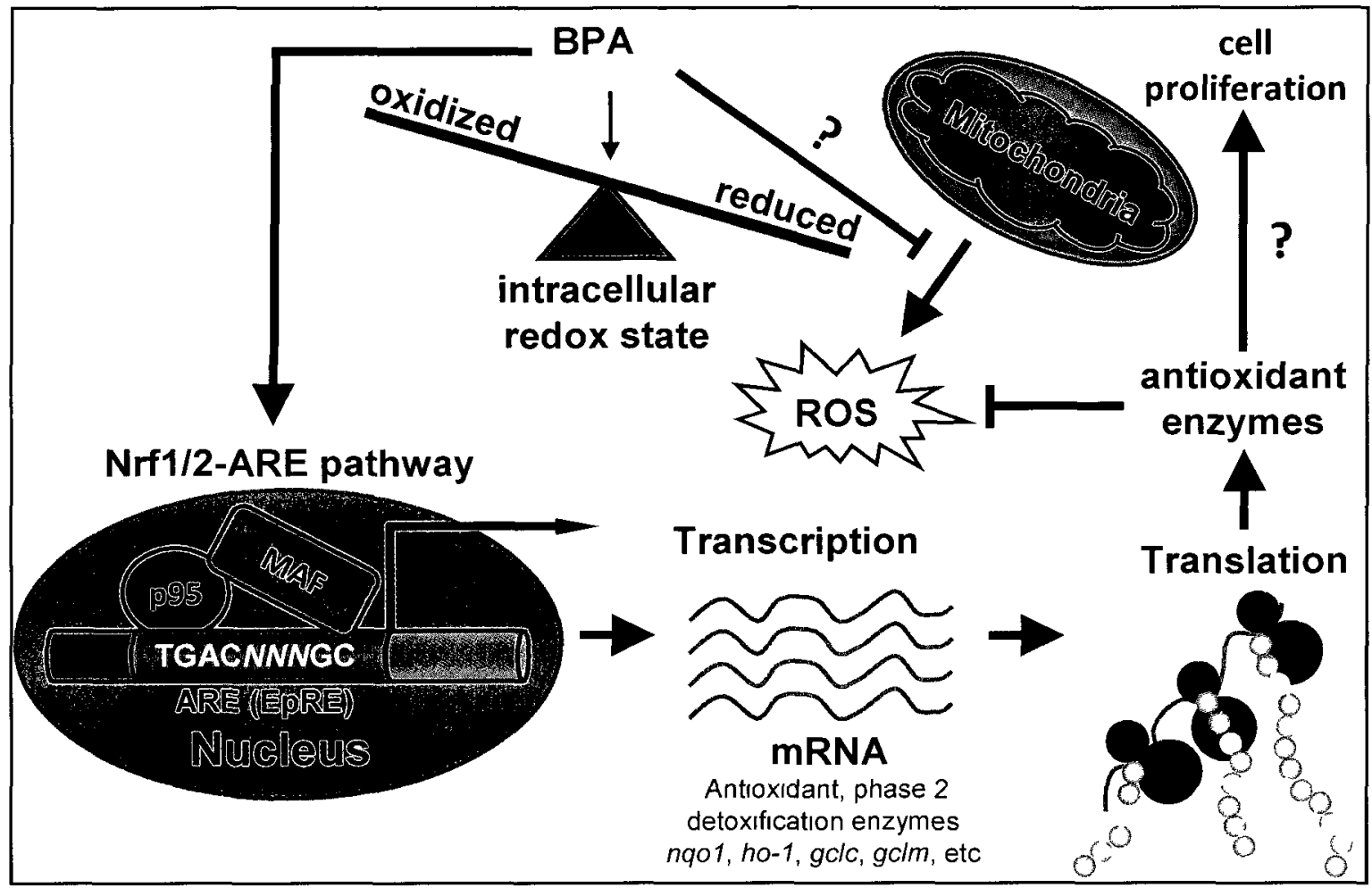

Figure 4.7. The Proposed Model for the BPA Effect on the ARE-Nrf1/2 pathway. BPA treatment results in reduced intracellular conditions in HEK 293 cells (Figure 4.2CE). This could be due to the activation of the ARE-Nrf1/2 pathway (Figure 4.4) such that increased expression of antioxidant enzymes (Table 4.1) could lead to increased cell proliferation (Figure 4.2A). Cytoplasm and mitochondria appear to the main compartments of BPA accumulation (Figure 4.2B) and BPA could also reduce the steady-state ROS levels arising from various intracellular sources such as mitochondria. 
5 Chapter: Nrf1 Regulation in Aging Mice 


\subsection{Aging, Air Particulate Exposure and their Relation to the ROS Generation, Nrf1 and Nrf2}

As has been discussed in the Chapter 1, oxidative stress and ROS contribute to the agerelated complications in humans under stress-related conditions and play a minor role under optimal environmental conditions. Indeed, in laboratory animals in which the expression of the ARE-controlled antioxidant enzymes, such as superoxide 1(SOD1), CAT and GPx, has been abrogated through knockout, significantly higher mortality rates and succeptibility to age-accociated diseases has been shown (Salmon et al., 2010). This concept of increased succeptibility of mammals to environmental toxicants and stress, in general, has led us to consider a model in which both young and old animals were subjected to an environmental stress in order to evaluate the responsiveness of both group of animals towards inducing the expression of the antioxidant genes through the Nrf1/2ARE pathway.

Airborne ultrafine particles, generated by vehicular traffic, are just one example of many widespread environmental stressors. Airborne particles, with a mean diameter of $60 \mathrm{~nm}$, display a pronounced free radical signal as measured by electron paramagnetic

resonance (EPR) spectroscopy, revealing the presence of the carbon-centered free radicals, stable for at least 30 days after collection (Morgan et al., 2011). The inhalation of ambient ultrafine particles, with diameters less than $100 \mathrm{~nm}$, can induce ROS formation and augment oxidative stress in the lung (Donaldson et al., 2005; Nel, 2005; Oberdörster et al., 2005). In the murine phagocyte model of the lung susceptibility to the 
particles, the nanosize group of airborne particles $(<200 \mathrm{~nm})$ induced ROS-mediated damage of the subcellular organelles and increased intracellular ROS production (Xia et al., 2006). Interestingly, nanoparticles are capable of generating ROS even under abiotic conditions (Morgan et al., 2011; Xia et al., 2006). Similarly, the treatment of human lung epithelial cells with combustion-derived fly ash nanoparticles induced ROS generation and augmented the production of glutathione (GSH), a major cellular antioxidant (Diabaté et al., 2011).

Concomitantly with the elevation of basal oxidative stress in aged animals, the expression and activity of antioxidant enzymes, such as glutamylcysteine ligase catalytic (CGLC) and regulatory (GCLM) subunits, is lowered in many aged tissues (see Suh et al., 2004 and references therein). GCLC and GCLM are rate-limiting enzymes of the GSH biosynthesis. It was shown that decreased expression and activity of $\mathrm{Nrf2}$, the master regulator of the inducible antioxidant gene expression, may be responsible for the weakened cellular antioxidant defense in the aged rats (Suh et al., 2004; Shih and Yen, 2006) In contrast, long-lived Ames dwarf mice exhibit elevated expression of antioxidant enzymes, including HO-1 NQO1. More importantly, these animals possess greater inducibility of $\mathrm{Nrf} 2$ and the $\mathrm{Nrf2}$-target genes when challenged with the pro-oxidant diquat (Sun et al., 2011), indicative of their greater capacity to counteract disturbances in their homeostatic intracellular redox environment. It has been noted that long-lived rodents naked mole-rats and white-footed mice have elevated basal Nrf2 expression and GSH content and that Nrf2 homologue SKN-1 in worms and CncC in flies confer these organisms increased longevity (Lewis et al., 2010). Therefore, it has been hypothesized 
that augmented Nrf2 activity is a common feature of the long-lived animals and that $\mathrm{Nrf} 2$ (or its homologues) act as the "gatekeeper of species longevity" (Lewis et al., 2010).

Despite its emerging pro-longevity function, the reasons for declined presence and performance of Nrf2 have not been addressed adequately. As Nrf2 is a tightly regulated protein, multiple mechanisms can be responsible for its age-dependent loss of expression and activity, including somatic loss-of-function mutation of $\mathrm{Nrf} 2$, increased proteolysis of the factor and altered expression and activities of kinases, phosphatases or DNA-binding proteins, leading to reduced transcription of the ARE-target genes, including Nrf2 itself (Sykiotis and Bohmann, 2010). An emerging mechanism of Nrf2 regulation is through the closely-related family member, Nrf1. A short form of Nrf1 (p65Nrfl) is a dominant negative inhibitor of the Nrf2/ARE-mediated gene expression (Wang et al., 2007). In addition, the $\mathrm{Nrfl}$ deficiency resulted in the paradoxical increase of the expression of the Nrf2-target genes in mice liver, which was abolished in Nrf2/Nrf1 double knockout animals (Ohtsuji et al., 2008).

In the current study, the relationship between $\mathrm{Nrf1}$ and $\mathrm{Nrf} 2$ has been explored using Nrf1 RNA interference and Nrf1/Nrf2 overexpression in cultured cells as well as in mice models of aging and airborne particle exposure. I hypothesized that Nrfl can negatively regulate the transactivation activity of Nrf2 in vitro and in vivo and that Nrfl may be differentially expressed in mousemice as a function of age. The data presented here supports a model in which Nrf1 negatively affects the expression of the Nrf2controlled genes. In addition, in the lungs of mice exposed to airborne particles, aging 
diminished Nrf2 expression, and thus its DNA-binding function, as well as the inducibility of the ARE-controlled genes and the increase in the cellular antioxidant capacity. In contrast, the expression of the full-length and short forms of Nrfl was elevated in the lung of the aged- and particle-treated animals. Taken together, these data support a model in which age-dependent change of the homeostatic Nrfl/Nrf2 ratio may impair cellular capacity to counteract deleterious effects of oxidative stressors.

\subsection{Nrf1 Silencing Activates the Expression of the ARE-target Genes}

To investigate potential contribution of Nrfl to the expression of the well-established targets of the Nrf2/ARE-driven gene expression, Nrf1 silencing using interfering RNA oligos (RNAi) was performed in the HBE1 cells. The effect of Nrf1 silencing was examined on the gene expression of $G C L C$ and $G C L M$ using real-time quantitative PCR (real-time qPCR). These are known Nrf2-ARE pathway targets, whose transcription is induced by pro-oxidants 4-hydroxynonenanl and acrolein (Zhang and Forman, 2009). Counter-intuitively, $\mathrm{NrfI}$ silencing resulted in approximately 1.5 -fold increase in the $G C L C$ and $G C L M$ basal expression (Figure 5.1A).

The effect of the Nrfl silencing on the Nrfl protein expression itself was confirmed with Western blotting which revealed that the intensity of Nrfl bands, migrating with apparent molecular weights of 84 and $55 \mathrm{kDa}$, were reduced to about 60 and $70 \%$ of the controls, respectively (Figure 5.1B). Typically, RNAi does not abolish the protein expression of its target mRNA completely and silencing efficiency close to 
$50 \%$ is considered to be acceptable in the literature as was the case with $\mathrm{Nrfl}$ silencing performed by Zhang and co-workers with about 50\% efficiency (2009). It is noteworthy that this banding pattern is different from that obtained using the lysates of HEK293 and WFF2002 cells, which showed distinct bands of 120,95 and $65 \mathrm{kDa}$. The difference is probably due to the use of a different cell line, in which different isoforms of Nrfl may be expressed, in accordance with previous reports on some cell-specific Nrf1 isoforms (as reviewed by Biswass and Chan, 2010). Typically, Nrf1 immunoblotting produces bands with apparent molecular weights of 120 and $95 \mathrm{kDa}$, that are thought to represent glycosylated and unglycosylated Nrfl respectively (Zhang et al., 2007). However, Nrfl can be seen as an $85-\mathrm{kDa}$ polypeptide using other antibodies such as those provided by R\&D Systems (http://www.rndsystems.com/pdf/MAB5306.pdf). Our data is also consistent with previous reports of several Nrfl-specific bands, seen upon Nrfl overexpression, and Nrfl detection by Western blotting including an unstable, presumably, unglycosylated 85-kDa band (Zhang and Hayes, 2010) and a shorter form of endogenous $\mathrm{Nrfl}$, migrating with an apparent molecular weight of $55 \mathrm{kDa}$ and thought to be the Nrfl, proteolytically-cleaved around the Asn/Ser/Thr- (NST)-rich region (Zhang et al., 2009). Both Zhang and co-workers (2009) and the current study utilized the anti-Nrf1 antibodies raised against the $\mathrm{C}$-terminal portions of $\mathrm{Nrf1}$; therefore, the $55-\mathrm{kDa} \mathrm{Nrf1}$ lacks the N-terminal (NTD) domain, keeping the factor in the endoplasmic reticulum(ER)-bound, inactive state. This short Nrf1 form can still act as transcriptional activator as it contains at least two of the three Nrfl transactivation domains (acidic domain (AD)1, NST and AD2 (Zhang et al., 2009; residues 125-324, 325-432 and 433-482 in hNrfl, respectively)). The decrease of the basal $G C L C$ and $G C L M$ expression, observed 
Figure 5.1. NrfI Silencing Activates the Expression of the ARE-target Genes. (A) HBE1 cells were treated with $50 \mathrm{nM}$ scrambled (control) or $N r f l$-specific siRNA (Santa Cruz) for 72 hours and the mRNA expression of GCLC and GCLM was analyzed with the RT-qPCR. The data was normalized to the GAPDH expression and is presented as means \pm S.E.M. $(\mathrm{n}=3)$. Single and double asterisks $\left(*\right.$ and $\left.{ }^{* *}\right)$ indicate statistical significance from the controls with $\mathrm{p}<0.05$ and $\mathrm{p}<0.001$, respectively. (B) The change of Nrfl protein expression upon silencing with either scrambled or Nrfl-specific siRNA and the relative $\mathrm{Nrfl}$ /actin levels was accessed by immunoblotting. The relative density of the Nrfl-specific bands, shown with arrows, is graphed as a representative result of three independent experiments with similar outcome. 

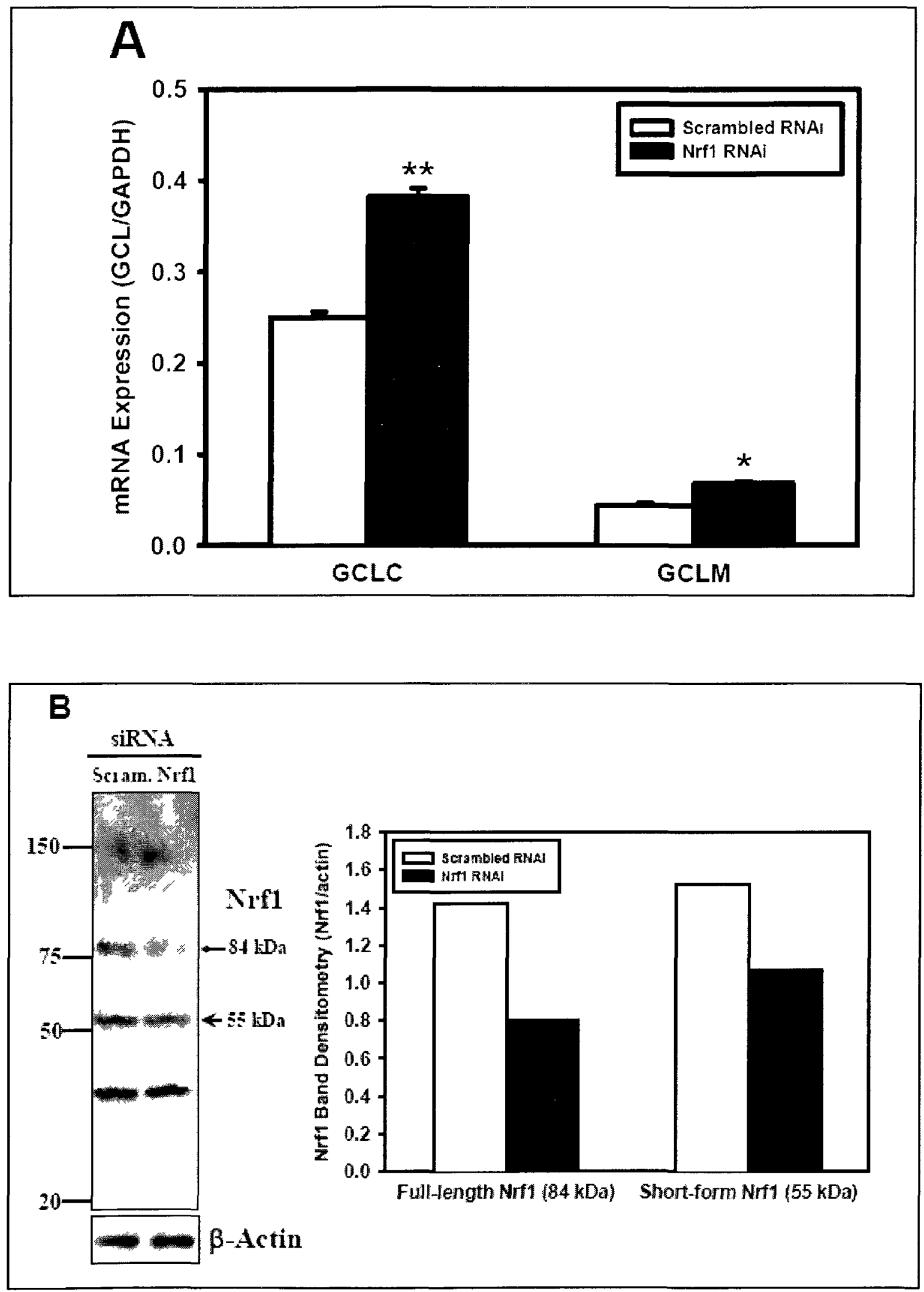
here, is in accord with previous findings (Ohtsuji et al., 2008) in the hepatocytes of Nrfl knockout mice.

\subsection{Exogenous Nrf1 Suppresses Nrf2 Induction by tert-Butylhydroquinone (tBHQ)}

Next, to determine the effect of full-length (p120Nrf1) or short form (p65Nrf1) of Nrf1, ARE-luciferase reporter assays were performed. To this end, HEK293 cells were transfected with GCLC- and GCLM-ARE upstream of the firefly luciferase gene along with p120Nrfl, p65Nrfl and Nrf2. After co-transfection, the cells were treated with a pro-oxidant inducer of the Nrf2-ARE pathway, tBHQ (Wang and Jaiswal, 2006). When both GCLC- and GCLM-ARE luciferase reporters were used, the transactivation activity of p65Nrf1 was approximately 2-3 times lower than that of the full-length Nrf1 (Figure 5.2). This is consistent with the findings of Zhang and co-workers (2009) who showed that the transactivation activity of Nrfl comes mainly from the AD1 and NST domains of the protein, with a minor contribution from the AD2 domain. Naturally, the activity of $\mathrm{p} 65 \mathrm{Nrfl}$ is lower than that of $\mathrm{p} 120 \mathrm{Nrfl}$ as the former lacks the AD1. As expected, Nrf2 transactivation activity was increased by tBHQ treatment with both GCLC- and GCLMARE reporters. With GCLC-ARE, both inducible and basal Nrf2/ARE-driven reporter activity was markedly inhibited by both p65Nrfl (in accord with Wang and co-workers (2007)) and by p120Nrfl (Figure 5.2A). Although the activity of Nrf2 was not as pronounced in GCLM-ARE reporter activation, both forms of Nrf1 were efficient in 
overriding the Nrf2/ARE reporter activity, with no additive effect of the Nrf2 on the reporter activity of both forms of Nrfl observed (Figure 5.2B). These results are consistent with the idea that p65Nrfl (Wang et al., 2007) and p120Nrfl are efficient inhibitors of the Nrf2-ARE driven gene expression and are in good agreement with the stimulatory effect of Nrfl removal on GCLC and GCLM expression (Figure 5.1).

\subsection{The DNA-binding Activities of Nrf1 and Nrf2 Diminish with Age}

Having established the prevailing effect of $\mathrm{Nrfl}$ on the Nrf2/ARE-mediated gene expression in the cultured cell lines, we then delved into examination of the $\mathrm{Nrfl}$ and Nrf2 function in mouse models. The protein binding to the labeled ARE from GCLM was accessed using lung nuclear extracts of the young (6 month) and old (18 month) animals, subjected to the ambient air particles for 10 weeks, at 15 hours per week. As expected from the previous work in aged rats (Suh et al., 2004), aged animal lung nuclear protein

pools contained considerably lower ARE-binding activity (Figure 5.3). Importantly, the treatment with particles reversed the loss of the DNA-binding to AREs in aged animals. The use of antibodies raised against Nrf1 and Nrf2 factors suggested that, not only Nrf2, but also Nrf1 contributed to the ARE binding observed in the nuclear extracts. 
Figure 5.2. The Full-length (p120Nrf1) and 65-kDa (p65Nrf1) Forms of Nrf1 Suppress the Nrf2-mediated Transcription of ARE-controlled Gene Expression. HEK293 cells were transfected with the indicated amounts of the empty vector (pCR3.1), p65Nrfl, p120Nrf1 and Nrf2 along with the luciferase reporter vector with the ARE from GCLC (A) or GCLM (B). A $\beta$-galactosidase plasmid was used as the transfection efficiency control and the luciferase signal was normalized to $\beta$-galactosidase activity. Shown are means \pm S.E.M. of at least three independent experiments $(n \geq 3)$. Asterisks $(*)$ and the number signs (\#) indicate statistically significant differences from controls at the $\mathrm{p}<0.05$ and $\mathrm{p}<0.001$ levels, respectively. 

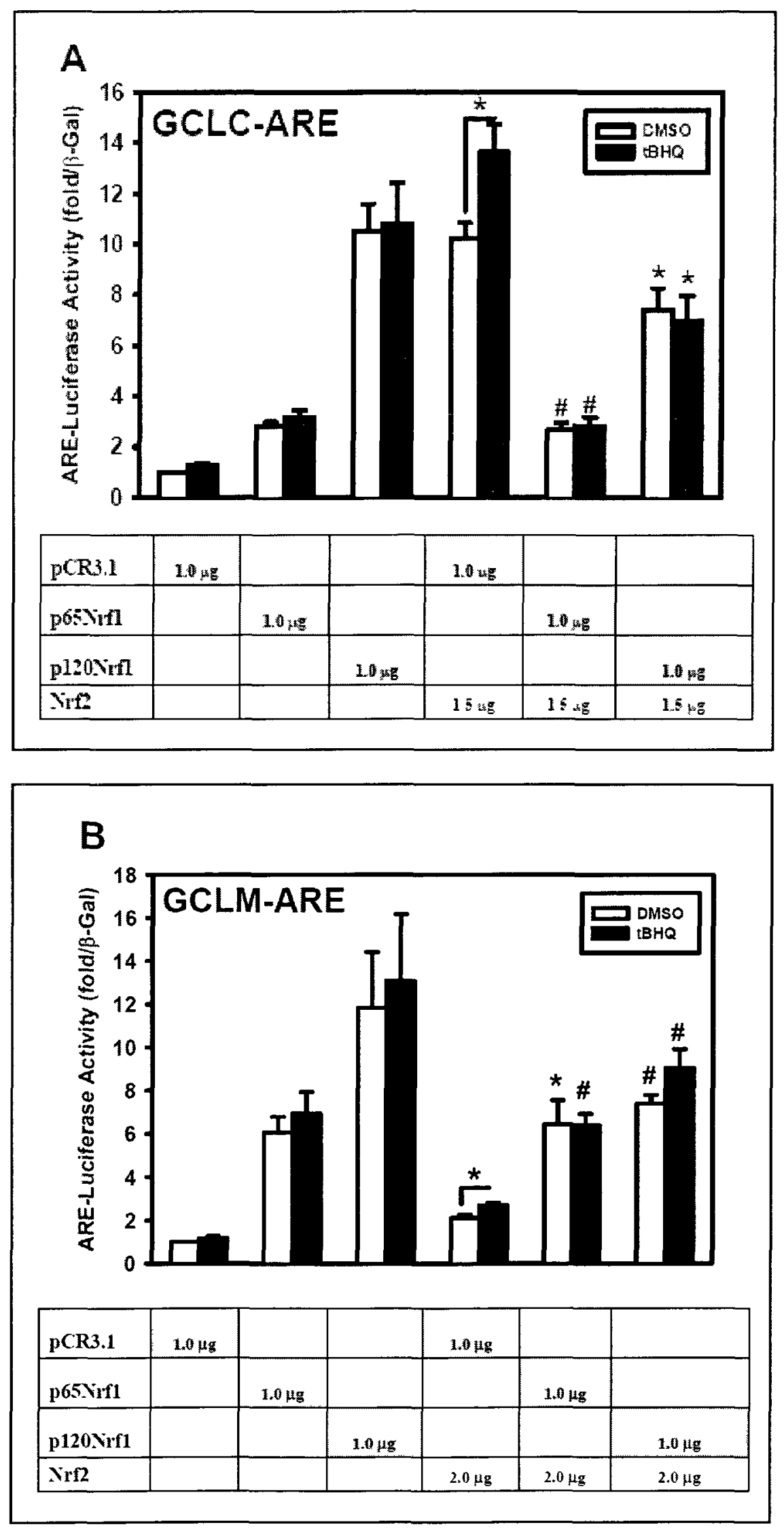


\subsection{Nrf1 and Nrf2 Protein Levels Change in the Opposing Fashion with Age}

The EMSA results, pointing to the contribution of both $\mathrm{Nrf1}$ and $\mathrm{Nrf} 2$ factors to ARE DNA-binding, could be explained by either lowered DNA binding affinity of the factors or their diminished nuclear presence. To investigate the latter possibility, immunoblotting was performed (Figure 5.4). Two different antibodies were used to detect Nrfl and both showed qualitatively similar results. Thus, an increased expression of the band, migrating with an apparent molecular weight of $65 \mathrm{kDa}$, with a similar mobility to that of an exogenously-expressed Nrfl-FLAG positive control, was notable in aged and particletreated animals (Figure 5.4A, top panel). A similar trend was seen for the long form of $\mathrm{Nrfl}$, migrating with an approximate molecular weight of $95 \mathrm{kDa}$ (Figure 5.4A, middle panel), and for the Nrfl band detectable with the C-terminal antibody and migrating as a 55-kDa polypeptide (Figure 5.4A, bottom panel). On the other hand, the nuclear levels of Nrf2 were decreased in aged mice, but were increased by the particulate exposure in both young and aged animals, with the effect being more pronounced in the 6-month mice (Figure 5.4C and D). These results indicate that: i) aging regulates the Nrf1 and Nrf2 levels in the opposite direction, reminiscent of the Yin-Yang type of pattern; ii) the administration of exhaust particulate matter to mice invokes increased expression of both Nrf1 and Nrf2 and iii) that the stimulatory effects of particulate exposure on the Nrf1 and Nrf2 protein expression is more pronounced in the young animals.

\subsection{The Inducibility of the Nrf1/2-ARE-controlled Genes and the Cellular Antioxidant Defence Capacity is Impaired with Age}


Finally, the expression of an ARE-target gene NQO1, as well as the cellular capacity of mouse lung to mount an efficient antioxidant defence upon the exposure to the airborne particulate matter, was examined using real-time qPCR and oxygen radical absorbance capacity (ORAC) assay. The mRNA expression of NQOI, an established subject of the Nrf1/2-ARE regulation (Dinkova-Kostova and Talalay, 2010), was markedly increased by particulate treatment of the young, but not old, mice (Figure 5.5A). A similar trend was noticeable with the ORAC assay, showing that only young animals could increase their total antioxidant defence (oxygen radical scavenging) capacity when challenged with airborne particles (Figure 5.5B). These results are indicative of impaired ability of the Nrf2-ARE pathway to elicit an appropriate response to the pro-oxidant stimulus.

\subsection{The Proposed Model for the Effect of Aging and Particulate Exposure on the Nrf1 and Nrf2 Function in Mice}

The current study demonstrates, for the first time, that both full-length and short form of Nrfl have an inhibitory effect on the ARE-driven gene expression, mediated by $\mathrm{Nrf2}$, as evidenced by the experiments with $\mathrm{Nrf1}$ silencing and $\mathrm{Nrf1} / \mathrm{Nrf} 2$ overexpression in cultured human cells. Thus, the removal of $N r f 1$ by RNA interference increased the gene expression of GCLC and GCLM, in accord with the previous report from the Yamamoto lab (Ohtsuji et al., 2008), examining hepatocyte-specific Nrfl knockout mice model. In

that study, Nrfl deficiency increased the expression of multiple ARE/Nrf2-controlled genes, including $G C L C, N Q O 1$ and $H O-1$. More importantly, this stimulatory effect of 
the $N r f 1$ disruption was completely abolished in the livers of the Nrf1:Nrf2 double knockout mice (Ohtsuji et al., 2008), suggesting that Nrfl may act as an inhibitor of the basal Nrf2-driven gene expression, in good agreement with our data. Similarly, the cotransfection of the HEK293 cells with p65Nrf1 or p120Nrfl together with Nrf2 completely abrogated the Nrf2-driven GCLC-ARE-luciferase reporter activity and fully overrode both basal and inducible GCLM-ARE-driven luciferase expression, confirming our hypothesis that Nrf1 has an inhibitory effect on the Nrf2 transactivation activity. Mechanistically, the dominance of Nrfl over Nrf2 may be attributed to the differences in their transactivation domains, such that only one and not the other may recruit all the necessary components of the basal transcription machinery to transcribe a given gene. This, apparently, is the case for the metallothionein $1(M T 1)$, whose expression is controlled exclusively by Nrfl and not Nrf2, despite the fact that both factors bind the MTI-ARE with equal binding affinity (Ohstuji et al., 2008). Another possibility is that the inhibitory effect of Nrfl, revealed by the $\mathrm{Nrfl}$ silencing and overexpression is due to the short form of Nrfl, p65Nrfl, which lacks the AD1 transactivation domain and may act as a repressor of $\mathrm{Nrf2}$ by competing with $\mathrm{Nrf2}$ for DNA-binding to the ARE (Wang et al., 2007). The existence of such a negative control by p65Nrfl is hypothesized to be in place to avoid the unrestrained expression of ARE target genes, which can be carcinogenic (Wang et al., 2007). Our luciferase assay results with p65Nrfl confirmed the data from the Chan lab (Wang et al., 2007); in addition, we have shown that not only p65Nrfl, but also p120Nrfl, is capable of achieving marked reduction of both Nrf2 basal and $\mathrm{tBHQ}$-inducible transactivation activity. 
These in vitro results provided an invaluable starting point in the attempt to study the existence of potential Nrf1/Nrf2 interaction in mouse models of pro-oxidant exposure. Previously, it was reported that airborne particles from the vehicular traffic contained stable, carbon-centered radicals and the particulate treatment evoked pronounced inflammatory responses in mice and glutamatergic neurotoxicity in rat brain slices and cultured primary neurons (Morgan et al., 2011). Taking these findings into account, it was expected that mouse lung is a good in vivo model of the Nrf2-ARE pathway responsiveness to the airborne traffic particles, according to the hierarchical oxidative stress model (Li et al., 2010). According to this model, explaining the pro-oxidant effects of diesel exhaust particles, the Nrf2-ARE pathway constitutes the first tier of cellular defense and it is activated at the stress levels below those needed to activate proinflammatory responses, the second cellular defense against exhaust particles ( $\mathrm{Li}$ et al., 2010). Therefore, given the report (Morgan et al., 2011) of certain inflammatory changes as a result of the particle treatment of mice in the brain, an organ which is not expected to be in such a direct contact with the particles as the lung is, it was reasonable to expect that that the treatment would affect the Nrf2-ARE pathway, allowing us to dissect potential contribution of $\mathrm{Nrfl}$ and $\mathrm{Nrf2}$ to the aging and the cellular antioxidant defense against the particles.

The notable effects of the particle treatments compared to the controls (air) included: i) restoration of the age-impaired Nrfl- and Nrf2-ARE binding, ii) increased protein expression of $\mathrm{Nrf} 2$, more pronounced in the young animals, iii) increased protein expression of Nrfl, iv) increased NQOI mRNA content in young mice and v) increased 
oxygen radical scavenging capacity in the samples from young, but not old animals. All these are indicative of the induction of the Nrfl/2-ARE pathway to counter the deleterious effects of the airborne particles, albeit such an effect was more pronounced in younger mice. It can be envisioned that, due to the accumulation of the oxidative damage over time (Sohal an Weindruch, 1996), the Nrf1/2-ARE pathway in aged animals can already be in its induced state, as suggested by the fact that "basal" NQO1 (Figure 5.5A) and HO-1 (data not shown) mRNA levels and antioxidant capacity measured by the ORAC assay in aged animals were already higher compared to the young subjects even without particulate matter treatment. The prominent effects of aging included: i) the decrease in the Nrf1/2-ARE binding, ii) decreased protein expression of Nrf2, iii) increased NQO1 mRNA expression, and iv) increased oxygen radical scavenging capacity. Effects i) and ii) are indicative of the impairment of the Nrf2-ARE pathway, such that diminished ARE binding could be related to the lowered $\mathrm{Nrf} 2$ content in agreement with previous study in aged rats (Suh et al., 2004). However, it is noteworthy that the Nrfl and Nrf2 protein expression changed with age in the opposing fashion, such that the Nrf2/Nrfl ratio decreases with age. It is currently unclear what regulatory event(s) is(are) responsible for the paradoxical reduction of the Nrf2 protein content in aged mice despite the increased oxidative environment and thus, greater demand for the enhancement of the cellular antioxidant gene expression. One possibility is that the redox-sensing capacity of the INrf2 (also known as Keap1), an E3 ubiquitin ligase adaptor, facilitating the ubiquitination of $\mathrm{Nrf2}$ and its rapid proteasomal degradation, is impaired with age (Suh et al., 2004). However, studies in the cultured mouse fibroblasts showed that subjecting cells to more oxidizing extracellular redox potential $(-46$ and 0 
$\mathrm{mV}$ ), to approximate the age-dependent redox changes, still induced Nrf2 translocation from the cytoplasm to the nucleus (Imhoff and Nansen, 2009), suggesting that alteration of the cellular redox status alone fails to explain the decline of Nrf2 expression with age. Alternatively, an INrf2-independent mechanism of Nrf2 control is known, involving the phosphorylation of some Ser residues of the Neh6 domain of Nrf2 by glycogen synthase

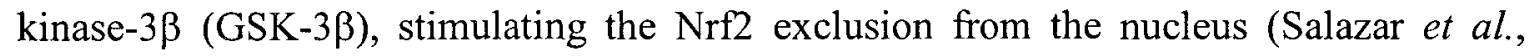
2006). The GSK-3 $\beta$ activity is, itself, inhibited by the Akt-mediated phosphorylation at the Ser9 residue and Akt inhibition stimulates nuclear translocation of Nrf2 (van Weeren et al., 1998; Rojo et al., 2008). A more recent work reported that the levels of the phosphorylated GSK-3 $\beta$ and phosphorylated (inactive) Akt decreased with age in the liver of mice models of accelerated aging (Tomobe et al., 2011), suggesting that the GSK-3 $\beta$ activation could be responsible for the reduced nuclear Nrf2 content.

Unlike its well-stidied Nrf2 CNC-bZIP family member, the mechanisms of Nrfl control remain largely enigmatic. The full-length Nrfl shows a double band on the Western blot, corresponding to 120 - and $95-\mathrm{kDa}$ proteins ( $120 \mathrm{Nrf1}$ and $\mathrm{p} 95 \mathrm{Nrf1}$, respectively). The p95Nrf1 represents the non- or unglycosylated Nrf1, while the p120Nrf1 is the glycosylated form (Zhang et al., 2007). The factor is localized primarily to the ER and nuclear envelope (Zhang et al., 2009). In addition, the ER redox status and Nrfl glycosylation status are hypothesized to cause Nrfl relocation to the nucleus from the ER (Zhang et al., 2009). Nrfl may also be activated by the proteasomal cleavage of its N-terminus to remove the inhibitory NTD, producing smaller, more active forms of Nrf1 (Schultz et al., 2010; Chepelev et al., unpublished). The anchoring of Nrf1 to the 
ER by the NTD domain is thought to be weakened by the stimulation of Nrfl Tyr phosphorylation by pro-oxidants, stimulating the nuclear import of the otherwise ERbound Nrfl (Zhang et al., 2009). In addition, there is a short form of Nrfl, migrating as a 65-kDa protein (p65Nrfl) on the SDS-PAGE, presumably arising from the Nrfl translation from an internal start codon (Chan et al., 1993) The p65Nrfl acts as a dominant negative inhibitor of Nrf2-mediated, ARE-driven luciferase reporter activity, competing with Nrf2 for the DNA binding (Wang et al., 2007). The upstream signalling factors, regulating Nrfl, have not been elucidated in great detail. The regulation of Nrfl by the proteasome has recently been described (Steffen et al., 2010; Radhakrishnan et al., 2010). Thus, the ER-bound Nrf1 appears to be controlled by the ER-associated protein degradation (ERAD) system, involving ubiquitin-dependent proteasomal degradation of its protein substrates (Steffen et al., 2010). To our best knowledge, there has not been a single report of the induction of the Nrf1 protein expression by treatments other than protesomal inhibition (Zhang et al., 2009; Steffen et al., 2010; Radhakrishnan et al., 2010), except for one study, revealing arsenic-induced stabilization of the full-length Nrf1 (Zhao et al., 2011). It is known that aging is associated with the proteasomal dysfunction, partly due to the accumulation of oxidized proteins and protein aggregates that could overload the proteasome-dependent functions (Grimm et al., 2011). Therefore, an attractive hypothesis is that the age-dependent Nrfl stabilization observed here could be due to impaired Nrfl degradation by the proteasome. As Nrf2 is also a subject of the proteasomal degradation, albeit by a different proteasomal-degradation pathway than $\mathrm{Nrfl}$, the differences in Nrfl and Nrf2 age-dependent stability can reflect differential changes in sensitivity of the two proteasomal clearance pathways during aging. 
Considering the inhibitory effect of $\mathrm{Nrfl}$ on the Nrf2 transactivation function (Figures 5.1 and 5.2), it seems reasonable to hypothesize that the age-associated decline in the homeostatic $[\mathrm{Nrf} 2] /[\mathrm{Nrfl}]$ ratio may be the underlying cause for the decreased responsiveness of the Nrf2-ARE pathway to the stimulation with the airborne particles. Our working model for the combined effect of the particulate exposure and aging on the ARE pathway is summarized in Figure 5.6. In conclusion, this is the first study, describing the Yin-Yang pattern of the Nrf1/Nrf2 protein expression associated with aging. Future studies will reveal the exact mechanisms responsible for Nrfl age- and airborne exhaust particle-dependent stabilization with the impairment of the ERAD pathway being one possible hypothesis. More importantly, the analysis of Nrf1 and Nrf2bound co-activators or co-repressors by such techniques as immunoprecipitation and/or mass spectrometry is expected to yield invaluable clues to understand the inhibitory effect of Nrfl on the Nrf2/ARE-driven gene expression. 
Figure 5.3 Aging is Accompanied by Decreased Protein Binding to the ARE. Mouse lung nuclear protein extract $(7.5 \mu \mathrm{g}$ per lane) was incubated with the biotinylated $G C L M$ $A R E$ probe and the protein binding to the ARE was examined using horseradish peroxidase-conjugated streptavidin and chemiluminescence. The specificity of the doublet band, representing specific ARE-protein complexes, was deduced using free probe only and by the addition of an excess of unlabelled (cold) probe to the reactions. The involvement of Nrfl and Nrf2 in the ARE-protein complex formation was revealed

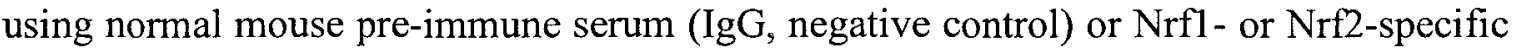
antibodies. P.M. and N.E. designate the samples from particulate matter-treated animals, and non-exposed controls, respectively. For the antibody-supershift experiments, $20 \mu \mathrm{g}$ of protein extract from 6-month, air-treated animals per lane was used. The representative results of three independent experiments are shown. 


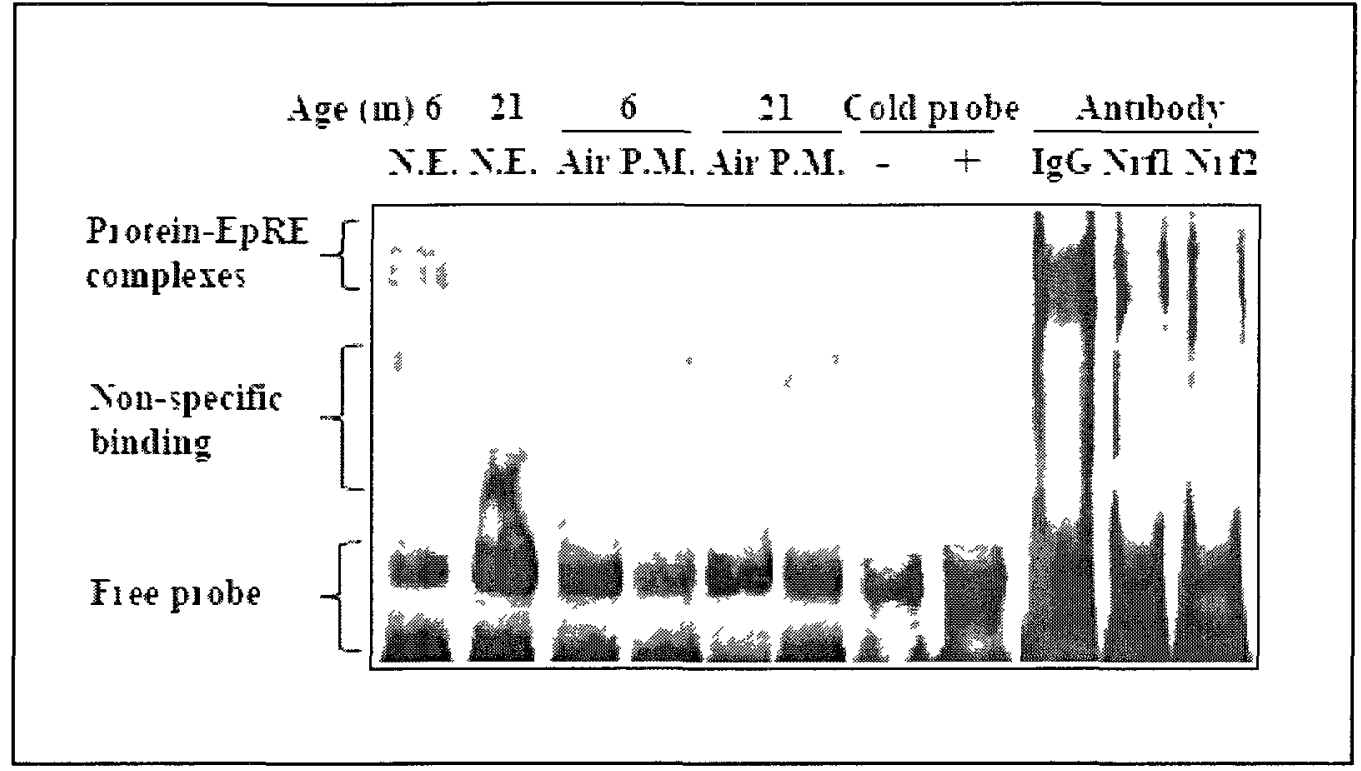


Figure 5.4. Nrf1 and Nrf2 Protein Levels Change in the Opposing Fashion with Age. The protein expression of Nrfl (A) and Nrf2 (C) in the lung of young and old mice, treated with airborne particles, was studied with Western blotting. The lysates of the HEK293 cells, transfected with an empty vector (pCR3.1) or Nrf1-FLAG were used as a positive control for Nrfl expression (A). Graphical determination of Nrf1 (B) and Nrf2 (D) densitometry with respect to loading controls is presented. Data are presented as means \pm S.E.M. and results shown are representative of at least three $(n \geq 3)$ independent experiments. 

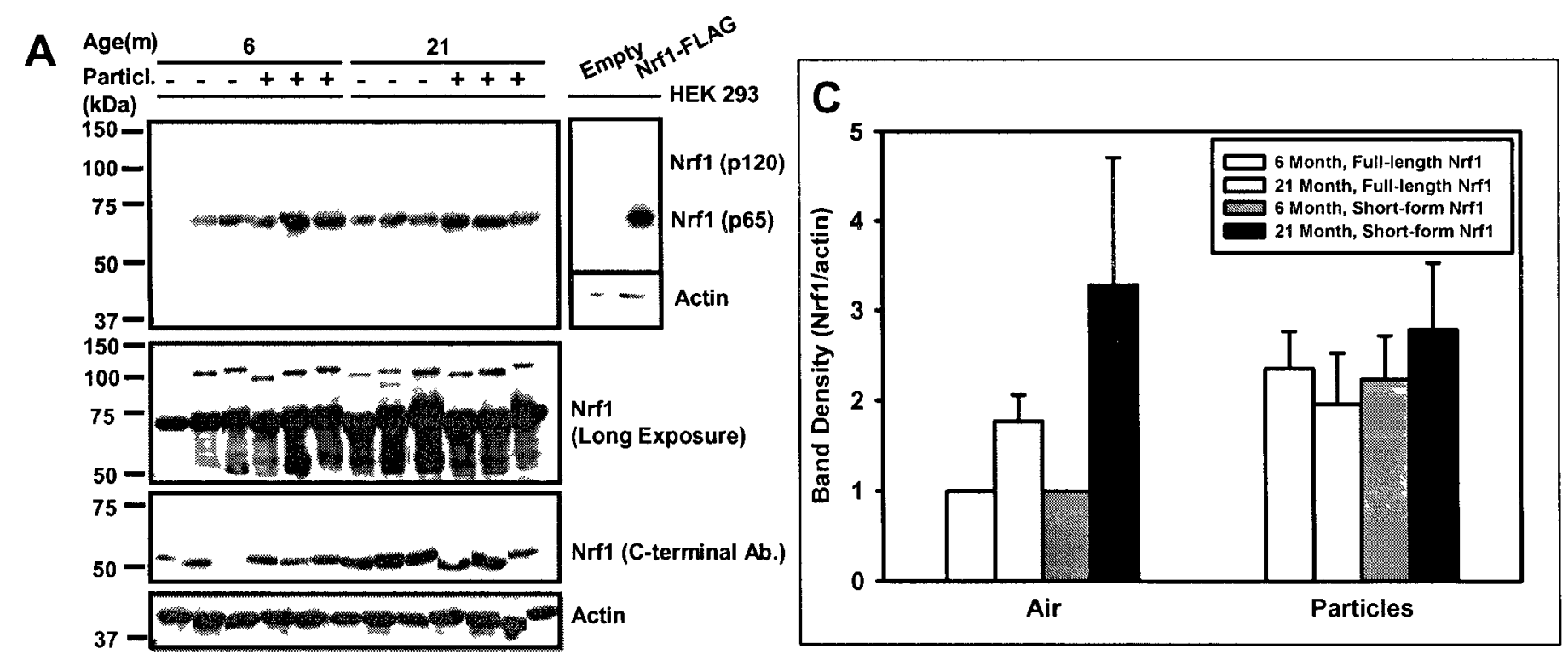

B

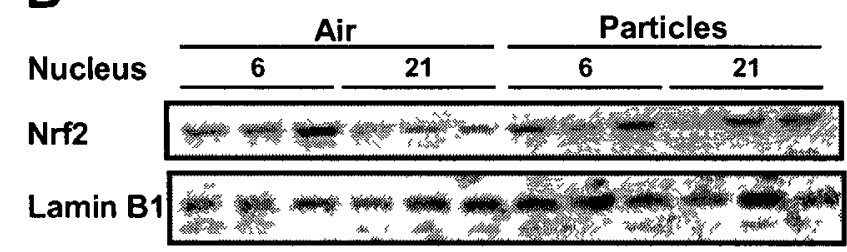

Cytosol

Nrf2

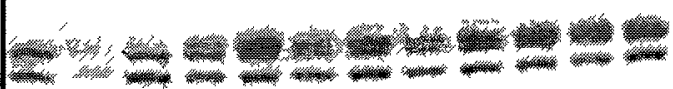

$\beta$-Actin

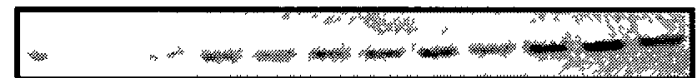

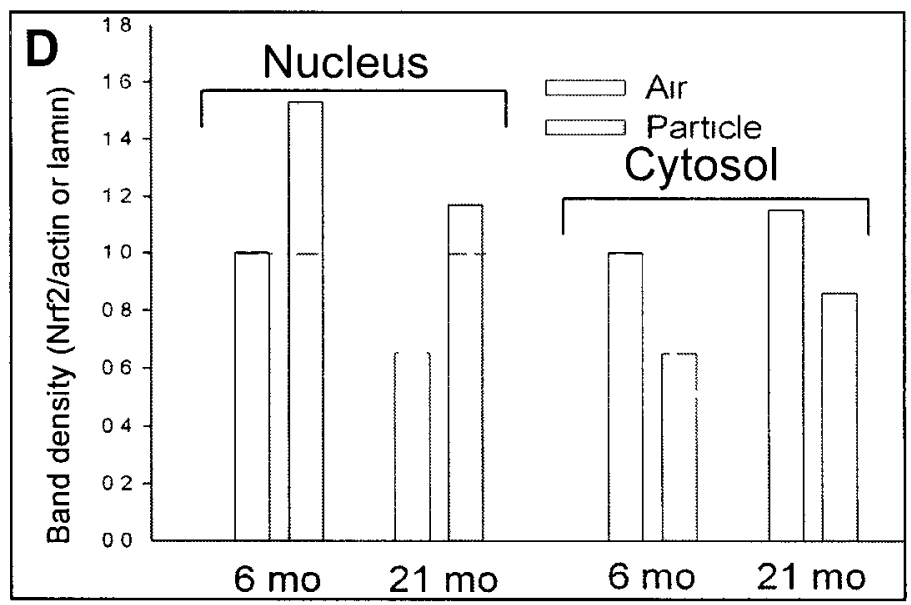


Figure 5.5. Age-dependent Decline in the Inducibility of the Nrf1/2-controlled Gene Expression and Oxygen Radical Scavenging Capacity. The lung samples of young and old mice, subjected to the ambient air or particulate exposure, were analyzed for the expression of the ARE-regulated enzyme $N Q O 1$ by the real-time qPCR (A) and for their ability to scavenge the peroxyl and alkoxyl radicals generated by AAPH in the ORAC assay format (B). Data is presented as means \pm S.E.M. of the relative mRNA expression, normalized to $G A P D H$, and to the controls (A) or as $\mu$ moles of Trolox equivalents per $\mu \mathrm{g}$ of cytoplasmic protein (B). Asterisks (*) indicate significant difference from the corresponding controls $(\mathrm{p}<0.05, \mathrm{n} \geq 3)$. 
A

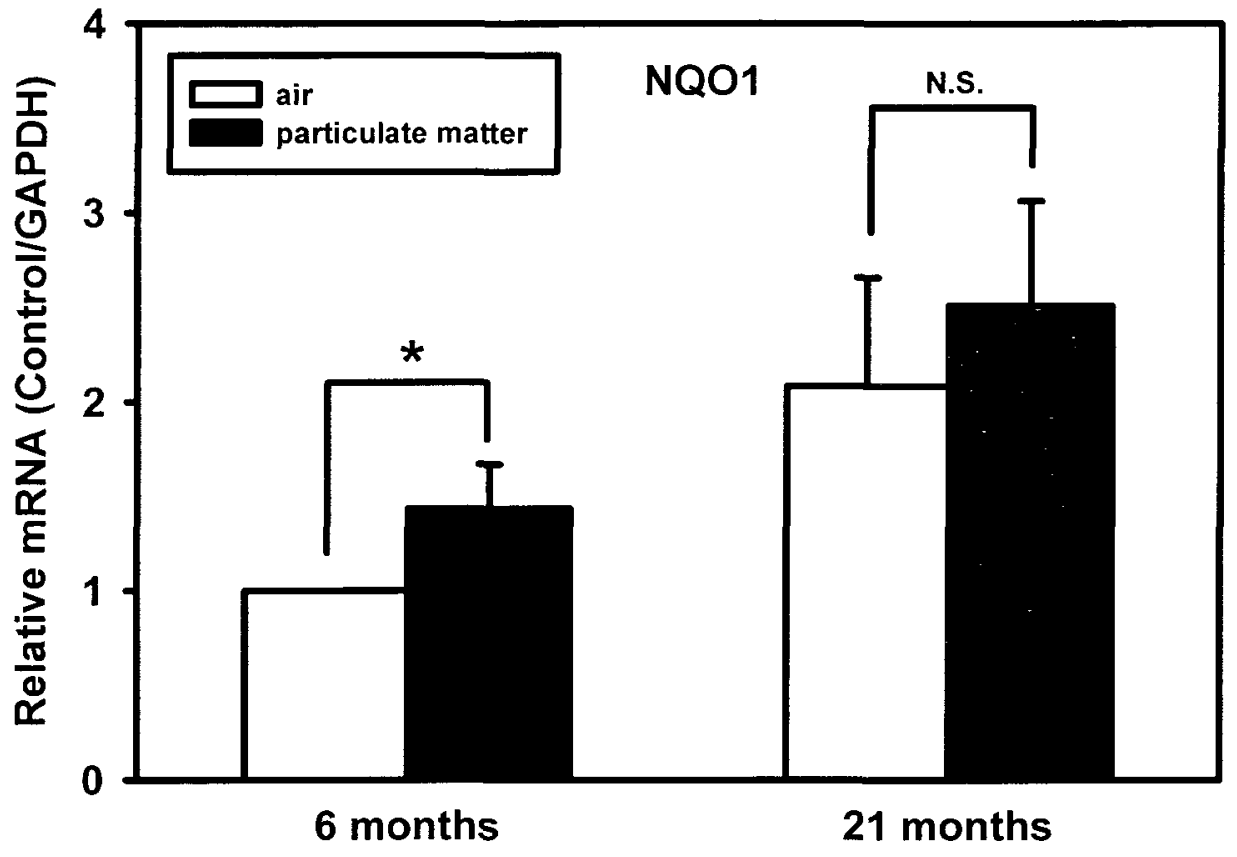

B

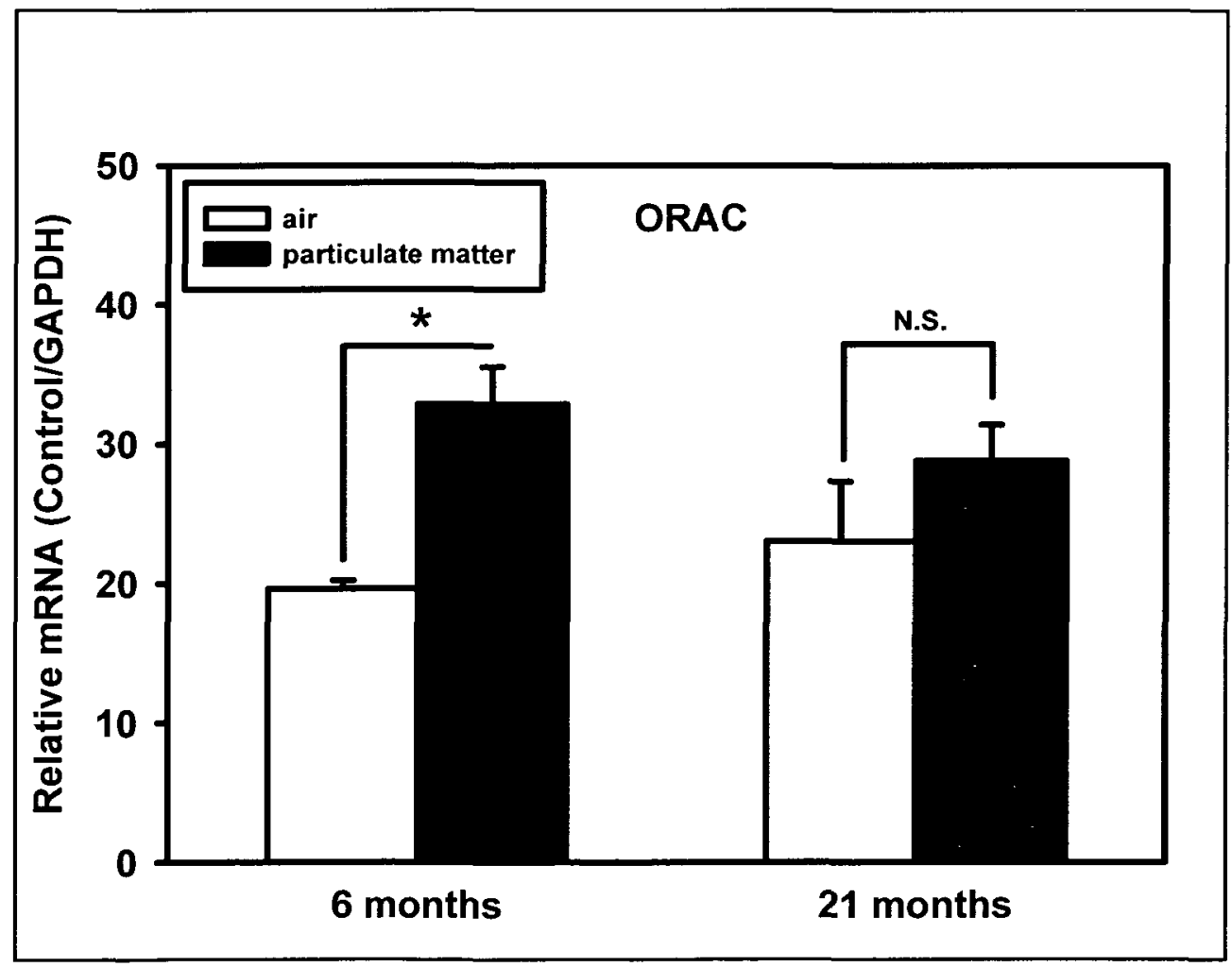




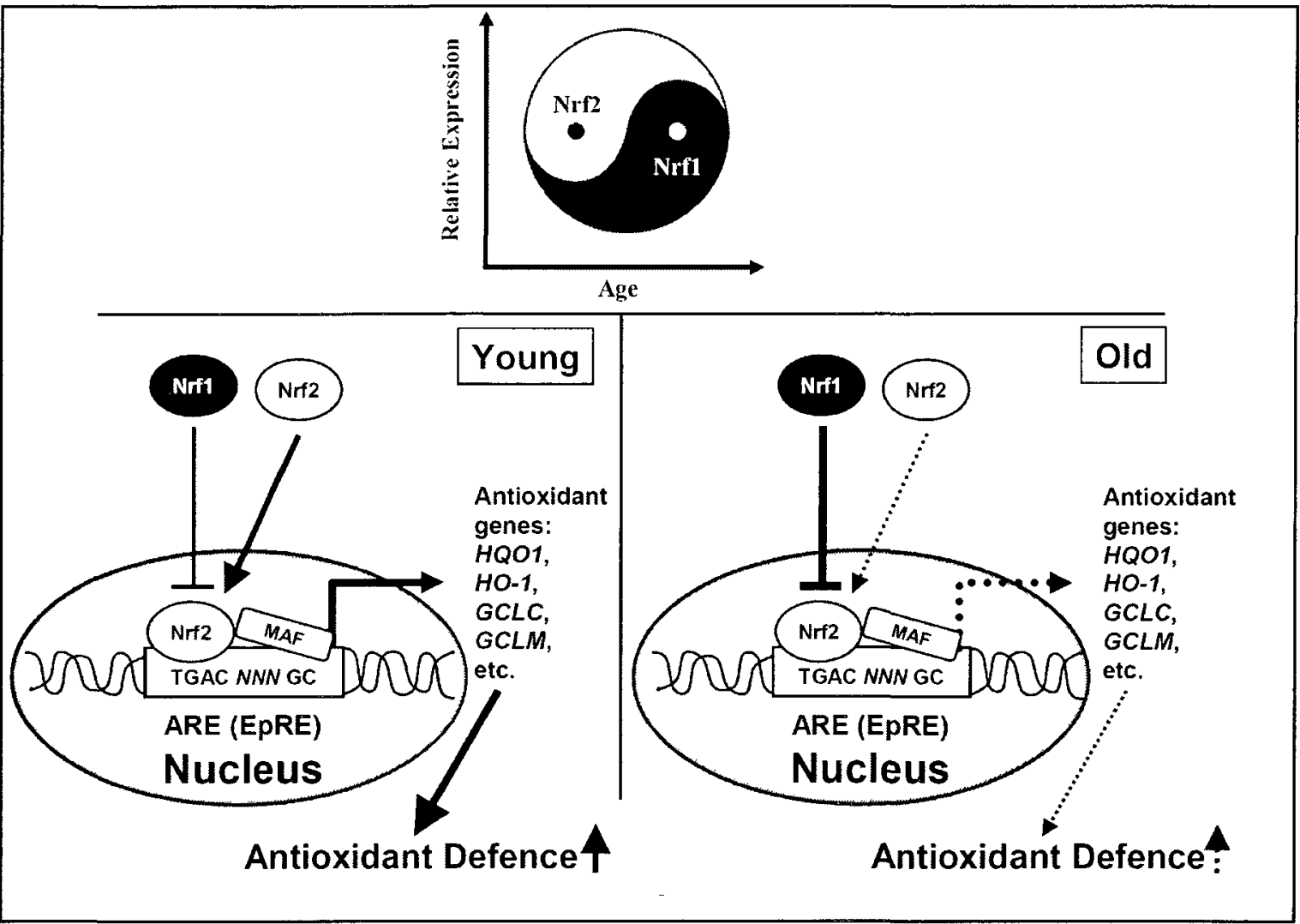

Figure 5.6. The Proposed Model for the Combined Effect of the Particulate Exposure and Aging on the Nrf1/2-ARE Pathway. The ratio of Nrfl (thought to act as an Nrf2/ARE inhibitor) and Nrf2 (the activator of the ARE-driven gene expression) is proposed to be the underlying cause for the reduced responsiveness of the ARE pathway to airborne particle-induced oxidative stress. In young animals, the inhibitory effect of Nrfl on the ARE-driven gene expression, observable with $\mathrm{Nrfl}$ silencing (Figure 5.1A) is not seen clearly due to relatively high Nrf2 content. This situation changes dramatically in old animals, where the Nrf1/Nrf2 ratio is increased as a result of lower Nrf2 and higher Nrfl protein expression. As a result, the inhibitory potential of Nrfl on both basal and oxidative stress- (created by the exposure to the airborne particles) inducible expression of the ARE target genes is more pronounced. See the text for further details. 
6 Chapter: General Discussion 


\subsection{Towards Understanding the Physiological Functions of Nrf1}

Since the beginning of this project in 2006 , a significant breakthrough in understanding the mechanisms involved in the control of Nrfl has been achieved. Nearly $50 \%$ of the articles on Nrfl (17 publications) have been published since 2006, out of 36 publications in total since the initial discovery of Nrf1 in 1993 by the Jefferson Chan group (Chan et al., 1993). Compared to Nrf1, the number of publications on Nrf2 seems to be growing exponentially, totaling nearly 4,000 since $\mathrm{Nrf2}$ discovery in 1994 (Figure 6.1). One can argue that such a discrepancy stems from the fact that Nrf2 global knockout mice are viable, while Nrfl-deficient mice die during embryonic development (Chan et al., 1998).
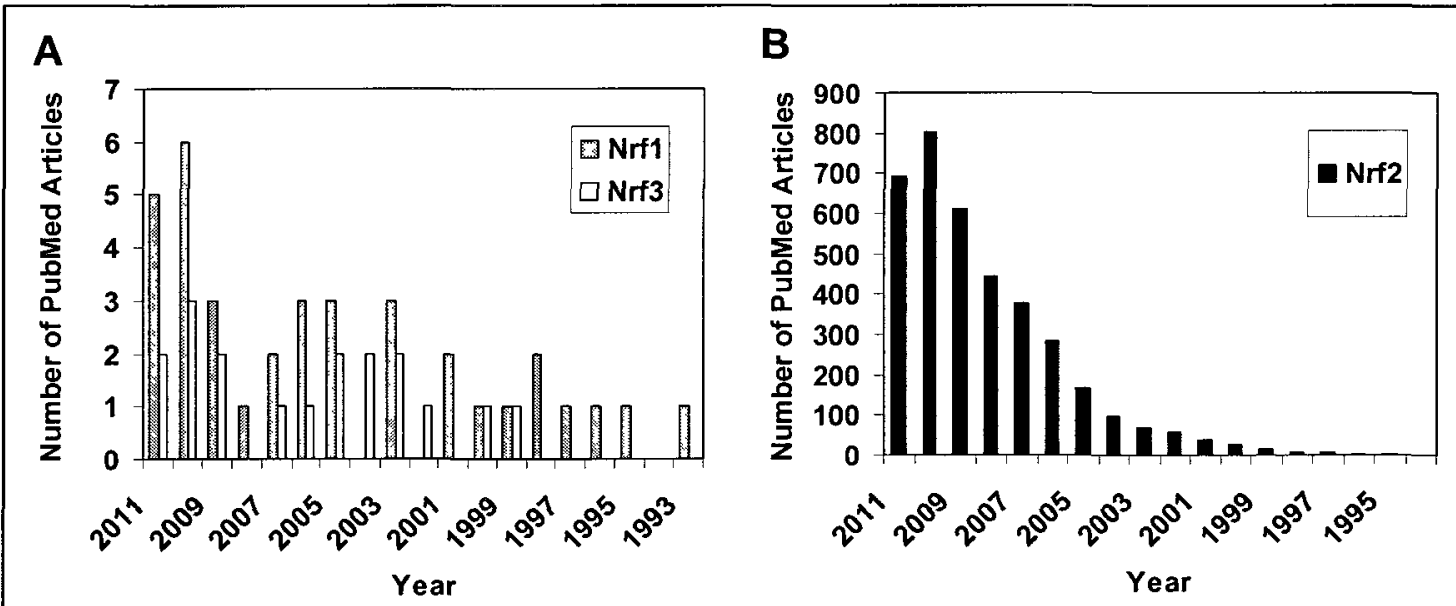

Figure 6.1. The Progress of Our Understanding of the Nrf Factors as Revealed by the Number of PubMed Publications. Review publications were not counted. 
However, this view can be challenged by the rate of progress achieved with understanding the regulation and function of a closely-related CNC-bZIP family member, Nrf3. The Nrf3 factor has received even less attention than Nrfl since its discovery in 1999 and has even been called the "Cinderella" of the CNC-bZIP family (Chevillard and Blank, 2011). Unlike Nrfl, the Nrf3 knock-out mice are viable and show no obvious phenotypic abnormalities (Derjuga et al., 2004). The function of Nrf3 is also more apparent upon certain stimuli such as chemical-induced carcinogenesis (Chevillard et al., 2011). One common feature of Nrfl and Nrf3 is that they are both glycosylated and are localized to the ER through their N-terminal domain (Zhang, Kobayashi et al., 2009; Zhang et al., 2009). This might suggest that the physiological functions of these two factors are related to the maintenance of the ER homeostasis against the ER stress as was proposed by Zhang and co-workers (2009) and Zhang and Hayes (2010). While both Nrf1 and Nrf3 have been reported to act as activators of antioxidant gene expression (Zhang et al., 2009; Chénais et al., 2005), their negative control of the ARE-driven gene expression by Nrf3 (Sankaranarayanan and Jaiswal, 2004) and Nrfl (Wang et al., 2007) has also been reported previously and in this study (Chapter 5). On the other hand, Nrf2 is a well-known master regulator of ARE-inducible gene expression (Pi et al., 2010). Therefore, Nrf1 and Nrf3 may simply add another level of complexity to the Nrf1/2/3ARE pathway regulation and this complexity makes it difficult to investigate the effects of the individual factors alone. One interesting hypothesis regarding the inhibition of Nrf2 function by Nrf1 is that Nrfl function might be necessary to turn off the unrestrained antioxidant gene expression, which would otherwise be pro-carcinogenic (Wang et al., 2007). Regardless of the precise reasons for the poor characterization of 
Nrfl and Nrf3 factors, the disproportionately high focus of the scientific community on $\mathrm{Nrf} 2$ seems puzzling and unjustified as both $\mathrm{Nrfl}$ and $\mathrm{Nrf3}$ may play as an important role in human health and disease as Nrf2 does (Sykiotis and Bohmann, 2010).

Recent studies on the regulation of the gene expression of subunits of the proteasome in human Ea.hy926 cells (Steffen et al., 2010) and mouse embryonic fibroblasts (Radhakrishnan et al., 2010) suggested a role of Nrfl in the maintenance of proteasomal-mediated protein degradation. Nrfl brain-specific knockout in mice led to proteasomal impairment, further confirming the importance of $\mathrm{Nrfl}$ as a translational regulator of proteasomal gene expression (Lee et al., 2011). In addition, the involvement of Nrf1 in ER membrane-dependent biological processes has been proposed by Zhang and Hayes (2010). According to this proposal, the amphipathic regions of Nrfl, once integrated in the ER membrane as $\alpha$-helices, can upset the organization of membrane lipids by restraining the diffusion of neighboring lipids (Zhang and Hayes, 2010). Such a hypothesis has been proposed due to the fact that liver-specific knockouts of $\mathrm{Nrfl}$ in mice resulted in the development of non-alcoholic hepatitis, presumably emanating from the increased overexpression of the cytochrome P450 4A enzymes which catalyze the oxidation of fatty acids, as well as diminished ARE-driven gene expression (Xu et al.,

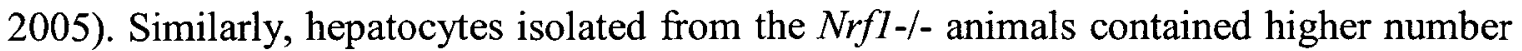
of lipid vesicles and exhibited greater proliferation of smooth ER (Ohtsuji et al., 2008; Xu et al., 2005), which may be yet another indication of the role of Nrfl in the regulation of the ER homeostasis. Other than regulating the antioxidant gene expression, Nrfl also plays a role in: i) inflammation, where Nrfl inhibits the expression of the inducible nitric 
oxide synthase (iNOS) (Berg et al., 2007); ii) bone development, activating the expression of osterix gene (Xing et al., 2007); iii) ondontoblast differentiation, through the regulation of dentin sialophosphoprotein gene expression (Narayanan et al., 2004); and iv) the survival of neurons upon acute brain injury (reviewed in Biswas and Chan, 2010). Taken together, these findings reveal that Nrfl is involved in multiple physiological processes from development to antioxidant response and inflammation, representing exciting subjects for future investigation.

\subsection{Assessment of the Current Models to Study Nrf1 Function}

Unlike the functions of other proteins, such as enzyme-catalyzed reactions where product formation or substrate disappearance can often be easily detected and quantified, studying the function of transcription factors is far more complicated. First, there is no inherent measurable product or property that can be detected in association with a transcription factor's DNA-binding. This limits the in vitro studies of the properties of transcription factors to artificial systems, for example, the one in which a DNA-binding region of a factor is placed upstream of some easily measurable reporter gene, such as luciferase. Such an approach has been used in this project for the study of Nrf1 and Nrf2 functions. However, it suffers from the fact that, in mammalian cells, the transcription of a specific gene can be affected by DNA regions located hundreds or even thousands of base pairs away from the transcription initiation site. As a result, cloning only a short segment of DNA, representing a DNA-binding region of a given factor, does not take into account 
the influence of other regulatory DNA regions. In addition, overexpressing an activator protein in a luciferase type of format can actually decrease rather than increase the reporter activity due to the alteration in the subcellular organization of this factor or corrupting the transcription machinery by exceeding its capacity (Xang et al., 2003). Despite that, such an approach can be an invaluable starting point for future studies in more advanced systems.

To allow for more quantifiable and specific measurement of the DNA-binding function of a given factor, some commercially-available kits exist, such as TransAM systems from Active Motif (http://www.activemotif.com/catalog/180). These rely on the use of specific antibodies, recognizing a DNA-binding protein, bound to a microplate well, coated with a certain DNA segment to which the transcription factor is known to bind. While definitely being more specific than luciferase reporter assays, which can capture the contribution of other transcription factors, this approach, once again, ignores the existence of other regulatory DNA regions outside of the specific sequence recognized and bound by the transcription factor. Another, more practical disadvantage of this technique is its high cost (approximately $\$ 700$ US for a set of 96 reactions) relative to other assays.

In addition, there are electrophoretic mobility shift assays (EMSAs) that allow one to measure the binding of nuclear proteins to a labelled DNA probe. Nuclear extract, isolated from cells, is incubated with the DNA probe, run on a nondenaturing gel and analyzed by autoradiography (an old and hazardous approach) or blotted over to a 
membrane and detected by chemiluminescence (preferred by younger generation of scientists). The weakness of this in vitro technique is that, like luciferase reporter assays, only a short DNA segment is examined, the specificity of the binding must be verified by the addition of specific antibodies, expected to produce a supershift, retarding the DNAprotein complex mobility on a gel. This might be quite a challenge to achieve and often requires special grade of antibodies.

Another, more direct approach is to study the expression of a gene, known to be controlled by a specific transcription factor, with real-time quantitative PCR. A problem with that approach is that a given gene is usually controlled by a battery of transcription factors. An example of this is NQO1, which can be controlled by both Nrf1 and Nrf2, in addition to some other, unknown factors. This problem can be solved by silencing the gene expression of a transcription factor by RNAi, as was performed in Chapter 5, to study the function of Nrfl. Alternatively, a gene, coding for a transcription factor of interest, can be studied by either global or organ-specific knockouts in live animals. Naturally, these animal studies are given more credit and are viewed as the gold standard in transcription factor studies. However, even this approach suffers from some ambiguity, associated with the fact that the effect of removing the factor of interest on the expression of a given gene may be indirect and result as a consequence of some perturbations in the normal cellular homeostasis. For example, Ohtsuji and co-workers (2008) postulated that the upregulation of Nrf2-controlled genes in the livers of $N r f 1$ hepatocyte-specific knockout mice can be due to the fact that the removal of Nrfl may create oxidative stress or other stresses, such that Nrf2 becomes hyperactive to counter such a stress. Figure 6.2 
illustrates how the relative confidence level of transcription factor studies depends upon the experimental design.

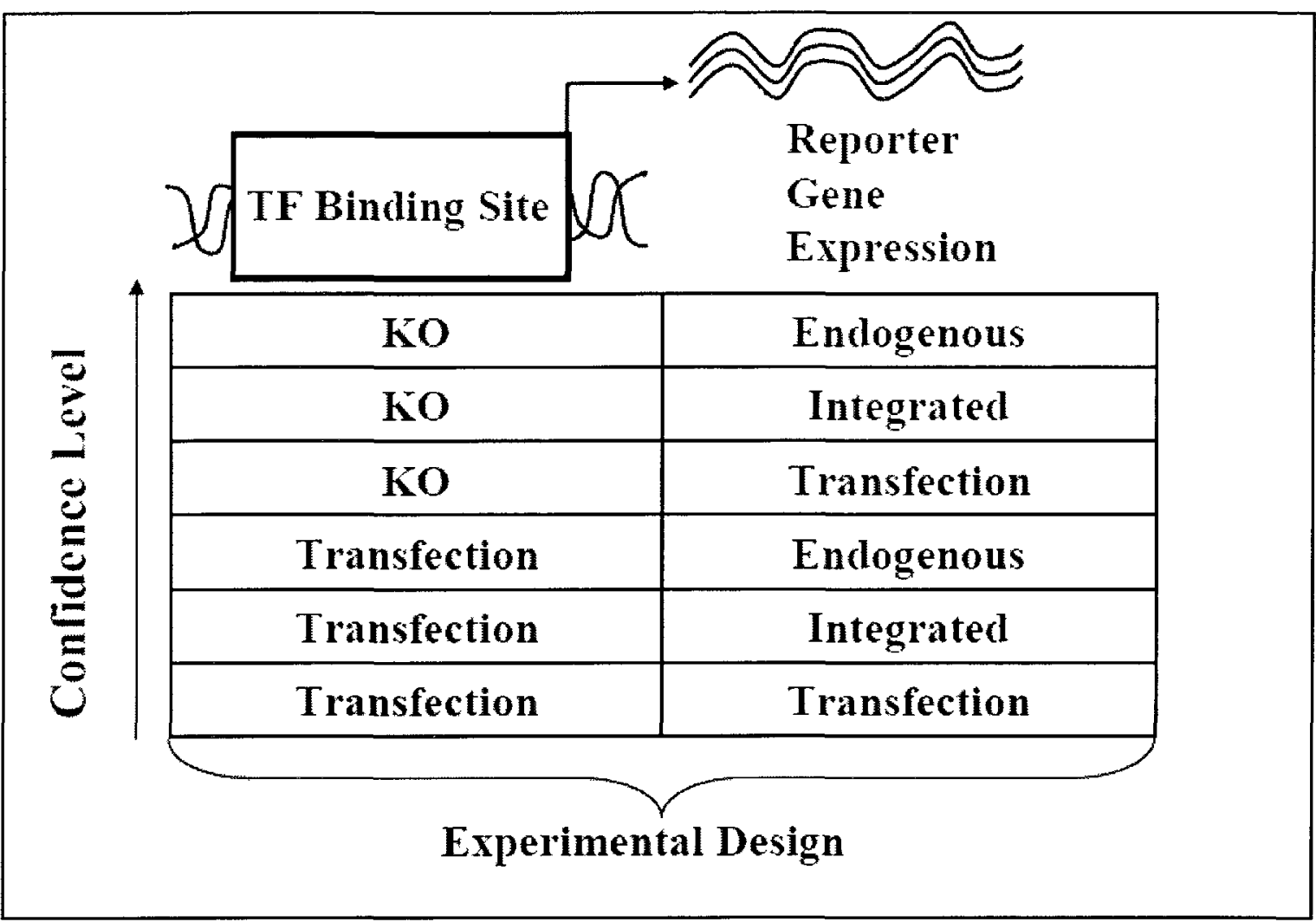

Figure 6.2. The Experimental Design Determines the Confidence in the Results of Transcription Factor Studies. The confidence level of a transcription factor study depends upon the nature of the transcription factor binding site and the type of reporter gene expression. KO refers to knockout studies. The highest confidence is obtained by detecting endogenous gene expression in knockout models. Modified from Xang et al. (2003). 


\subsection{The Potential of Manipulating Nrf1 Activity for Clinical Purposes}

Given the cytoprotective nature of its target genes, the Nrfl/2-ARE pathway is thought by many researchers to be amenable to manipulations aimed at the betterment of the human health. First of all, enhanced detoxification of xenobiotics and potential carcinogens by certain compounds (later known as the Nrf2/ARE inducers) has been described as a viable strategy for chemoprevention using rodent carcinogenesis models (Slocum and Kensler, 2011). To demonstrate that such an enhanced detoxification is afforded by the Nrf2-ARE pathway, $N r f 2$ knockout mice are used in conjunction with carcinogenesis or tumorigenesis models and the efficacy of the inducer is compared in wildtype and knockout animals. For instance, utilizing this technique, it was shown that oltripaz prevents benzo[a]pyrene-induced gastric tumor formation in wildtype, but not in Nrf2-/- animals (Ramos-Gomez et al., 2003). Benzo[a]pyrene contains five benzene rings and readily forms DNA adducts. Nrf2 is thought to activate phase 2 detoxification enzymes, including epoxide hydrolase and NQO1, which, upon the epoxidation reaction, catalyzed by cytochrome p450 enzymes (phase 1 detoxification enzymes), aid in further metabolism and removal of the benzo[a]pyrene metabolites (Slocum and Kensler, 2011).

However, as noted previously, the unrestrained activation of Nrf2 can have a "dark side", namely, pro-carcinogenic effects. This is why Nrfl control could be useful in making sure the Nrf2/ARE pathway activation only occurs when needed and not constitutively. These dark side effects of Nrf2 have been noticed in several cancer types, where the mutations in Nrf2 or Keapl prevent the interactions between the two proteins 
and lead to constitutive Nrf2-ARE activation (reviewed in Slocum and Kensler, 2011). The pro-carcinogenic effect of $\mathrm{Nrf} 2$ overactivation can be due to a) increased capacity of cellular defence enzymes and b) the enhanced ability of malignant cells to detoxify chemotherapeutic drugs by the action of phase 2 detoxification enzymes. Therefore, these undesirable effects of Nrf2 activation must be carefully considered. Even though the Nrf1 niche has been left essentially unoccupied by scientists and clinical researchers, examining virtually any understudied aspect of Nrfl is expected to generate a great deal of attention. One very interesting question that could be considered is whether one can activate $\mathrm{Nrfl}$ in the instances of cancers with increased overexpression of Nrf2. Can such interference counteract the pro-carcinogenic effects of excessive Nrf2 activity? This would require the design of a Nrfl inducer and a good starting point would be to consider inducing Nrfl by disrupting its association with the ER.

Despite some potentially undesirable effects of the increased Nrf2 nuclear presence, there is some suggestion that reversing or preventing the age-associated decline of Nrf2 function can be beneficial. For example, Noyan-Ashraf and co-workers (2008) reported that adding a known phase 2 detoxification enzyme inducer, 2(3)-tert-butyl-4hydroxyanisole (tBHA) into the diet of mice reduced oxidative stress and age-associated weight gain as well as improved the locomotor function. This might suggest that adding certain Nrf2 inducers to our diets can help us achieve healthier aging. Such a view becomes quite popular even among the general public. However, more studies carefully examining the dark side of Nrf2 activation are required. Apparently, a good balance 
between too much and too little Nrf2 has to be found in any attempt to use Nrf2 inducers to human health benefits.

While the benefits of the long-term activation of $\mathrm{Nrf} 2$ are currently understudied and the risks associated with the overexpression of Nrf2 have not been fully assessed, the activation of Nrf2 over a short period of time is expected to hold much promise in the treatment of acute diseases such as brain injury, acute lung injury, cerebral ischemia and intracerebral hemorrhage. This is evidenced using rodent models, where the benefits of Nrf2 activation, administered even after the onset of the tissue damage, were seen (reviewed by Sykiotis and Bohnman, 2010). Given the plethora of the clinical conditions where the activation of Nrf2 can be useful and given the inhibitory effect of Nrfl on the Nrf2/ARE-controlled basal and inducible gene expression, a valid question would be whether Nrfl inactivation can be of any benefit as well? Again, the attempts at keeping Nrfl inert in the ER-bound, glycosylation state could provide a good starting point for these types of studies.

\subsection{Conclusions and Future Directions}

The results showed, for the first time, that $\mathrm{Nrfl}$ is regulated by the proteasome through its continuous, ubiquitin-dependent degradation. This finding, originally reported by the author in 2009 (Chepelev and Willmore, 2009) was later confirmed by Radhakrishnan and others (2010). According to what is now known regarding the proteasomal regulation 
of Nrfl, the repression of its continuous degradation by proteasomal inhibition allows Nrfl to accumulate and activate gene expression of several proteasome genes, regulated through the ARE, including PSMA7 and PSMC1 (Radhakrishnan et al., 2010). This project has also revealed the possibility that $\mathrm{Nrfl}$ is protealytically cleaved by the proteasome at its $\mathrm{N}$-terminus to produce a fragment, migrating as a $23-\mathrm{kDa}$ polypeptide. This conclusion was based on the following: i) the N-terminal location of the Nrf1 region, recognized by the antibody employed; ii) the fact that $\mathrm{p} 23$ signal was diminished following proteasomal inhibition in both COS7 and WFF2002 cells (Figure 3.5), with concomitant increase in the p120 Nrfl iii) the fact that the overexpression of the Nrfl, FLAG-tagged at its N-terminus, could not be seen following FLAG immunoblotting (data not shown), unlike the C-terminally FLAG-tagged Nrfl (Figure 3.7D); iv) the observation that the p23 is observed using two different antibodies, Nrf1 Antibodies H-4 and H-285 (Santa Cruz). Conceptually, this hypothesis makes sense given a similar regulation of NF-kB by the proteasome, in which the inactive p105 is cleaved to the DNA-binding, $\mathrm{p} 50$ form of NF-kB by the 26S proteasome (Magnani et al., 2001; Tanaka et al., 2000). However, more experiments are required to establish that $\mathrm{p} 23$ is indeed a part of Nrfl. These additional experiments could include the use of alternative antibodies, mass spectrometry as well as (or in combination with) epitope tagging of Nrfl at different $\mathrm{N}$-terminal sites to establish the site of fragmentation and to reveal the identity of the p23 fragment. This same list of additional experiments can be extended to reveal the identity of the putative $\mathrm{p} 250$ form of $\mathrm{Nrfl}$, which was seen to be modulated by oxidative stressors and hypoxia. Given that Nrfl is involved in the ARE regulation, it would seem logical to expect some changes at the protein level of Nrfl in response to oxidative stressors, such 
as $\mathrm{AAPH}$ or $\mathrm{tBHQ}$. The fact that $\mathrm{p} 250$ disappeared in response to pro-oxidants may suggest that the inhibitory effect of $\mathrm{Nrfl}$ on $\mathrm{Nrf} 2$ (as discussed extensively in Chapter 5 of this thesis) is somehow repressed in the cell by its removal, such that the Nrf2mediated transcription of the ARE target genes could be activated in response to a stressor; however, more experiments are required, primarily aimed at the identification of $\mathrm{p} 250$, to make any further conclusions. The fact that $\mathrm{p} 250$ is resistant to reducing and denaturing SDS-PAGE conditions suggests that covalent linkage to some other cellular macromolecule may be involved.

In addition to proteasomal regulation, $\mathrm{Nrfl}$ was found to respond positively to the stimulation of intracellular phosphorylation as judged by the luciferease reporter results (Figure 3.8A) while the repression of PKC kinases had the opposite effect. This data suggests that Nrf1 is regulated by phosphorylation, but, it says nothing about the phosphorylation status of Nrf1 itself. Indeed, some other regulatory protein target of phosphorylation may affect Nrfl activity, such that the phosphorylation status of the cell may determine the transactivation activity of Nrfl indirectly. Mass spectrometry analysis might be a useful tool to reveal the identity of phosphorylation sites on Nrfl by using phosphoprotein enrichment through metal-affinity chromatography (Collins et al., 2005). However, this approach might be unable to pinpoint the exact residue(s) undergoing phosphorylation especially among the 25 Ser residues located in the NST region of Nrfl of which 14 Ser residues are located in a row (see Figure 6.3) as the phosphate group can be transferred amongst adjacent phosphorylation sites (J. Smith, personal communication). Similarly, affinity purification (performed in a manner, similar to the 
MLSLKKYLTEGLLQET ILLSLIGVRVDVDTYLTSQLPPLREI ILGPSSAYTQTQEHNLR NTLDGYGIHPKSIDLDNYFTARRLLSQVRALDREQVPTTEVNAWLVHRDPEGSVSGSQP NSGLALESSSGLQDVTGPDNGVRESETEQGFGEDLEDLGAVAPPVSGDLTKEDIDLIDI LWRQDI DLGAGREVFDYSHRQKEQDVEKELRDGGEQDTWAGEGAEALARNLLVDGETGE SFPAQVPSGEDQTALSLEECLRLLEATCPEGENAEFPADISSITEAVPSESEPPALQNN LLSPLLTGTESPFDLEQQWQDLMS I EMQA EVNTSASEILYSAPPGDPLSTNYSLAPN TPINQNVSLHQASLGGCSQDFLLFSPEVESLPVASSSTLLPLAPSNSTSLNSTEGSTNL TGLFFP PQLNGTANDTAGPELPDPLGGLLDEAMLDE ISLMDLA IEEGFNPVQASQLEEE

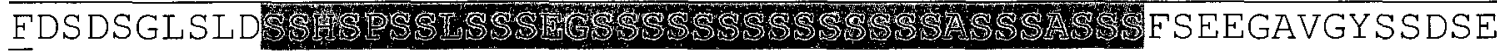
TLDLEEAEGAVGYQPEYSKECRMSYQDPAQLSCLPYLEHVGHNHTYNMAPSALDSADLP PPSALKKGSKEKQADFLDKQM

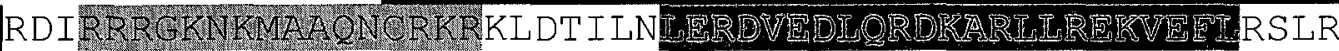
QMKQKVQSLYQEVFGRLRDENGRPYSPSQYALQYAGDGSVLLI PRTMADQQARRQERKP KDRRK

\section{Legend:}

Yellow $=$ LXXLAP Sequence where $\mathrm{X}$ is Any Amino Acid

Light Blue $=$ Poly-Serine Region

Green $=$ Leucine Zipper

Dark Grey $=$ Basic Domain

Light Grey $=$ Asp/Glu-rich (Acidic) Domain

Blue $=$ CNC Domain

Red $=$ Met321 and Met326, the Proposed Start Sites of Internal Translation of Nrf1 Underlined $=$ the Recognition Region (aa. 191-475) of Anti-Nrf1 Antibodies H-4 and H285 (Santa Cruz) and the proposed site for the Nrf1 proteolytic cleavage.

Figure 6.3. The Amino Acid Sequence of Nrf1 Along with Its Domains and Features.

LXXLAP. Shown are some interesting structural features of Nrf1, some of which require future experiments, including the proposed internal translation sites (Met321 and Met326) and putative oxygen-dependent degradation motif (LXXLAP) found in certain proteins such as HIF- $\alpha$, ALAS2 and pyruvate kinase M2. Whether LXXLAP is involved in the $\mathrm{O}_{2}$-dependent regulation of $\mathrm{Nrfl}$ is unknown. The region, recognized by the antibody used in this project and where the proteolytic cleavage of Nrf1 is thought to occur to produce $\mathrm{p} 23$, a $23-\mathrm{kDa}$ fragment of $\mathrm{Nrfl}$, is underlined. The predicted phosphorylation sites are located primarily within the poly-Ser region. 
co-immunoprecipitation shown in Figure 3.3C) could be used in conjunction with mass spectrometry to identify the sites of ubiquitin attachment on Nrfl. In the search for the sites of Nrfl ubiquitination, characteristic shifts of predicted peptide masses of +114.1 and/or +383.2 atomic mass units are expected to occur at the Lys residues, where ubiquitin is attached by the ubiquitin ligases (Denis et al., 2007).

The hypoxic induction of Nrfl-FLAG (Figure 3.7A) was found to correlate well with the decreased expression of the inhibitory p65 form of Nrfl (Figure 3.7E and F). While the documented relationship allows us to hypothesize that the removal of p65 is the underlying cause of the hypoxic activation of $\mathrm{Nrfl}$, more data is required to substantiate this claim. The p65 form has been proposed to arise either through proteolysis or the translation of Nrfl (Chan et al., 1993; See Figure 6.3), but the exact mechanism of p65 generation has not been elucidated yet. To test the hypothesis that hypoxic activation of Nrfl is due to the destabilization of p65, site-directed mutagenesis to abolish the internal translation Met codons can be used and the response of wild-type and mutant Nrfl can be compared. This will be a useful approach only if p65 is, indeed, generated by the internal translation, which should be seen by immunoblotting as the disappearance of the p65 form. If the p65 form of Nrf1 arises due to internal cleavage sites, another approach should be used to disentangle the exact mechanisms by which p65 is generated. For example, mutating different portions of Nrfl near the putative cleavage site, in combination with immunoblotting, may reveal the regions of Nrfl, if not specific residues, where proteolytic processing takes place. 
The activation of Nrf1 and Nrf2 (Figure 4.4) and the induction of the ARE-target genes $\mathrm{NqOl}$ and $\mathrm{HO}-1$ (Table 4.1) by BPA can be also studied in greater detail to gain a better mechanistic insight into the phenomenon. The transcriptional (Table 4.1) and posttranslational modifications of the factors observed (Figure 4.5) provide a wide variety of possible mechanisms that can be addressed. Very little is currently known about the transcriptional regulation of $\mathrm{Nrf1}$ and $\mathrm{Nrf} 2$ genes, other than that, under certain circumstances, the gene expression of these two factors is regulated in the opposite fashion as in the case of whole mouse treatment with butylated hydroxytoluene, a known antioxidant and food additive (Chevillard et al., 2010). Investigating the exact mechanisms by which BPA affects the Nrfl/2-ARE pathway is apparently a more ambitious task than elucidating the types of post-translational modifications of Nrf1, but a global approach using gene microarrays, aimed at identification of other genes affected by BPA, is currently being undertaken at Health Canada and may provide some meaningful insights into the mechanisms of BPA action. What is currently known, using this data, is that a number of the ARE-controlled genes are affected by high concentrations of BPA only. Other pathways affected include a) linoleic acid metabolism, b) glycine, serine and threonine metabolism, and c) cell cycle control of chromosomal replication (Chen et al, in preparation). In addition, microarray analysis of gene expression in human lung fetal fibroblasts revealed that the gene expression of ubiquitinconjugating enzyme UBE2T is downregulated almost 2-fold in response to $100 \mu \mathrm{M} \mathrm{BPA}$ (Chen et al., in preparation). The downregulation of the ubiquitin-proteasomal pathway can stabilize both $\mathrm{Nrf1}$ and $\mathrm{Nrf2}$ in response to BPA and this is a very attractive 
hypothesis that can be tested by examining the ubiquitination state of Nrf1/2 with the help of affinity chromatography and mass spectrometry, as suggested above.

The formation of putative HO-1 dimer is also a very intriguing result, which holds a lot of potential. Since the putative dimer appears to be stable under reducing and denaturing SDS-PAGE conditions, attempts can be made to digest it in gel with trypsin and the generated peptides can be subjected to mass spectrometry analysis. The use of alternative antibodies should be employed, as well, to confirm the identity of the dimer. In addition, the assay of HO-1 activity in which the production of biliverdin and bilirubin from heme under the action of heme oxygenases, can be applied to BPA-treated samples as described by Ryter and colleagues (1998) in order to test the functional significance of HO-1 dimerization. It is expected that dimerization would enhance the HO-1 catalytic activity as suggested by Hwang and others (2009).

Finally, the competition between Nrf1 and Nrf2 was studied. The inhibitory potential of Nrfl on the Nrf2-driven gene expression was seen under the conditions of Nrfl silencing by RNAi and in the luciferase reporter format (Figures 5.1 and 5.2). To gain a better confidence in these important and somewhat counter-intuitive results (which have been supported only by two previously-published reports as discussed in Chapter 5), it is desirable that the effect of $\mathrm{Nrfl}$ silencing be confirmed with another set of RNAi oligos. 
The data described in this thesis also showed that not only Nrf2, but also Nrfl contribute to the ARE binding in young animals and that age is associated with the loss of the ARE-protein binding (Figure 5.3). Interestingly, the exposure of animals with airborne particles abolished age-dependent loss in the ARE binding, which is reminiscent of the concept that ROS are involved in the intracellular signalling pathways and the concept of mitohormesis can be invoked, according to which, the stimulation of mitochondrial ROS generation to a certain extent is believed to have pro-longevity effects (Ristow and Schmeisser, 2011). There is a large body of evidence, suggesting that the stabilization of $\mathrm{Nrf} 2$ has may extend the life span in organisms (reviewed by Lewis et al., 2010). The stimulatory effect of particle exposure was seen as an increased expression of Nqo1 and increased oxidative radical absorbance capacity (Figure 5.5). Paradoxically, this effect of particles was less pronounced in old animals, perhaps, due to the increased stability of Nrf1 (especially its inhibitory p65 form) and the concomitant decrease in Nrf2 expression (Figure 5.4) and it has been discussed extensively throughout the thesis that p65 is known to an act as the Nrf2-ARE pathway inhibitor. Even though there is some evidence that other proteins, such as Bach1, are also stabilized in the samples of aged animals (Zhang et al., in preparation), the inhibitory effect of Nrfl might be of great interest to the scientific community given the immense attention $\mathrm{Nrf} 2$ has received. Nrfl and Nrf2 appear to have a similar binding affinity to the ARE (Ohtsuji et al., 2008) and the mechanisms by which Nrfl can act as an inhibitor are likely to be due to the differences in the transactivation domains of these two factors (Ohtsuji et al., 2008). Once again, $\mathrm{Nrfl} / 2$-other protein interactions can be studied by mass spectrometry, upon 
affinity purification, to reveal the binding partners, of Nrfs which are expected by be chiefly transcriptional co-activators and co-repressors.

In summary, the studies described here have raised some very interesting questions regarding Nrfl regulation and function. First, what is the identity of the p250 form of Nrfl, which responds to multiple stimuli, including hypoxia, pro-oxidants and aging? This seems to represent a common mechanism of Nrfl responsiveness to various stressors. To solve this mystery, affinity purification followed by mass spectrometry, could be helpful in elucidating the modification(s) of Nrfl or its protein-protein or protein-membrane interactions, responsible for its higher molecular weight. Secondly, the same combination of techniques could be instrumental in answering the following questions. As both Nrf1 and Nrf2 bind to the same ARE sequence, what is the difference between their co-activators and co-repressors that determine the transcriptional specificity of these two factors? Third, what can explain the age-related increases in Nrf1 content in the cell? Again, this might be the consequence of the diminished proteasomal performance due to the age-accentuated overload of the proteasome with oxidatively damaged proteins, such that Nrfl becomes more stable. The increased stability of Nrfl could activate the gene expression of proteasomal subunits and relieve the burden on the proteasome, due to the accumulation of oxidatively-damaged and misfolded proteins whose population is known to increase with age (Grim et al., 2011). To answer this question, one would have to not only show that the activity of the ERAD system, responsible for Nrfl degradation declines with age, but also to demonstrate a cause-effect relationship between the declined function of the ERAD and Nrf1 stability. This is not a 
straightforward task since proteasomal inhibitors will stabilize $\mathrm{Nrfl}$, as shown in this project (Chapter 3). Last, a very ambitious approach would be to design a Nrfl activator, useful in the types of cancer with abnormally high Nrf2 activity or to design a Nrfl inhibitor designed to activate $\mathrm{Nrf} 2$ in acute clinical conditions such as brain injury, acute lung injury or cerebral ischemia.

Now is the exciting time for the research on Nrfl and, especially its interaction with Nrf2. Studies aimed at revealing the molecular details of Nrfl regulation are slowly entering the exponential phase to catch up with the research done on $\mathrm{Nrf2}$. The findings on the dominant role of Nrf1 over Nrf2, described in Chapter 5, might facilitate this trend. Future work will determine how the functions of the CNC-bZIP factors are related to each other and what their combined effects are on ARE-driven gene expression. 


\section{Appendices}

\section{Appendix A}

\section{A.1 List of Publications}

The following publications, manuscripts and conference presentations were produced during this project.

\section{Research Papers}

Chepelev, N., Bennitz, J., Wright, J., Smith, J., and Willmore, W. 2009. Oxidative inactivation of citrate synthase and protection with novel antioxidants. J. Enzyme Inhib. Med. Chem. 24, 1319-31.

Cao, X.-L., Corriveau, J., Popovic, S., Coughlan, M., Chepelev, N., Willmore, W., Schrader, T., and Jin, X. 2010. Background bisphenol A in experimental materials and its implication to low-dose in vitro study. Chemosphere. 81, 817-20.

Chepelev, N., Bennitz, J., Huang, T., McBride, S., and Willmore, W. (2011) The NRF1 CNC-bZIP protein is regulated by the proteasome and activated by hypoxia. Submitted to PLoS ONE on July 20, 2011; submission number: PONE-S-11-17302. 
Chepelev, N., Enikanolaiye, M., Chepelev, L., Chen, Q., Scoggan, K., Coughlan, M., Jin, X., and Willmore, W. (2011) Bisphenol A activates Nrfl/2-antioxidant response element pathway in HEK293A cells. Submitted to Chemical Research in Toxicology on March 31, 2011, submission number: tx-2011-00132j.

\section{Conference Proceedings}

Chepelev, N., Wright, J., and Willmore, W. 2006. Oxidative modification and inactivation of citrate synthase by peroxyl radicals and protective effects of novel antioxidants. Free Radic. Biol. Med. 41(S1), S133. Award-winning poster presentation at the $13^{\text {th }}$ Annual Meeting of the SFRBM, Denver, CO.

Chepelev, N., and Willmore, W. 2009. Regulation of Nrfl levels and ARE binding activity during hypoxia in COS7 cells. Abstract for award-winning oral presentation at the $6^{\text {th }}$ Meeting of the Canadian Oxidative Stress Consortium, Winnipeg, MB.

Chepelev, N., and Willmore, G. 2009. The Nrf1 CNC/bZIP protein is regulated by the proteasome and activated by hypoxia. Free Radic. Biol. Med. 47(S1), S14-S15. Awardwinning abstract for poster presentation, the $16^{\text {th }}$ Annual Meeting of the SFRBM, San Francisco, CA.

Chepelev, N., Enikanolaiye, M., Chen, Q., Scoggan, K., Jin, D., and Willmore, W. 2010. Human Antioxidant Response Element-Nrfl/2 pathway-mediated defence against BPA 
exposure. Free Radic. Biol. Med. 49(S1), S127-S128. Abstract for poster presentation at the $17^{\text {th }}$ Annual Meeting of the SFRBM, Orlando, FL.

\section{Reviews}

Chepelev, N., Chepelev, L., Alamgir, M., and Golshani, A. 2008. Large-scale proteinprotein interaction detection: past, present, and future. Biotechnol. Biotechnol. Equip. 22, 513-29.

Chepelev, N., and Willmore, G. 2011. Regulation of iron pathways in response to hypoxia. Free Radic. Biol. Med. 50, 645-66.

\section{Book Chapter}

Chepelev, L., Chepelev, N., Shadnia, H., Willmore, G., Wright, J., and Dumontier, M. 2009. Chapter 7. Development of Small-Molecule Ligands and Inhibitors (p. 115-147). In: Protein Targeting with Small Molecules. Hyroyuki Osada (edit.). John Willey and Sons, Inc. Hoboken, NJ.

\section{Manuscript in preparation}

Chepelev, N., Zhang, H., Liu, H., Willmore, W., Morgan, T., Finch, C., Davies, K., and Forman, H. The Yin-yang of Nrfl and Nrf2 in aging mouse lung. 


\section{News Releases}

"Carleton PhD Student Invited to Work With World Experts on the Aging Process." Carleton University News Release, March 2, 2011.

http://www1.carleton.ca/newsroom/category/news-releases/page/11/

"He'll try to unravel the Nrf1 riddle." Metro Ottawa, March 3, 2011.

http://www.metronews.ca/ottawa/local/article/792386--he-1l-try-to-unravel-an-nrf1-riddle

"Kanata resident works with world experts on aging." Kanata Kourier, March 16, 2011. http://www.yourottawaregion.com/news/local/article/967553--kanata-residentworks-with-world-experts-on-aging

\section{A.2 Ten Simple Rules for Surviving Graduate School}

This section is written in hope that the experience gained by the author during his graduate work might be helpful for other graduate students.

1. During the first year of your studies, try to write a review article. The subject of the review should be based on or be very close to your proposal. Your supervisor is an expert in his field, but he or she often lacks time, necessary for writing. On the other hand, you have some spare time and, under the guidance of your supervisor, can easily review relevant literature and put that into a written form. You will have to do this for your proposal anyways and writing a review is a good way to put everything together. Importantly, our understanding will be assessed by peer-review process, which will give you a good writing and publishing experience. 
2. Attend conferences! Early on, find out what key scientific conferences, relevant to your field of research are out there. Plan to gather enough data, make a poster and present your findings at such a conference. Besides, such conferences often have some stimulating awards, such as travel awards, to help students.

3. Read the "Ten Simple Rules" series of articles, such as "Ten Simple Rules for Graduate Students", freely available at: http://www.ploscompbiol.org/article/info:doi/10.1371/journal.pcbi.0030229

4. Try to think about where you see yourself in the future. This should help you develop the skills necessary for success.

5. When experiments do not work out well, do not give up. Try to look at your data in slightly different way. Maybe, you see something else, other than what you expected? Read the phdcomics: http://www.phdcomics.com/comics.php

6. Develop yourself as a writer and reviewer. You can help your supervisor review a manuscript, submitted for a publication and assigned to your supervisor for reviewing or critically review your supervisor's grant proposal.

7. Become a member of some graduate society/council and socialize with your peers. This helps you develop overall as a person. Try to balance your research with other activities. This will give you more energy to be a more productive researcher and being more productive will give you satisfaction and so on.

8. Time management is essential. Enjoy your life and try to spend each and every moment of it wisely! Avoid downtime. When experiment A does not work, try to work on experiment B at the same time. 
9. Read a lot of articles. Importantly, if something is unclear or if you have an alternative interpretation to what is given in an article, do not hesitate to contact the corresponding author. I found these contacts stimulating and helping myself think in a slightly different manner, especially if the corresponding author is a top expert in the field.

10. Work on the weekends as often as possible. This is a very quiet and productive time. "When I was younger, I always worked in a laboratory on Saturdays. I used to call these my Saturday specials. Sometimes there was an unexpected discovery that was more important than the original objective." These are the words of Theresa Stadtman, a world-renowned biochemist. 


\section{References}

Acker, T.; Fandrey, J.; Acker, H. The good, the bad and the ugly in oxygen-sensing: ROS, cytochromes and prolyl-hydroxylases. Cardiovasc. Res. 71: 195-207; 2006.

Altamura, S.; Muckenthaler, M. U. Iron toxicity in deseases of aging: Alzheimer's disease, Parkinson's disease and atherosclerosis. J. Alzheimer's Dis. 16: 879-895; 2009.

Alonso-Magdalena, P.; Vieira, E.; Soriano, S.; Menes, L.; Burks, D.; Quesada, I.; Nadal, A. Bisphenol A exposure during pregnancy disrupts glucose homeostasis in mothers and adult male offspring. Environ Health Perspect. 118: 1243-1250; 2010.

Aragonés, J.; Schneider, M.; Van Geyte, K.; Fraisl, P.; Dresselaers, T.; Mazzone, M.;

Dirkx, R.; Zacchigna, S.; Lemieux, H.; Jeoung, N. H.; Lambrechts, D.; Bishop, T.; Lafuste, P.; Diez-Juan, A.; Harten, S. K.; Van Noten, P.; De Bock, K.; Willam, C.; Tjwa, M.; Grosfeld, A.; Navet, R.; Moons, L.; Vandendriessche, T.; Deroose, C.; Wijeyekoon, B.; Nuyts, J.; Jordan, B.; Silasi-Mansat, R.; Lupu, F.; Dewerchin, M.; Pugh, C.; Salmon, P.; Mortelmans, L.; Gallez,.B.; Gorus, F.; Buyse, J.; Sluse, F.; Harris, R. A.; Gnaiger, E.; Hespel, P.; Van Hecke, P.; Schuit, F.; Van Veldhoven, P.; Ratcliffe, P.; Baes, M.; Maxwell, P.; Carmeliet, P. Deficiency or inhibition of oxygen sensor Phd1 induces hypoxia tolerance by reprogramming basal metabolism. Nat. Genet. 40: 170-180; 2008. 
Baird, S. K.; Kurz, T.; Brunk, U. T. Metallothionein protects against oxidative stressinduced lysosomal destabilization. Biochem. J. 394: 275-283; 2006.

Bell, E. L.; Emerling, B. M.; Chandel, N. S. Mitochondrial regulation of oxygen sensing. Mitochondrion 5: 322-332; 2005.

Bell, E. L.; Klimova, T. A.; Eisenbart, J.; Moraes, C. T.; Murphy, M. P.; Budinger, G. R.; Chandel, N. S. The Qo site of the mitochondrial complex III is required for the transduction of hypoxic signaling via reactive oxygen species production. $J$. Cell. Biol. 177: 1029-1036; 2007.

Benachour, N.; Aziz, A. Toxic effects of low doses of Bisphenol-A on human placental cells. Toxicol Appl Pharmacol 241: 322-328; 2009.

Berg, D. T.; Gupta, A.; Richardson, M. A.; O'Brien, L. A.; Calnek, D.; Grinnell, B. W. Negative regulation of inducible nitric-oxide synthase expression mediated through transforming growth factor-beta-dependent modulation of transcription factor TCF11. $J$ Biol Chem. 282: 36837-36844; 2007.

Blom, N.; Gammeltoft, S.; Brunak, S. Sequence and structure-based prediction of eukaryotic protein phosphorylation sites. J Mol Biol 294: 1351-1362; 1999. 
Blom, N.; Sicheritz-Ponten, T.; Gupta, R.; Gammeltoft, S.; Brunak, S. Prediction of posttranslational glycosylation and phosphorylation of proteins from the amino acid sequence. Proteomics 4: 1633-1649; 2004.

Bokov, A.; Chaudhuri, A.; Richardson, A. The role of oxidative damage and stress. Mech. Ageing Dev. 125: 811-826; 2004.

Bonkovsky, H. L.; Lincoln, B.; Healey, J. F.; Ou, L. C.; Sinclair, P. R.; Muller-Eberhard, U. Hepatic heme and drug metabolism in rats with chronic mountain sickness. Am. J. Physiol. 251: G467-G474; 1986.

Breuer, W.; Greenberg, E.; Cabantchik, Z. I. Newly delivered transferrin iron and oxidative cell injury. FEBS Lett. 403: 213-219; 1997.

Brunelle, J. K.; Bell, E. L.; Quesada, N. M.; Vercauteren, K.; Tiranti, V.; Zeviani, M.; Scarpulla, R. C.; Chandel, N. S. Oxygen sensing requires mitochondrial ROS but not oxidative phosphorylation. Cell. Metab. 1: 409-414; 2005.

Bunn, H. F.; Poyton, R. O. Oxygen sensing and molecular adaptation to hypoxia. Physiol. Rev. 76: 839-885; 1996. 
Calafat, A. M.; Kuklenyik, Z.; Reidy, J. A.; Caudill, S. P.; Ekong, J.; Needham, L.L. Urinary concentrations of bisphenol $A$ and 4-nonylphenol in a human reference population. Environ. Health Perspect. 113: 391-395; 2005.

Cao, G; Alessio, H. M.; Cutler, R. G. Oxygen-radical absorbance capacity assay for antioxidants. Free Radic Biol Med. 14: 303-311; 1993.

Cao, X-L.; Corriveau, J.; Popovic, S.; Coughlan, M. C.; Chepelev, N.; Willmore, W.; Jin, $\mathrm{X}$. Background bisphenol $\mathrm{A}$ in experimental materials and its implication to low dose in vitro study. Chemosphere 81: 817-820; 2010.

Chachami, G.; Simos, G.; Hatziefthimiou, A.; Bonanou, S.; Molyvdas, P. A.; Paraskeva, E. Cobalt Induces Hypoxia-Inducible Factor-1 Expression in Airway Smooth Muscle Cells by a Reactive Oxygen Species- and PI3K-Dependent Mechanism. Am J Respir Cell Mol Biol. 31: 544-551; 2004.

Chan, J. Y., Han, X. L., Kan, Y. W. Cloning of Nrf1, an NF-E2-related transcription factor, by genetic selection in yeast. Proc Natl. Acad. Sci. USA 90: 11371-11375; 1993.

Chan, J. Y.; Kwong, M.; Lu, R.; Chang, J.; Wang, B.; Yen, T. S.; Kan, Y. W. Targeted disruption of the ubiquitous CNC-bZIP transcription factor, Nrf-1, results in anemia and embryonic lethality in mice. $E M B O J$ 17: 1779-1787; 1998. 
Chan, K.; Kan, Y. W. Nrf2 is essential for protection against acute pulmonary injury in mice. Proc Natl. Acad. Sci. US A 96: 12731-12736; 1999.

Chandel, N. S.; McClintock, D. S.; Feliciano, C. E.; Wood, T. M.; Melendez, J. A.; Rodriguez, A. M.; Schumacker, P. T. Reactive oxygen species generated at mitochondrial complex III stabilize hypoxia-inducible factor-1 alpha during hypoxia: a mechanism of $\mathrm{O}_{2}$ sensing. J. Biol. Chem. 275: 25130-25138; 2000.

Chen, Q.; Gruber, H.; Swist, E.; Pakenham, C.; Ratnayake, W. M.; Scoggan, K. A. Influence of dietary phytosterols and phytostanols on diastolic blood pressure and the expression of blood pressure regulatory genes in SHRSP and WKY inbred rats. Br. J. Nutr. 102: 93-101; 2009.

Chénais, B.; Derjuga, A.; Massrieh, W.; Red-Horse, K.; Bellingard, V.; Fisher, S. J.; Blank, V. Functional and placental expression analysis of the human NRF3 transcription factor. Mol Endocrinol. 19: 125-137; 2005.

Chepelev, N. L.; Bennitz, J. D.; Wright, J. S.; Smith, J. C.; Willmore, W. G. Oxidative modification of citrate synthase by peroxyl radicals and protection with novel antioxidants. J. Enzyme Inhib. Med. Chem. 24: 1319-1331; 2009.

Chepelev, N.; Willmore, G. Regulation of iron pathways in response to hypoxia. Free Radic. Biol. Med. 50: 645-666; 2011. 
Chepelev, N.; Willmore, G. The Nrf1 CNC/bZIP protein is regulated by the proteasome and activated by hypoxia. Free Radic. Biol. Med. 47: S14-S15; 2009 (Abstract).

Chevillard, G.; Blank, V. NFE2L3 (NRF3): the Cinderella of the Cap'n'Collar transcription factors.Cell Mol Life Sci. (2011) DOI: 10.1007/s00018-011-0747-x

Chevillard, G.; Nouhi, Z., Derjuga, A.; Paquet, M.; Blank, V. Nrf3-deficient mice are not protected against acute lung and adipose tissue damages induced by butylated hydroxytoluene. FEBS Lett. 584: 923-928; 2010.

Chevillard, G.; Paquet, M.; Blank, V. Nfe213 (Nrf3) deficiency predisposes mice to T-cell lymphoblastic lymphoma. Blood. 117: 2005-2008; 2011.

Chevion, M.; Berenshtein, E.; Stadtman, E. R. Human studies related to protein oxidation: protein carbonyl content as a marker of damage. Free Radic. Res. 33 Suppl: S99-S108; 2000.

Chitra, K. C.; Latchoumycandane, C.; Mathur, P. P. Induction of oxidative stress by bisphenol A in the epididymal sperm of rats. Toxicology 185: 119-127; 2003.

Collins, M. O.; Yu, L.; Husi, H.; Blackstock, W. P.; Choudhary, J. S.; Grant, S. G. N. Robust enrichment of phosphorylated species in complex mixtures by sequential protein 
and peptide metal-affinity chromatography and analysis by tandem mass spectrometry. Sci. STKE 298: pl6; 2005.

Crawford, L. J.; Walker, B.; Irvine, A. E. Proteasome inhibitors in cancer therapy. J Cell Commun Signal. 5:101-110; 2011.

Czibik, G.; Sagave, J.; Martinov, V.; Ishaq, B.; Sohl, M.; Sefland, I.; Carlsen, H.; Farnebo, F.; Blomhoff, R.; Valen, G. Cardioprotection by hypoxia-inducible factor 1 alpha transfection in skeletal muscle is dependent on haem oxygenase activity in mice. Cardiovasc. Res. 82: 107-114; 2009.

Denis, N. J.; Vasilescu, J.; Lambert, J. P.; Smith, J. C.; Figeys, D. Tryptic digestion of ubiquitin standards reveals an improved strategy for identifying ubiquitinated proteins by mass spectrometry. Proteomics 7: 868-874; 2007.

Derjuga, A.; Gourley, T. S.; Holm, T. M.; Heng, H. H.; Shivdasani, R. A.; Ahmed, R.; Andrews, N. C.; Blank, V. Complexity of CNC transcription factors as revealed by gene targeting of the Nrf3 locus. Mol Cell Biol. 24: 3286-3294; 2004.

Dhakshinamoorthy, S.; Jain, A. K.; Bloom, D. A.; Jaiswal, A. K. Bach1 competes with Nrf2 leading to negative regulation of the antioxidant response element (ARE)-mediated $\mathrm{NAD}(\mathrm{P}) \mathrm{H}$ :quinone oxidoreductase 1 gene expression and induction in response to antioxidants. J. Biol. Chem. 280: 16891-16900; 2005. 
Diabaté, S.; Bergfeldt, B.; Plaumann, D.; Ubel, C.; Weiss, C. Anti-oxidative and inflammatory responses induced by fly ash particles and carbon black in lung epithelial cells. Anal Bioanal Chem. (2011) DOI: 10.1007/s00216-011-5102-4

Dickinson, D. A.; Iles, K. E.; Zhang, H.; Blank, V.; Forman, H. J. Curcumin alters EpRE and AP-1 binding complexes and elevates glutamate-cysteine ligase gene expression. FASEB J 17: 473-475; 2003.

Dinkova-Kostova, A. T.; Talalay, P. NAD(P)H:quinone acceptor oxidoreductase 1 (NQO1), a multifunctional antioxidant enzyme and exceptionally versatile cytoprotector. Arch Biochem Biophys. 501: 116-123; 2010.

Donaldson, K.; Tran, C. L. Inflammation caused by particles and fibers. Inhalation Toxicol. 14: 5-27; 2002.

Eustice, D. C.; Feldman, P. A.; Colberg-Poley, A. M.; Buckery, R. M.; Neubauer, R. H. A sensitive method for the detection of beta-galactosidase in transfected mammalian cells. Biotechniques 11: 739-740, 742-743; 1991.

Eyssen-Hernandez, R.; Ladoux, A. Frelin, C. Differential regulation of cardiac heme oxygenase-1 and vascular endothelial growth factor mRNA expressions by hemin, heavy metals, heat shock and anoxia. FEBS Lett. 382: 229-233; 1996. 
Fähling, M. Cellular oxygen sensing, signalling and how to survive translational arrest in hypoxia. Acta Physiol. 195: 205-230; 2009.

Furukawa, M.; Xiong, Y. BTB protein Keap1 targets antioxidant transcription factor Nrf2 for ubiquitination by the Cullin 3-Roc1 ligase. Mol Cell Biol 25: 162-171; 2005.

Galaris, D.; Pantopoulos, K. Oxidative stress and iron homeostasis: mechanistic and health aspects. Crit. Rev. Clin. Lab. Sci. 45:1-23; 2008.

Ginsberg, G.; Hattis, D. ; Russ, A.; Sonawane, B. Pharmacokinetic and pharmacodynamic factors that can affect sensitivity to neurotoxic sequelae in elderly individuals. Environ Health Perspect.113: 1243-1249; 2005.

Goldberg, M. A.; Dunning, S. P.; Bunn, H. F. Regulation of the erythropoietin gene: evidence that the oxygen sensor is a heme protein. Science 242: 1412-1415; 1988.

Gong, P.; Hu, B.; Stewart, D.; Ellerbe, M.; Figueroa, Y.G.; Blank, V.; Beckman, B. S.; Alam, J. Cobalt induces heme oxygenase-1 expression by a hypoxia-inducible factorindependent mechanism in Chinese hamster ovary cells: regulation by Nrf2 and MafG transcription factors. J. Biol. Chem. 276: 27018-27025; 2001. 
Gordon, C. J.; Gottipolu, R. R.; Kenyon, E. M.; Thomas, R.; Schladweiler, M. C.; Mack, C. M.; Shannahan, J. H.; Wallenborn, J. G.; Nyska, A.; MacPhail, R. C.; Richards, J. E.; Devito, M.; Kodavanti, U. P. Aging and susceptibility to toluene in rats: a pharmacokinetic, biomarker, and physiological approach. $J$ Toxicol Environ Health A. 73: $301-318 ; 2010$.

Grimm, S.; Hoehn, A.; Davies, K. J.; Grune, T. Protein oxidative modifications in the ageing brain: consequence for the onset of neurodegenerative disease. Free Radic Res. 45: 73-88; 2011.

Guzy, R. D.; Hoyos, B.; Robin, E.; Chen, H.; Liu, L.; Mansfield, K. D.; Celeste Simon, M.; Hammerling, U.; Schumacker, P. T. Mitochondrial complex III is required for hypoxia-induced ROS production and cellular oxygen sensing. Cell. Metab. 1: 401-408; 2005.

Guzy, R. D.; Mack, M. M.; Schumacker, P. T. Mitochondrial complex III is required for hypoxia-induced ROS production and gene transcription in yeast. Antioxid. Redox Signal. 9: $1317-1328 ; 2007$.

Halliwell, B. The wanderings of a free radical. Free Radic. Biol. Med. 46: 531-542; 2009. Halliwell, B.; Gutteridge, J. M. C. Free radicals in biology and medicine. Fourth Edition. New York: Oxford University Press Inc.; 2007. 
Hänze, J.; Eul, B. G.; Savai, R.; Krick, S.; Goyal, P.; Grimminger, F.; Seeger, W.; Rose, F. RNA interference for HIF-1alpha inhibits its downstream signalling and affects cellular proliferation. Biochem. Biophys. Res. Commun. 312: 571-577; 2003.

Harman, D. Aging: a theory based on free radical and radiation chemistry. J. Gerontol. 11: $298-300 ; 1956$.

Ho, H.K.; Jia, Y.; Coe, K. J.; Gao, Q.; Doneanu, C. E.; Hu, Z.; Bammler, T. K.; Beyer, R. P.; Fausto, N.; Bruschi, S. A.; Nelson, S. D. Cytosolic heat shock proteins and heme oxygenase-1 are preferentially induced in response to specific and localized intramitochondrial damage by tetrafluoroethylcysteine. Biochem Pharmacol. 72: 80-90; 2006.

Huang, L. E.; Willmore, W. G.; Gu, J.; Goldberg, M. A.; Bunn, H. F. Inhibition of hypoxia-inducible factor 1 activation by carbon monoxide and nitric oxide. Implications for oxygen sensing and signaling. J. Biol. Chem. 274: 9038-9044; 1999.

Hussain, H. H.; Babic, G.; Durst, T.; Wright, J. S.; Flueraru, M.; Chichirau, A.; Chepelev, L. L. Development of novel antioxidants: design, synthesis, and reactivity. J. Org. Chem. 68: 7023-7032; 2003. 
Hwang, H. W.; Lee, J. R.; Chou, K. Y.; Suen, C. S.; Hwang, M. J.; Chen, C.; Shieh, R. C.; Chau, L.Y. Oligomerization is crucial for the stability and function of heme oxygenase-1 in the endoplasmic reticulum. J. Biol. Chem. 284: 22672-22679; 2009.

Igarashi, K.; Hoshino, H.; Muto, A.; Suwabe, N.; Nishikawa, S.; Nakauchi, H.; Yamamoto, M. Multivalent DNA binding complex generated by small Maf and Bach1 as a possible biochemical basis for beta-globin locus control region complex. J. Biol. Chem. 273: $11783-11790 ; 1998$.

Igarashi, K.; Kataoka, K.; Itoh, K.; Hayashi, N.; Nishizawa, M.; Yamamoto, M. Regulation of transcription by dimerization of erythroid factor NF-E2 p45 with small Maf proteins. Nature 367: 568-72; 1994.

Igarashi, K.; Sun, J. The heme-Bach1 pathway in the regulation of oxidative stress response and erythroid differentiation. Antioxid. Redox Signal. 8: 107-118; 2006.

Imhoff, B. R.; Hansen, J. M. Extracellular redox status regulates Nrf2 activation through mitochondrial reactive oxygen species. Biochem J 424: 491-500; 2009.

Iso, T.; Watanabe, T.; Iwamoto, T.; Shimamoto, A.; Furuichi, Y. DNA damage caused by bisphenol A and estradiol through estrogenic activity. Biol. Pharm. Bull. 29: 206-210; 2006. 
Itoh, K.; Wakabayashi, N.; Katoh, Y.; Ishii, T.; Igarashi, K.; Engel, J. D.; Yamamoto, M. Keap1 represses nuclear activation of antioxidant responsive elements by Nrf2 through binding to the amino-terminal Neh2 domain. Genes Dev 1: 76-86; 1999.

Ivan, M.; Kondo, K.; Yang, H.; Kim, W.; Valiando, J.; Ohh, M.; Salic, A.; Asara, J. M.; Lane, W. S.; Kaelin, W. G. Jr. HIF $\alpha$ targeted for VHL-mediated destruction by proline hydroxylation: implications for $\mathrm{O}_{2}$ sensing. Science 292: 464-468; 2001.

Jaakkola, P.; Mole, D. R.; Tian, Y. M.; Wilson, M. I.; Gielbert, J.; Gaskell, S. J.; Kriegsheim, A. V.; Hebestreit, H. F.; Mukherji, M.; Schofield, C. J.; Maxwell, P. H.; Pugh, C. W.; Ratcliffe, P. J. Targeting of HIF- $\alpha$ to the von Hippel-Lindau ubiquitylation complex by $\mathrm{O}_{2}$-regulated prolyl hydroxylation. Science 292: 468-472; 2001.

Jung, K. A.; Kwak, M. K. The Nrf2 system as a potential target for the development of indirect antioxidants. Molecules 10: 7266-7291; 2010.

Kabuto, H.; Hasuike, S.; Minagawa, N.; Shishibori, T. Effects of bisphenol A on the metabolisms of active oxygen species in mouse tissues. Environ. Res. 93: 31-35; 2003.

Kadoma, Y.; Fujisawa, S. Kinetic evaluation of reactivity of bisphenol A derivatives as radical scavengers for methacrylate polymerization. Biomaterials 21: 2125-2130: 2000.

Kaelin, W. G. Jr. ROS: really involved in oxygen sensing. Cell. Metab. 1: 357-358; 2005. 
Kang, J. H.; Kito, K.; Kondo, F. Factors influencing the migration of bisphenol A from cans. J Food Prot 66: 1444-1447; 2003.

Katayose, D.; Isoyama, S.; Fujita, H.; Shibahara, S. Separate regulation of heme oxygenase and heat shock protein $70 \mathrm{mRNA}$ expression in the rat heart by hemodynamic stress. Biochem. Biophys. Res. Commun. 191: 587-594; 1993.

Katoh, Y.; Itoh, K.; Yoshida, E.; Miyagishi, M.; Fukamizu, A.; Yamamoto, M. Two domains of Nrf2 cooperatively bind CBP, a CREB binding protein, and synergistically activate transcription. Genes Cells 6: 857-868; 2001.

Kim, T. K.; Kim, T. H.; Maniatis, T. Efficient recruitment of TFIIB and CBP-RNA polymerase II holoenzyme by an interferon-b enhanceosome in vitro. Proc. Natl. Acad. Sci. USA 95: 12191-12196; 1998.

Kinch, L. N.; Ginalski, K.; Grishin, N. V. Site-2 protease regulated intramembrane proteolysis: Sequence homologs suggest an ancient signaling cascade. Protein Sci. 15: $84-93 ; 2006$.

Kitamuro, T.; Takahashi, K.; Ogawa, K.; Udono-Fujimori, R.; Takeda, K.; Furuyama, K.; Nakayama, M.; Sun, J.; Fujita, H.; Hida, W.; Hattori, T.; Shirato, K.; Igarashi, K.; 
Shibahara, S. Bach1 functions as a hypoxia-inducible repressor for the heme oxygenase-1 gene in human cells. $J$ Biol Chem 278: 9125-9133; 2003.

Klimova, T.; Chandel, N. S. Mitochondrial complex III regulates hypoxic activation of HIF. Cell Death Differ. 15: 660-666; 2008.

Kobayashi, A.; Kang, M. I.; Watai, Y.; Tong, K. I.; Shibata, T.; Uchida, K.; Yamamoto, M. Oxidative and electrophilic stresses activate Nrf2 through inhibition of ubiquitination activity of Keap1. Mol Cell Biol 26: 221-229; 2006.

Kohgo, Y.; Ikuta, K.; Ohtake, T.; Torimoto, Y.; Kato, J. Body iron metabolism and pathophysiology of iron overload. Int. J. Hematol. 88: 7-15; 2008.

Kovacic, P. How safe is bisphenol A? Fundamentals of toxicity: metabolism, electron transfer and oxidative stress. Med. Hypotheses 75: 1-4; 2010.

Kruszewski, M. Labile iron pool: the main determinant of cellular response to oxidative stress. Mut. Res. 531: 81-92; 2003.

Kuznetsova, A. V.; Meller, J.; Schnell, P. O.; Nash, J. A.; Ignacak, M. L.; Sanchez, Y.; Conaway, J. W.; Conaway, R. C.; Czyzyk-Krzeska, M. F. von Hippel-Lindau protein binds hyperphosphorylated large subunit of RNA polymerase II through a proline 
hydroxylation motif and targets it for ubiquitination. Proc. Natl. Acad. Sci. US A 100: 2706-2711; 2003.

Kwak, M. K.; Itoh, K.; Yamamoto, M.; Kensler, T. W. Enhanced expression of the transcription factor Nrf2 by cancer chemopreventive agents: role of antioxidant response element-like sequences in the nrf2 promoter. Mol Cell Biol 9: 2883-2892; 2002.

Kwong, M.; Kan, Y. W.; Chan, J. Y. The CNC basic leucine zipper factor, Nrfl, is essential for cell survival in response to oxidative stress-inducing agents. Role for Nrfl in gamma-gcs(l) and gss expression in mouse fibroblasts. $J$ Biol Chem 274: 37491-37498; 1999.

Larkin, M. A.; Blackshields, G.; Brown, N. P.; Chenna, R.; McGettigan, P. A.; McWilliam, H.; Valentin, F.; Wallace, I. M.; Wilm, A.; Lopez, R.; Thompson, J. D.; Gibson, T. J.; Higgins, D. G. Clustal W and Clustal X version 2.0. Bioinformatics 23: 2947-2948; 2007.

Lang, I. A.; Galloway, T. S.; Scarlett, A.; Henley, W. E.; Depledge, M.; Wallace, R. B.; Melzer, D. Association of urinary bisphenol A concentration with medical disorders and laboratory abnormalities in adults. JAMA 300: 1303-1310; 2008.

Lee, C. S.; Lee, C.; Hu, T; Nguyen, J. M.; Zhang, J.; Martin, M. V.; Vawter, M. P.; Huang, E. J.; Chan, J. Y. Loss of nuclear factor E2-related factor 1 in the brain leads to 
dysregulation of proteasome gene expression and neurodegeneration. Proc Natl. Acad. Sci. US A 108: 8408-8413; 2011.

Lee, P. J.; Jiang, B. H.; Chin, B. Y.; Iyer, N. V.; Alam, J.; Semenza, G. L.; Choi, A. M. K. Hypoxia-inducible factor-1 mediates transcriptional activation of the heme oxygenase1 gene in response to hypoxia. J. Biol. Chem. 272: 5375-5381; 1997.

Levonen, A.L.; Inkala, M.; Heikura, T.; Jauhiainen, S.; Jyrkkänen, H. K.; Kansanen, E.; Määttä, K.; Romppanen, E.; Turunen, P.; Rutanen, J.; Ylä-Herttuala, S. Nrf2 gene transfer induces antioxidant enzymes and suppresses smooth muscle cell growth in vitro and reduces oxidative stress in rabbit aorta in vivo. Arterioscler. Thromb. Vasc. Biol. 27: $741-747 ; 2007$.

Lewis, K. N.; Mele, J.; Hayes, J. D.; Buffenstein, R. Nrf2, a guardian of healthspan and gatekeeper of species longevity. Integr Comp Biol. 50: 829-843; 2010.

Li, J.; Johnson, D.; Calkins, M.; Wright, L.; Svendsen, C.; Johnson, J. Stabilization of Nrf2 by tBHQ confers protection against oxidative stress-induced cell death in human neural stem cells. Toxicol Sci 83: 313-328; 2005.

Li, N.; Sioutas, C.; Cho, A.; Schmitz, D.; Misra, C.; Sempf, J,; Wang, M.; Oberley, T.; Froines, J.; Nel, A. Ultrafine particulate pollutants induce oxidative stress and mitochondrial damage. Environ Health Perspect 111: 455-460; 2003. 
Li, Y. J.; Takizawa, H.; Kawada, T. Role of oxidative stresses induced by diesel exhaust particles in airway inflammation, allergy and asthma: their potential as a target of chemoprevention. Inflamm Allergy Drug Targets. 9: 300-305; 2010.

Lin, P. H.; Chiang, M. T.; Chau, L. Y. Ubiquitin-proteasome system mediates heme oxygenase-1 degradation through endoplasmic reticulum-associated degradation pathway. Biochim Biophys Acta. 1783: 1826-1834; 2008.

Magnani, M.; Crinelli, R.; Bianchi, M.; Antonelli, A. The ubiquitin-dependent proteolytic system and other potential targets for the modulation of nuclear factor-kB (NF-kB). Curr Drug Targets 1: 387-399; 2000.

Maines, M. D. Heme oxygenase: clinical applications and functions, pp. 145-201, CRC Press, Boca Raton, FL.; 1992

Mera, K.; Kawahara, K.; Tada, K.; Kawai, K.; Hashiguchi, T.; Maruyama, I.; Kanekura, T. ER signaling is activated to protect human $\mathrm{HaCaT}$ keratinocytes from ER stress induced by environmental doses of UVB. Biochem Biophys Res Commun 397: 350-354; 2010. 
Misra, C.; Kim, S.; Shen, S.; Sioutas, C. A high flow rate, very low pressure drop impactor for inertial separation of ultrafine from accumulation mode particles. $J$ Aerosol Sci 33: 735-752; 2002.

Mitchell, J. H.; Gardner, P. T.; McPhail, D. B.; Morrice, P. C.; Collins, A. R.; Duthie, G. G. Antioxidant efficacy of phytoestrogens in chemical and biological model systems. Arch. Biochem. Biophys. 360: 142-148; 1998.

Morgan, T. E; Davis, D. A.; Iwata, N.; Tanner, J. A.; Snyder, D.; Ning, Z.; Kam, W.; Hsu, Y. T.; Winkler, J. W.; Chen, J. C.; Petasis, N. A.; Baudry, M.; Sioutas, C.; Finch, C. E. Glutamatergic neurons in rodent models respond to nanoscale particulate urban air pollutants in vivo and in vitro. Environ Health Perspect. 119: 1003-1009; 2011.

Morita, T.; Perrella, M. A.; Lee, M. E.; Kourembanas, S. Smooth muscle cell-derived carbon monoxide is a regulator of vascular cGMP. Proc. Natl. Acad. Sci. USA. 92: 1475$1479 ; 1995$.

Motohashi, H.; O'Connor, T.; Katsuoka, F.; Engel, J. D.; Yamamoto, M. Integration and diversity of the regulatory network composed of Maf and CNC families of transcription factors. Gene 294: 1-12; 2002.

Motohashi, H.; Yamamoto, M. Nrf2-Keap1 defines a physiologically important stress response mechanism. Trends Mol. Med. 10: 549-557; 2004. 
Murphy, B. J.; Laderoute, K. R.; Chin, R. J.; Sutherland, R. M. Metallothionein IIA is upregulated by hypoxia in human A431 squamous carcinoma cells. Cancer Res. 54: 5808$5810 ; 1994$.

Murphy, B. J.; Laderoute, K. R.; Short, S. M.; Sutherland, R. M. The identification of heme oxygenase as a major hypoxic stress protein in Chinese hamster ovary cells. $B r . J$. Cancer 64: 69-73; 1991.

Nakayama, M.; Takahashi, K.; Kitamuro, T.; Yasumoto, K.; Katayose, D.; Shirato, K.; Fujii-Kuriyama, Y.; Shibahara, S. Repression of heme oxygenase-1 by hypoxia in vascular endothelial cells. Biochem. Biophys. Res. Commun. 271: 665-671; 2000.

Narayanan, K.; Ramachandran, A.; Peterson, M. C.; Hao, J.; Kolstø, A. B.; Friedman, A. D.; George, A. The CCAAT enhancer-binding protein (C/EBP)beta and Nrf1 interact to regulate dentin sialophosphoprotein (DSPP) gene expression during odontoblast differentiation. J Biol Chem. 279: 45423-32; 2004.

Naughton, D. P. Hypoxia-induced upregulation of the glycolytic enzyme glucose-6phosphate isomerise perpetuates rheumatoid arthritis. Med. Hypotheses 60: 332-334; 2003. 
Nel, A. Atmosphere. Air pollution-related illness: effects of particles. Science 308: 804$806 ; 2005$.

Nguyen, T.; Nioi, P.; Pickett, C. B. The Nrf2-antioxidant response element signaling pathway and its activation by oxidative stress. J Biol Chem 284: 13291-13295; 2009.

Nguyen, T.; Sherratt, P. J.; Huang, H. C.; Yang, C. S.; Pickett, C. B. Increased protein stability as a mechanism that enhances Nrf2-mediated transcriptional activation of the antioxidant response element. J Biol Chem 278: 4536-4541; 2003.

Ning, Z.; Geller, M. D.; Moore, K. F.; Sheesley, R.; Schauer, J. J.; Sioutas, C. Daily variation in chemical characteristics of urban ultrafine aerosols and inference of their sources. Environ Sci Technol. 41: 6000-6006; 2007.

Nouhi, Z.; Chevillard, G.; Derjuga, A.; Blank, V. Endoplasmic reticulum association and N-linked glycosylation of the human Nrf3 transcription factor. FEBS Lett 581: 5401$5406 ; 2007$.

Numazawa, S.; Ishikawa, M.; Yoshida, A.; Tanaka, S.; Yoshida, T. Atypical protein kinase $\mathrm{C}$ mediates activation of NF-E2-related factor 2 in response to oxidative stress. $\mathrm{Am}$ J Physiol Cell Physiol 285: C334-C342; 2003. 
Oberdörster, G.; Oberdörster, E.; Oberdörster, J. Nanotoxicology: an emerging discipline evolving from studies of ultrafine particles. Environ Health Perspect. 113: 823-839; 2005.

O'Connor, J. C.; Chapin, R. E. Critical evaluation of observed adverse effects of endocrine active substances on reproduction and development, the immune system, and the nervous system. Pure Appl. Chem 75: 2099-2123; 2003.

Oh, P. S.; Lim, K. T. Blocking of intracellular ROS production by phytoglycoprotein (30 $\mathrm{kDa}$ causes anti-proliferation in bisphenol A-stimulated Chang liver cells. J. Appl. Toxicol. 28: 749-758; 2008.

Ohtsuji, M.; Katsuoka, F.; Kobayashi, A.; Aburatani, H.; Hayes, J. D.; Yamamoto, M. $\mathrm{Nrf1}$ and Nrf2 play distinct roles in activation of antioxidant response element-dependent genes. J. Biol. Chem. 283: 33554-33562; 2008.

Ooe, H.; Taira, T.; Iguchi-Ariga, S. M.; Ariga, H. Induction of reactive oxygen species by bisphenol A and abrogation of bisphenol A-induced cell injury by DJ-1. Toxicol. Sci. 88: $114-126 ; 2005$.

Padmanabhan, B.; Tong, K. I.; Ohta, T.; Nakamura, Y.; Scharlock, M.; Ohtsuji, M.; Kang, M. I.; Kobayashi, A.; Yokoyama, S.; Yamamoto, M. Structural basis for defects of 
Keap1 activity provoked by its point mutations in lung cancer. Mol. Cell. 21: 689-700; 2006.

Pajonk, F.; van Ophoven, A.; Weissenberger, C.; McBride, W. H.The proteasome inhibitor MG-132 sensitizes PC-3 prostate cancer cells to ionizing radiation by a DAPK-independent mechanism. BMC Cancer. 5: 76; 2005.

Peyssonnaux, C.; Nizet, V.; Johnson, R. S. Role of the hypoxia inducible factors in iron metabolism. Cell Cycle 7: 28-32; 2008.

Pi, J.; Leung, L.; Xue, P.; Wang, W.; Hou, Y.; Liu, D.; Yehuda-Shnaidman, E.; Lee, C.; Lau, J.; Kurtz, T. W.; Chan, J. Y. Deficiency in the nuclear factor E2-related factor-2 transcription factor results in impaired adipogenesis and protects against diet-induced obesity. J Biol Chem. 285: 9292-9300; 2010.

Rada, P.; Rojo, A. I.; Chowdhry, S.; McMahon, M.; Hayes, J. D.; Cuadrado, A. SCF/ $\beta$ TrCP promotes glycogen synthase kinase 3-dependent degradation of the Nrf2 transcription factor in a Keap1-independent manner. Mol Cell Biol. 31: 1121-1133; 2011.

Radhakrishnan, S. K.; Lee, C. S.; Young, P.; Beskow, A.; Chan, J. Y.; Deshaies, R. J. Transcription factor Nrfl mediates the proteasome recovery pathway after proteasome inhibition in mammalian cells. Mol Cell 38: 17-28; 2010. 
Ramos-Gomez, M.; Dolan, P. M.; Itoh, K.; Yamamoto, M.; Kensler, T. W. Interactive effects of nrf2 genotype and oltipraz on benzo[a]pyrene-DNA adducts and tumor yield in mice. Carcinogenesis 24: 461-467; 2003.

Ristow, M.; Schmeisser, S. Extending life span by increasing oxidative stress. Free Radic Biol Med. 51: 327-336; 2011.

Rogers, S.; Wells, R.; Rechsteiner, M. Amino asid sequences common to rapidly degraded proteins: the PEST hypothesis. Science 234: 364-368; 1986.

Rojek, J. M.; Lee, A. M.; Nguyen, N.; Spiropoulou, C. F.; Kunz, S.Site 1 protease is required for proteolytic processing of the glycoproteins of the South American hemorrhagic fever viruses Junin, Machupo, and Guanarito. J Virol. 82: 6045-6051; 2008.

Rojo, A. I.; de Sagarra, M. R.; Cuadrado, A. GSK-3 $\beta$ down-regulates the transcription factor Nrf2 after oxidant damage: relevance to exposure of neuronal cells to oxidative stress. J. Neurochem. 105: 192-202; 2008.

Rothman, R. J.; Serroni, A.; Farber, J. L. Cellular pool of transient ferric iron, chelatable by deferoxamine and distinct from ferritin, that is involved in oxidative cell injury. Mol. Pharmacol. 42: 703-710; 1992. 
Rubin, B. S. Bisphenol A: An endocrine disruptor with widespread exposure and multiple effects. J Steroid Biochem Mol Biol. 2011, doi:10.1016/j.jsbmb.2011.05.002

Ryan, T. P.; Aust, S. D. The role of iron in oxygen-mediated toxicities. Curr. Rev. Toxicol. 22: 119-141; 1992.

Ryter, S. W.; Choi, A. M. Heme oxygenase-1/carbon monoxide: from metabolism to molecular therapy. Am. J. Respir. Cell Mol. Biol. 41: 251-260; 2009.

Ryter, S.; Kvam, E.; Richman, L.; Hartmann, F.; Tyrrell, R. M. A chromatographic assay for heme oxygenase activity in cultured human cells: application to artificial heme oxygenase overexpression. Free Radic Biol Med. 24: 959-971; 1998.

Salazar, M.; Rojo, A. I.; Velasco, D.; de Sagarra, R. M.; Cuadrado, A. Glycogen synthase kinase-3beta inhibits the xenobiotic and antioxidant cell response by direct phosphorylation and nuclear exclusion of the transcription factor Nrf2.J Biol Chem. 281: $14841-14851 ; 2006$.

Salmon, A. B.; Richardson, A.; Pérez, V. I. Update on the oxidative stress theory of aging: does oxidative stress play a role in aging or healthy aging? Free Radic Biol Med. 48: $642-655 ; 2009$. 
Sankaranarayanan, K.; Jaiswal, A. K. Nrf3 negatively regulates antioxidant-response element-mediated expression and antioxidant induction of $\mathrm{NAD}(\mathrm{P}) \mathrm{H}$ :quinone oxidoreductase1 gene. J Biol Chem. 279: 50810-50817; 2004.

Satoh, T.; Harada, N.; Hosoya, T.; Tohyama, K.; Yamamoto, M.; Itoh, K. Keap1/Nrf2 system regulates neuronal survival as revealed through study of keapl gene-knockout mice. Biochem Biophys Res Commun 380: 298-302; 2009.

Schneider-Poetsch, T.; Ju, J.; Eyler, D. E.; Dang, Y.; Bhat, S.; Merrick, W. C.; Green, R; Shen, B.; Liu, J. O. Inhibition of eukaryotic translation elongation by cycloheximide and lactimidomycin. Nature Chem Biol 6: 209-217; 2010.

Schultz, M. A.; Abdel-Mageed, A. B., Mondal, D. The Nrf1 and Nrf2 balance in oxidative stress regulation and androgen signaling in prostate cancer cells. Cancers 2: $1354-1378 ; 2010$.

Sekhar, K. R.; Yan, X. X.; Freeman, M. L. Nrf2 degradation by the ubiquitin proteasome pathway is inhibited by KIAA0132, the human homolog to INrf2. Oncogene 21: 6829$6834 ; 2002$.

Semenza, G. Hypoxia-inducible factor 1: Regulator of mitochondrial metabolism and mediator of ischemic preconditioning. Biochim. Biophys. Acta 1813: 1263-1268; 2011. 
Semenza, G. L.; Roth, P. H.; Fang, H. M.; Wang, G. L. Transcriptional regulation of genes encoding glycolytic enzymes by hypoxia-inducible factor 1. J. Biol. Chem. 269: $23757-23763 ; 1994$.

Seyfried, N. T.; Xu, P.; Duong, D. M.; Cheng, D.; Hanfelt, J.; Peng, J. Systematic approach for validating the ubiquitinated proteome. Anal Chem. 80: 4161-4169; 2008.

Shibahara, S.; Han, F.; Li, B.; Takeda, K. Hypoxia and heme oxygenases: oxygen sensing and regulation of expression. Antioxid. Redox Signal. 9: 2209-2225; 2007.

Shih, P. H.; Yen, G. C. Differential expressions of antioxidant status in aging rats: the role of transcriptional factor Nrf2 and MAPK signaling pathway. Biogerontology 8:71$80 ; 2007$.

Slocum, S. L.; Kensler, T. W. Nrf2: control of sensitivity to carcinogens. Arch Toxicol. 85: $273-284 ; 2011$.

Soe-Lin, S.; Sheftel, A. D.; Wasyluk, B.; Ponka, P. Nramp1 equips macrophages for efficient iron recycling. Exp. Hematol. 36: 929-937; 2008.

Sohal, R.; Weindruch, R. Oxidative stress, caloric restriction, and aging. Science 273: $59-63 ; 1996$. 
Steffen, J.; Seeger, M.; Koch, A.; Krüger, E. Proteasomal degradation is transcriptionally controlled by TCF11 via an ERAD-dependent feedback loop. Mol Cell 40: 147-158; 2010.

Stewart, D.; Killeen, E.; Naquin, R.; Alam, S.; Alam, J. Degradation of transcription factor Nrf2 via the ubiquitin-proteasome pathway and stabilization by cadmium. J Biol Chem 278: 2396-2402; 2003.

Stiehl, D. P.; Wirthner, R.; Köditz, J.; Spielmann, P.; Camenisch, G.; Wenger, R. H. Increased prolyl 4-hydroxylase domain proteins compensate for decreased oxygen levels. Evidence for an autoregulatory oxygen-sensing system. J. Biol. Chem. 281: 23482$23491 ; 2006$.

Suh, J. H.; Shenvi, S. V.; Dixon, B. M.; Liu, H.; Jaiswal, A. K.; Liu, R. M.; Hagen, T. M. Decline in transcriptional activity of Nrf2 causes age-related loss of glutathione synthesis, which is reversible with lipoic acid. Proc. Natl. Acad. Sci. US A, 101: 3381-3386; 2004.

Sun, J.; Brand, M.; Zenke, Y.; Tashiro, S.; Groudine, M.; Igarashi, K. Heme regulates the dynamic exchange of Bach1 and NF-E2-related factors in the Maf transcription factor network. Proc. Natl. Acad. Sci. USA 101: 1461-1466; 2004. 
Sun, J.; Hoshino, H.; Takaku, K.; Nakajima, O.; Muto, A.; Suzuki, H.; Tashiro, S.; Takahashi, S.; Shibahara, S.; Alam, J.; Taketo, M. M.; Yamamoto, M.; Igarashi, K. Hemoprotein Bach1 regulates enhancer availability of heme oxygenase-1 gene. EMBO J. 21: 5216-5224; 2002.

Sun, L.Y.; Bokov, A.F.; Richardson, A.; Miller, R.A. Hepatic response to oxidative injury in long-lived Ames dwarf mice. FASEB J 25: 398-408; 2011.

Sykiotis, G. P.; Bohmann, D. Stress-activated cap'n'collar transcription factors in aging and human disease. Sci Signal 3: 1-22; 2010.

Taguchi, K.; Motohashi, H.; Yamamoto, M. Molecular mechanisms of the Keap1-Nrf2 pathway in stress response and cancer evolution. Genes Cells. 16:123-140; 2011.

Tanaka, K.; Kawakami, T.; Tateishi, K.; Yashiroda, H.; Chiba, T. Control of IkappaBalpha proteolysis by the ubiquitin-proteasome pathway. Biochimie 83: 351-356; 2001.

Tomobe, K.; Shinozuka, T.; Kuroiwa, M.; Nomura, Y. Age-related changes of Nrf2 and phosphorylated GSK-3 $\beta$ in a mouse model of accelerated aging (SAMP8). Arch Gerontol Geriatr. (2011). DOI:10.1016/j.physletb.2003.10.071 
van Weeren, P. C.; de Bruyu, K. M.; Vries-Smits, A.M .; van Lint, J.; Burgering, B. M., Essential role for protein kinase B $(\mathrm{PKB})$ in insulin-induced glycogen synthase kinase 3 inactivation. J. Biol. Chem. 273: 13150-1315; 1998.

Waleh, N. S.; Calaoagan, J.; Murphy, B. J.; Knapp, A. M.; Sutherland, R. M.; Laderoute, K. R. The redox-sensitive human antioxidant responsive element induces gene expression under low oxygen conditions. Carcinogenesis 19: 1333-1337; 1998.

Wang, G. L. Semenza, G. L. General involvement of hypoxia-inducible factor 1 in transcriptional response to hypoxia. Proc. Natl. Acad. Sci. USA 90: 4304-4308; 1993.

Wang, W.; Chan, J. Y. Nrfl is targeted to the endoplasmic reticulum membrane by an Nterminal transmembrane domain. J Biol Chem 281: 19676-19687; 2006.

Wang, W.; Jaiswal, A. K. Nuclear factor Nrf2 and antioxidant response element regulate NRH:quinone oxidoreductase 2 (NQO2) gene expression and antioxidant induction. Free Radic Biol Med. 40: 1119-1130; 2006.

Wang, W.; Kwok, A. M.; Chan, J. Y. The p65 isoform of Nrfl is a dominant negative inhibitor of ARE-mediated transcription. J Biol Chem 282: 24670-24678; 2007. 
Wright, J. S.; Johnson, E. R.; DiLabio, G. A. Predicting the activity of phenolic antioxidants: Theoretical method, analysis of substituent effects, and application to major families of antioxidants. J. Am. Chem. Soc. 123: 1173-1183; 2003.

Xia, T.; Kovochich, M.; Brant, J.; Hotze, M.; Sempf, J.; Oberley, T.; Sioutas, C.; Yeh, J. I.; Wiesner, M. R.; Nel, A. E. Comparison of the abilities of ambient and manufactured nanoparticles to induce cellular toxicity according to an oxidative stress paradigm. Nano Lett. 6: 1794-1807; 2006.

Xu, Z.; Chen, L.; Leung, L.; Yen, T. S.; Lee, C.; Chan, J. Y. Liver-specific inactivation of the Nrfl gene in adult mouse leads to nonalcoholic steatohepatitis and hepatic neoplasia. Proc Natl. Acad. Sci. U S A 102: 4120-4125; 2005.

Yamasaki, M.; Nomura, T.; Sato, F.; Mimata, H. Metallothionein is up-regulated under hypoxia and promotes the survival of human prostate cancer cells. Oncol. Rep. 18: 1145$1153 ; 2007$.

Yankaskas, J. R.; Haizlip, J. E.; Conrad, M.; Koval, D.; Lazarowski, E.; Paradiso, A. M.;

Rinehart, C. A. J.; Sarkadi, B.; Schlegel, R.; Boucher, R. C. Papilloma virus immortalized tracheal epithelial cells retain a well-differentiated phenotype. Am. J. Physiol. 264: C1219-C1230; 1993. 
Yim, C. Y.; Hibbs, J. B.; McGregor, J. R.; Galinsky, R. E.; Samlowski, W. E. Use of Nacetyl cysteine to increase intracellular glutathione during the induction of antitumor responses by IL-2. J. Immunol. 152: 5796-5805; 1994.

Yoshida, T.; Biro, P.; Cohen, T.; Müller, R. M.; Shibahara, S. Human heme oxygenase cDNA and induction of its mRNA by hemin. Eur. J. Biochem. 171: 457-461; 1988.

Yoshiki, N.; Kubota, T., Aso, T. Identification of heme oxygenase in human endometrium. J Clin Endocrinol Metab. 86: 5033-5038; 2001.

Zhang, X.; Garfinkel M.; Ruden; D. 2003. "Phylogenetic conservation of the Nrf1-Keap1 signalling system," In Signal Transduction by Reactive Oxygen and Nitrogen Species, (Forman H, Fukuto J, Torres M., eds) pp.256-274, Kulwer Academic Publishers, Dordrecht, Netherlands.

Zhang, H.; Forman, H. J. Acrolein induces heme oxygenase-1 through PKC-delta and PI3K in human bronchial epithelial cells. Am J Respir Cell Mol Biol 38: 483-490; 2008.

Zhang, H.; Forman, H. J. Signaling pathways involved in phase II gene induction by alpha, beta-unsaturated aldehydes. Toxicol Ind Health. 25: 269-78; 2009. 
Zhang, Y.; Crouch, D. H.; Yamamoto, M.; Hayes, J. D. Negative regulation of the Nrf1 transcription factor by its N-terminal domain is independent of Keap1: Nrfl, but not Nrf2, is targeted to the endoplasmic reticulum. Biochem J 399: 373-385; 2006.

Zhang, Y.; Hayes, J. D. Identification of topological determinants in the N-terminal domain of transcription factor Nrf1 that control its orientation in the endoplasmic reticulum membrane. Biochem J 430: 497-510; 2010.

Zhang, Y.; Kobayashi, A.; Yamamoto, M.; Hayes, J. D. The Nrf3 transcription factor is a membrane-bound glycoprotein targeted to the endoplasmic reticulum through its $\mathrm{N}$ terminal homology box 1 sequence. J Biol Chem. 284: 3195-210; 2009.

Zhang, Y.; Lucocq, J. M.; Hayes, J. D. The Nrfl CNC/bZIP protein is a nuclear envelope-bound transcription factor that is activated by t-butyl hydroquinone but not by endoplasmic reticulum stressors. Biochem J 418: 293-310; 2009.

Zhang, Y.; Lucocq, J. M.; Yamamoto, M.; Hayes, J. D. The NHB1 (N-terminal homology box 1) sequence in transcription factor Nrfl is required to anchor it to the endoplasmic reticulum and also to enable its asparagine-glycosylation. Biochem $J 408$ : $161-172 ; 2007$.

Zhao, R.; Hou, Y.; Xue, P.; Woods, C. G.; Fu, J.; Feng, B.; Guan, D.; Sun, G.; Chan, J. Y.; Waalkes, M. P.; Andersen, M. E.; Pi, J. Long isoforms of NRF1 contribute to arsenic- 
induced antioxidant response in human keratinocytes. Environ Health Perspect. 119: 56$62 ; 2011$.

Zhu, M.; Fahl, W. E. Functional characterization of transcription regulators that interact with the electrophile response element. Biochem Biophys Res Commun 289: 212-219; 2001.

Zhu, Y.; Zhang, Y.; Ojwang, B. A.; Brantley, M. A. Jr.; Gidday, J. M. Long-term tolerance to retinal ischemia by repetitive hypoxic preconditioning: role of HIF-1 $\alpha$ and heme oxygenase-1. Invest. Ophthalmol. Vis. Sci. 48: 1735-1743; 2007.

\section{$\Omega$}

The fear of the LORD is the beginning of knowledge (Proverbs 1:7) 